University of South Florida

DIGITAL COMMONS

Digital Commons @ University of

@ UNIVERSITY OF SOUTH FLORIDA

South Florida

USF Tampa Graduate Theses and Dissertations

USF Graduate Theses and Dissertations

$11-3-2008$

\title{
Motion-Logger: An Attitude and Motion Sensing System
}

Andres Felipe Marquez

University of South Florida

Follow this and additional works at: https://digitalcommons.usf.edu/etd

Part of the American Studies Commons

\section{Scholar Commons Citation}

Marquez, Andres Felipe, "Motion-Logger: An Attitude and Motion Sensing System" (2008). USF Tampa Graduate Theses and Dissertations.

https://digitalcommons.usf.edu/etd/377

This Thesis is brought to you for free and open access by the USF Graduate Theses and Dissertations at Digital Commons @ University of South Florida. It has been accepted for inclusion in USF Tampa Graduate Theses and Dissertations by an authorized administrator of Digital Commons @ University of South Florida. For more information, please contact digitalcommons@usf.edu. 
Motion-Logger: An Attitude and Motion Sensing System

by

\begin{abstract}
Andres Felipe Marquez
A thesis submitted in partial fulfillment

of the requirements for the degree of

Master of Science in Electrical Engineering

Department of Electrical Engineering

College of Engineering

University of South Florida

Major Professor: Wilfrido A. Moreno, Ph.D.

James T. Leffew, Ph.D.

Mauricio Castillo-Effen, Ph.D.
\end{abstract}

Date of Approval:

November 3, 2008

Keywords: Attitude estimation, Kalman filter, Inertial Measurement Unit (IMU), Accelerometers, Gyroscopes.

(C) Copyright 2008, Andres Felipe Marquez 


\section{Dedication}

To all the people who made it possible for me to write this thesis. 


\section{Acknowledgments}

I thank my major professor Dr. Wilfrido Moreno for his support during this research and the Masters program, which I am successfully finishing at the University of South Florida.

I express my gratefulness to Dr. Mauricio Castillo for his invaluable contributions to the development of the project.

I thank Dr. James Leffew for reading my thesis and providing important feedback. I must also express my gratitude to the personnel of the research center of the James A. Haley VA Hospital, (Tampa, Florida), who provided fundamental support during the planning and execution of the project. Specifically, I received encouragement and support from Dr. Audrey Nelson, Shirley Fitzgerald, Barbara Mckenzie, Shawn Applegarth, Deborah Gavin-Dreschnack, Andrea Spehar and Robert Byrd.

I thank my fellow students Henry Cabra and Allistair Moses for their engagement and technical advice during the development of this research. 


\section{Table of Contents}

List of Tables $\quad$ iii

List of Figures $\quad$ iv

Abstract vii

Chapter 1 Introduction 1

1.1 Motivation 1

1.2 Background 2

1.3 Objectives and Scope of the Research 4

Chapter 2 Related Research 6

2.1 Research Projects $\quad 6$

2.1.1 Wheelchair Usage Monitoring/Logging System 6

2.1.2 Monitoring Seat Feature Usage among Wheelchair Users 9

2.1.3 An Indoor Localization Aid for the Visually Impaired $\quad 12$

$\begin{array}{ll}2.2 \text { Commercially Available Products } & 14\end{array}$

2.2.1 HOBO Pendant G Acceleration Data Logger 14

2.2.2 The "Black Box" - A Powered Wheelchair Usage Monitor 16

$\begin{array}{lll}\text { Chapter } 3 & \text { Systems Engineering } & 18\end{array}$

3.1 Customer Needs, Requirements and Challenges 18

3.2 Functional Analysis $\quad 20$

3.3 Alternative Implementation Solutions $\quad 22$

3.4 Analysis of Alternatives and Comparison $\quad 24$

3.5 Final Design Decisions 25

Chapter 4 Embedded Application Development 30

4.1 Description 30

4.2 Signal Analysis 33

4.3 Sampling Rate $\quad 38$

4.3.1 Fast Motion Experiment $\quad 39$

4.3.2 Slow Motion Experiment 46 
$\begin{array}{lll}\text { Chapter } 5 & \text { Sensor Fusion } & 53\end{array}$

5.1 Accelerometers Based Estimation 53

$\begin{array}{ll}5.2 \text { Kalman Filter } & 54\end{array}$

5.2.1 Kalman Filter Formulation $\quad 55$

$\begin{array}{ll}\text { 5.2.2 Kalman Filter Implementation } & 60\end{array}$

$\begin{array}{lll}\text { Chapter } 6 & \text { Results } & 65\end{array}$

$\begin{array}{ll}6.1 \text { Pilot Study } & 65\end{array}$

$\begin{array}{ll}\text { 6.2 Deployment of Wheelchair Users } & 74\end{array}$

$\begin{array}{lll}\text { Chapter } 7 & \text { Conclusions and Future Work } & 87\end{array}$

$\begin{array}{ll}7.1 \text { Conclusions } & 87\end{array}$

$\begin{array}{ll}7.2 \text { Future Work } & 88\end{array}$

$\begin{array}{ll}\text { References } & 90\end{array}$

$\begin{array}{ll}\text { Appendices } & 92\end{array}$

Appendix A: Tilt Sensor Instructions 93 


\section{List of Tables}

Table 1: Comparison between Referenced Projects 17

Table 2: Angle Values Broken Down in Intervals for Subject \#1 67

Table 3: Angle Values broken Down in Intervals for Subject \#2 69

Table 4: Angle Values Broken Down in Intervals for Subject \#3 70

Table 5: Angle Values Broken Down in Intervals for Subject \#4 72

Table 6: Angle Values Broken Down in Intervals for Subject \#5 73 


\section{List of Figures}

Figure 1: Wheel Rotation Logging Module, [3]

Figure 2: Seating Posture Logging Module, [3] $\quad 8$

Figure 3: Seat Tilt Angle Ranges Accessed by Subjects, [4] 10

Figure 4: Backrest Recline Angle Ranges Accessed by Subjects, [4] 11

Figure 5: White Cane and Hardware, [5] 13

Figure 6: HOBO Pendant Acceleration Data Logger, [6] 15

Figure 7: Different Wheelchair Types for Device Installation $\quad 20$

Figure 8: Proposed Solution Block Diagram 22

Figure 9: Embedded Platform 26

$\begin{array}{ll}\text { Figure 10: Embedded Device } & 27\end{array}$

Figure 11: Velcro Adhered to the Wheelchair and the Data Logger for Coupling 28

Figure 12: Data Logger Attached to a Wheelchair 29

Figure 13: Embedded Application Flow Chart 31

Figure 14: High Pass Digital Filter Response $\quad 34$

Figure 15: Real-Time Signal Processing Algorithm for Selective Data Storage 35

Figure 16: Sample Logged File $\quad 36$

Figure 17: Mechanical Wheelchair used in the Fast Motion Experiment 39 
Figure 18: Estimated Angles for the Fast Motion Experiment

Figure 19: Gyroscopes Spectrum for the Fast Motion Experiment

Figure 20: Spectrum of Accelerometers in the X and Y Directions for the Fast Motion Experiment

Figure 21: Spectrum of Accelerometer in the Z Direction for the Fast Motion Experiment

Figure 22: Estimated Angles after Filtering the Sensors Output

Figure 23: Pitch and Roll Spectrum after Filtering Sensors Output

Figure 24: Electrical Wheelchair used in the Slow Motion Experiment

Figure 25: Estimated Angles for the Slow Motion Experiment

Figure 26: Gyroscopes Spectrum for the Slow Motion Experiment

Figure 27: Spectrum of Accelerometers in the X and Y Directions for the Slow Motion Experiment

Figure 28: Spectrum of Accelerometer in the Z Direction for the Slow Motion Experiment

Figure 29: Estimated Pitch and Roll Spectrum after Filtering

Figure 30: Estimated Angles after Filtering

Figure 31: Gravity Components with the Wheelchair Tilted

Figure 32: Kalman Filter Cycle, [14]

Figure 33: Kalman filter Operation, [14]

Figure 34: Kalman Filter Block Diagram

Figure 35: Results from a 3 and a Half Hour Trial with a Wheelchair User.

Figure 36: Device Attached to a Wheelchair during the Pilot Study 
Figure 38: Estimated Angles for Subject \#2

Figure 39: Estimated Angles for Subject \#3

Figure 40: Estimated Angles for Subject \#4

Figure 41: Estimated Angles for Subject \#5

Figure 42: Experiment for Testing Proper Functionality of the Data-Loggers

Figure 43: Data Logger Attached to a Wheelchair during the Final Deployment Stage

Figure 44: Spreadsheet Displaying the Number of Tips, Falls and Time-Stamps

Figure 45: Pitch and Roll Variations during Tip Detection in Frames 1 and 2 for Subject \#4

Figure 46: Pitch and Roll Variations during Tip Detection in Frames 3 and 4 for Subject \#4

Figure 47: Estimated Angles in Frames 1 and 2 within which Tips were Detected for Subject \#17

Figure 48: Estimated Angles in Frames 3 and 4 within which Tips were Detected for Subject \#17

Figure 49: Pitch and Roll Angles during Frames 1 and 2 within which Tips were Detected for Subject \#8

Figure 50: Pitch and Roll Angles during Frames 3 and 4 within which Tips were Detected for Subject \#8

Figure 51: Pitch and Roll Angles during Frames within which Falls were Detected for Subject \#4

Figure 52: Pitch and Roll during Frames within which Falls were Detected for Subject \#17 


\title{
Motion-Logger: An Attitude and Motion Sensing System
}

\section{Andres Felipe Marquez}

\begin{abstract}
This thesis proposes a motion sensing system for wheelchairs with the main objective of determining tips, falls and risky situations. The system relies on measurements from an Inertial Measurement Unit, (IMU), consisting of a 3-axis accelerometer and a 2-axis gyroscope as the source of information. The IMU was embedded in a portable device, the "Motion Logger", which collects motion data in a Secure Digital memory card after running a real time preprocessing algorithm. The algorithm was designed to reduce energy consumption and memory usage. Actual signal analysis and attitude estimation is carried out offline.

The motion sensing system was developed for determining wheelchair-related falls as part of a major research effort carried out at the research center of the James A Haley VA Hospital Subject Safety Center, Tampa, Florida. The focus of the study concentrated on achieving a thorough understanding of the demographics, nature, consequences and the creation of prediction models for fall events.
\end{abstract}

The main goal of the embedded system was to successfully estimate the motion variables relevant to the occurrence of falls, tips and similar risky situations. Currently, 
off-line smoothing techniques based on Kalman filter concepts allow for optimal estimation of angles in the longitudinal direction, roll, and in the lateral direction, pitch.

Results from both predefined experiments with known outcomes and data collected from actual wheelchair users during pilot and final deployment stages are presented and discussed. 


\section{Chapter 1}

\section{Introduction}

\subsection{Motivation}

The motion logger was developed with the purpose of studying the risk factors of wheelchair-related falls among the elderly. The research included the costs and

consequences of those events, [1]. Combined with follow-up phone interviews, data collected by the data-loggers is used to identify intrinsic factors, extrinsic factors and their relationship with demographic information.

The research effort was expected to identify previously unaccounted factors, which predispose wheelchair users to fall or become injured. Fall prediction models can be created and used to develop an evidence-based, subject-centered wheelchair-falls prevention program. The main objectives of characterizing Wheelchair-Related Falls in Elderly Veterans study are detailed below, [1]:

- Describe the incidence and prevalence of wheelchair tips, falls and fall related injuries.

- Describe the epidemiology of the wheelchair tip or fall event.

- Create models for predicting wheelchair tips, falls and fall-related injuries for elderly persons who use a wheelchair for mobility. 
- Determine healthcare utilization and direct costs associated with wheelchair related fall injuries.

- Describe subject perceived short and long term consequences of wheelchair related falls.

The main advantage, which the motion sensing system provides, is the absence of a requirement for user interaction during data collection related to the detection of tip and fall events. After the data is acquired, and analyzed off-line, the developed software application generates a report, which includes the time and type of event detected.

\subsection{Background}

A multitude of risk factors associated with falling has been reported for the elderly. Many of these risk factors are not directly applicable to persons with a Spinal Cord Injury, (SCI), who use a wheelchair for mobility. However, the Epidemiology and Cost of Falls for Veterans with a Spinal Cord Injury was carried out between April 2004 and March 2008, [2].

The objectives of the study were:

- Describe the incidence and prevalence of tips, falls and fall-related injuries for SCI subjects.

- Describe the time, place, activity and epidemiology of the person experiencing the fall event. 
- Describe any injuries associated with a fall including the mechanism, nature of the injury, severity of injury and treatment required.

- Determine the risk factors related to falls and fall-related injuries in SCI and develop a model for predicting falls and fall-related injuries in SCI.

- Determine healthcare utilization as well as direct and indirect costs associated with fall-related injuries in SCI.

- Describe subject-perceived short and long-term consequences of falls in SCI.

Data was collected through subject surveys, medical records and Veteran Administration, (VA), databases. Risk factors characterization included user characteristics, wheelchair features, wheelchair activities and physical environmental data. Tips, falls and injuries were tracked via monthly follow-up calls.

Recruitment ended on April 1, 2006 with a total of 702 subjects. Data for analysis was provided by 659 subjects of the recruitment group. Data collection was completed on March 31, 2007. A total of 553 fall events were reported by 204 individuals. Ninety five, (95), of the study subjects were injured as a result of a fall. Regression models showed that fall occurrence was predictable based on factors, which occurred prior to the fall event. These factors include increased pain in the preceding two months, increased alcohol use, experiencing a previous fall, shorter wheelchair length and fewer years of SCI. 
Injurious falls were predicted by increased pain in the previous two months, experiencing a previous fall, existence of a non-flat home entrance and a diagnosis of arthritis.

While much is known about falls in the elderly, there is a lack of understanding of the epidemiology of wheelchair-related falls. The research presented in this thesis was expected to identify previously unaccounted factors, which predispose persons with SCI to falls and fall-related injuries. This research resulted in the creation of a model for predicting falls and fall-related injuries in SCI. The predictive model can be used to develop intervention strategies, which target modifiable risk factors. Eventually, the predictive model developed during this research can be tested with other vulnerable veteran populations. Findings from this research can be used to develop an instrument to identify fall risk in persons with SCI.

\subsection{Objectives and Scope of the Research}

The development of the motion-logger was motivated by the need for a device to support the research associated with tips and falls of wheelchair users. A logging and estimation system must provide accurate information related to the motion and attitude of the wheelchair in order to successfully determine risky situations. The final solution needed to accurately estimate relevant motion information so that appropriate conclusions could be drawn from the research. 
The developed motion-logger aids in the collection of information relevant to the research such as the time when every tip and fall was detected and an angle estimation process. This information, combined with demographic records, constitutes a comprehensive source of information for the research. Precision of the angles estimation process can be estimated through predefined experiments whose outcomes are measurable. The tip and fall detection algorithm must be capable of identifying causes and specific patterns. In addition, and very importantly, the algorithm must reduce the number of false positives.

System reliability was initially determined by comparing the results, obtained after executing the algorithm, to collected data and from the feedback provided by the wheelchair users who participated in the short-term pilot study. 


\section{Chapter 2}

\section{Related Research}

\subsection{Research Projects}

Wheelchair usage statistics are very important for investigating users' behavior and for evaluating safety and reliability. Activity levels of wheelchair users are critical factors in early problem detection and timely intervention, [3]. Reduced mobility is usually related to social isolation, unemployment, obesity, cardiovascular disease and pressure ulcers, [3].

Many research projects are being developed, which deal with attitude and position estimation, based on IMU measurements. In the following sections, several projects dealing with motion estimation and most of which are wheelchair-related, are presented. At the end of the chapter, the solutions are analyzed in terms of their advantages, disadvantages and suitability to the objectives presented previously.

\subsubsection{Wheelchair Usage Monitoring/Logging System}

A system was attached to a wheelchair, Ding et.al, for determining several usage statistics such as distance traveled, localization information, seat positions and time spent on the wheelchair, [3]. Using wheel rotation measurements, the velocity, distance and 
number of stops were estimated. A GPS board was used to collect data about traveling information. A potentiometer was used to measure seat tilt values and to obtain the time users stayed in various seating positions. The system consisted of a Wheel rotation logging module, a GPS logging module and a seating posture logging module.

The wheel rotation logging module records time-stamps of wheel rotations and reports activity related data such as traveling distance, direction, velocity and the number of stops. It uses a pendulum with a small magnet at the bottom, which sweeps over three reed switches, which are separated by 120 degrees. Time-stamps of each sensor event are stored in a flash memory. The wheel rotation logging module is pictured in Figure 1.

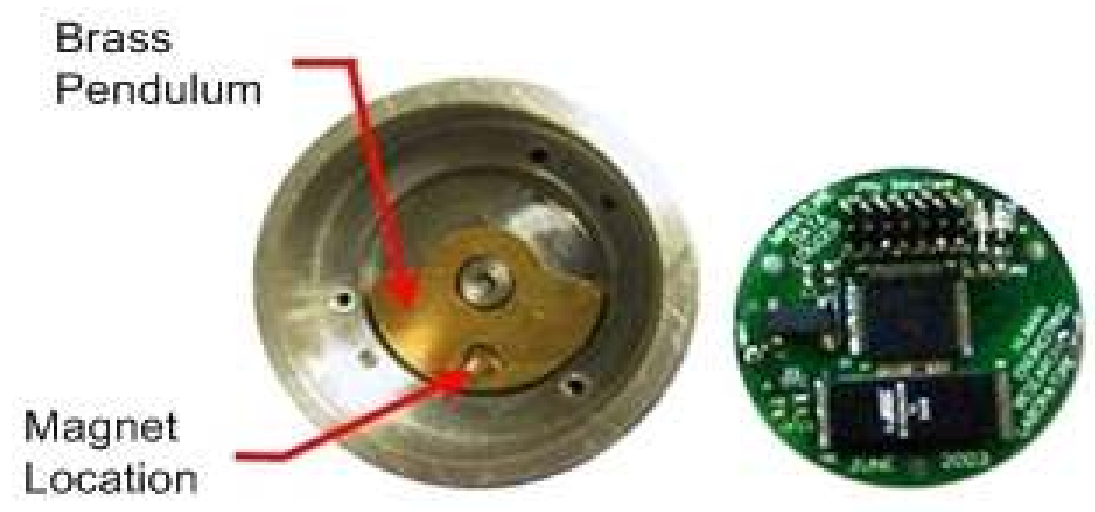

Figure 1: Wheel Rotation Logging Module, [3]

A GPS logging module was used to collect traveling information about wheelchair users. The physical location provides information on users activities and community participation. Since the GPS signal can be lost in indoor environments, it was combined with wheel rotation information for purposes of comparison. A data 
comparison is necessary in order to determine the difference between the activity levels of wheelchair users in indoor and outdoor locations in terms of total time, distance and average velocity.

The seating posture logging module includes seven sensors. Three tilt sensors measure the angle of the base, the angle of the seat and the angle of the back. Three pressure sensors, under the seat cushion, are used to determine the chair occupancy and weight shifting activities. A potentiometer measures the seat elevation. The seating posture logging module is pictured in Figure 2.

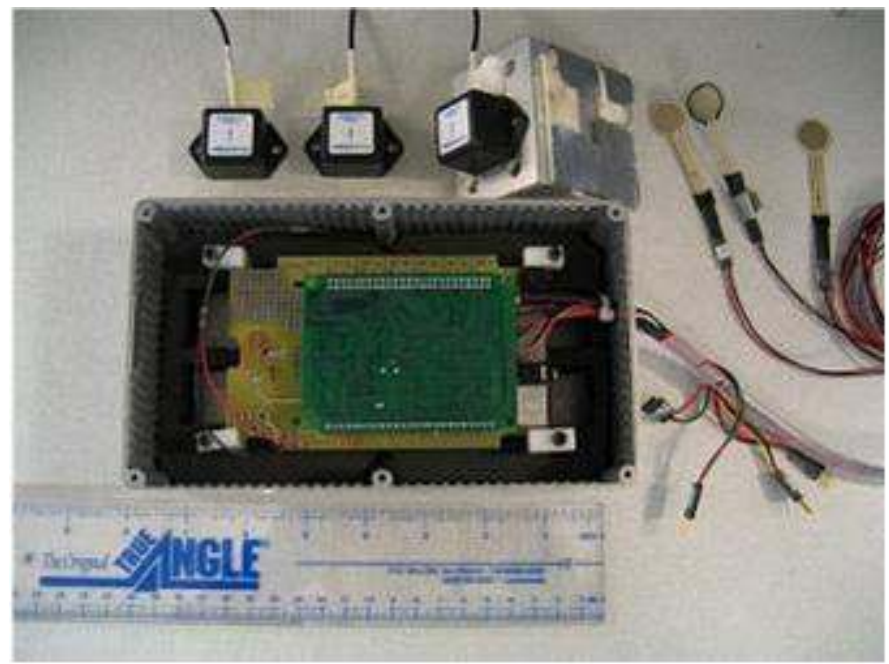

Figure 2: Seating Posture Logging Module, [3]

The output of the system includes the frequency and duration of accessing each seating feature, common tilt angles and the percentage of time spent in different seating positions.

Even though this system provides information regarding distance, speed and time, it is not accurate in indoor locations due to the fact that it relies on GPS data for 
estimating the variables. This fact alone would make the system unsuitable for the situation addressed in this research since the study with wheelchair users requires continuous logging under the subject's regular conditions. Additionally, although the pressure sensor determines chair occupancy and would reduce the probability of a false positive, when the wheelchair is not being used by the subject. However, it does create the need for connections between the sensor and the main board. This connection would increase installation complexity and wheelchair intrusion. Both situations are recommended to be and, indeed, should be kept at a minimum. The same issue is present with the wheel rotation logging module as additional connections and specific installation requirements are identified.

\subsubsection{Monitoring Seat Feature Usage among Wheelchair Users}

In order to measure the usage of seat features such as seat tilt and backrest recline in powered wheelchairs, a Seat Feature Data Logger, (SFDL), was attached to 12 subjects' wheelchairs for 10-14 days. These seat features help managing pressure ulcers and posture adjustment. Among people with complete quadriplegia and paraplegia, it is estimated that $60 \%$ and $50 \%$ respectively develop pressure ulcers, [4]. Many users are not able to independently relieve pressure on the buttocks and thighs, In some cases, the subject might not have sensation. Studies have shown that the use of seat tilt can significantly reduce static seating pressure, [4]. 
The SFDL system was designed to measure how often wheelchair users use the seat tilt, backrest recline and seat elevation during daily activity. The use of seat features was quantified by calculating the frequency and duration of accessing each feature.

The SFDL system design included a programmable data logger, three tilt sensors, three pressure sensors and a linear position transducer. The tilt sensors monitored seat tilt and backrest reclines, the pressure sensors monitored seat occupancy and the linear position transducer monitored seat elevation. Data analysis was carried out in MATLAB. The average frequency of accessing specific tilt angles per day are presented graphically in Figure 3.

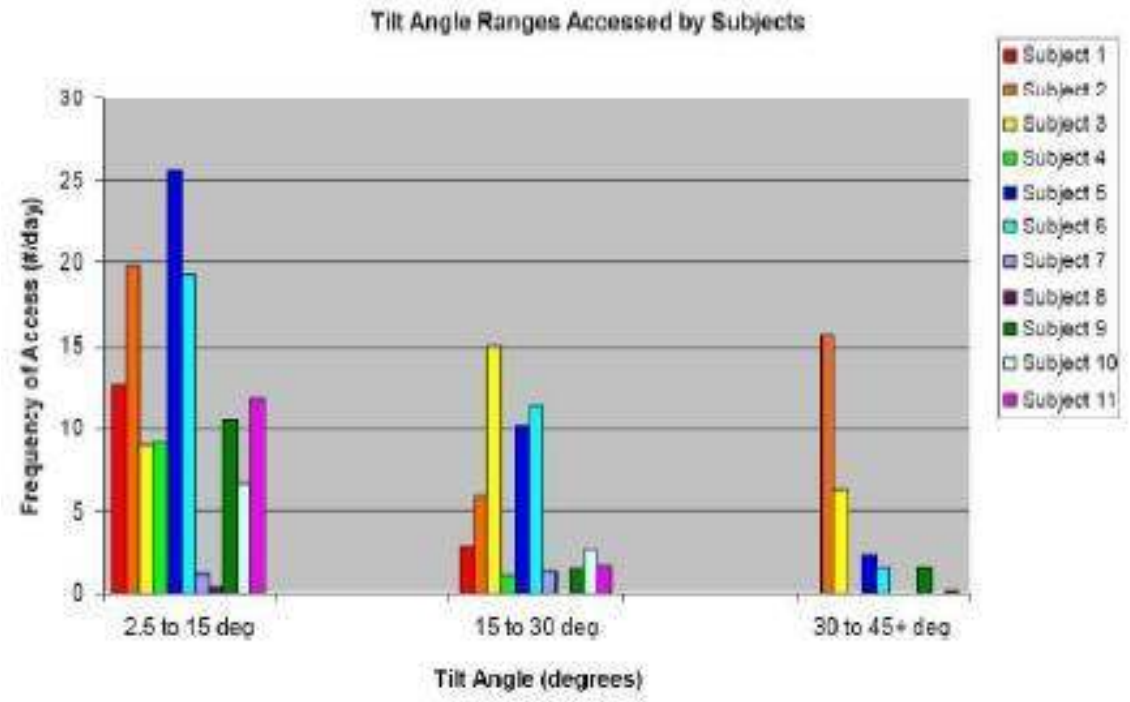

Figure 3: Seat Tilt Angle Ranges Accessed by Subjects, [4] 
The average frequency of accessing specific recline angles per day is presented graphically in Figure 4.

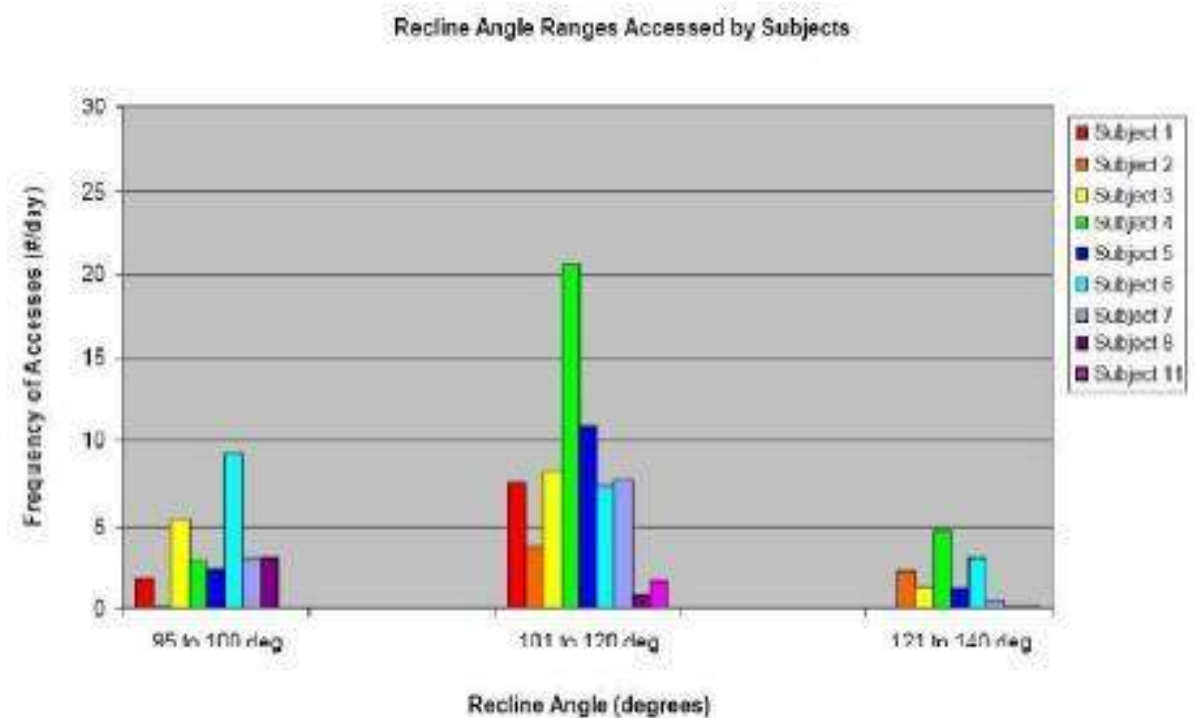

Figure 4: Backrest Recline Angle Ranges Accessed by Subjects, [4]

Results indicate that wheelchair bound subjects used small tilt angles to improve posture and comfort instead of relieving pressure by shifting weight, according to previous wheelchair user studies, [4]. Reclined positions may be undesirable since they are associated with shearing on the back, which, at the same time, is a risk factor for pressure ulcer development. Additionally, reclined positions are not recommended when stability is a concern. It was found that users accessed recline 6 times per day fewer than they accessed tilt. Subjects accessed tilt and recline together slightly more than half the time the subjects occupied their wheelchairs. Combining tilt and recline leads to maximum reductions in peak pressure, which is superior to the reductions realized when each feature is used alone, [4]. Future work should include the development of a SFDL 
system, which is combined with coaching software to incorporate specific usage profiles recommended by clinicians.

The system developed during this research was very similar to the one described in Section 2.1.1. Therefore, it offers the same difficulties. There is a difference between the two research efforts. The research referenced in Section 2.1.1 deals with measuring motion statistics such as distance traveled, average speed and usage time. The focus for this research was directed toward the determination of how often wheelchair users access features such as backrest and seat recline. These objectives were established since these features have been found to have a significant effect on pressure management and posture adjustment.

\subsubsection{An Indoor Localization Aid for the Visually Impaired}

Hesch and Roumeliotis proposed an indoor localization system for the visually impaired, [5]. The system used a laser range finder, a three-axis gyroscope and a pedometer mounted on a cane to provide position and attitude information.

The laser and gyroscopes provided attitude information. The pedometer measured the user's walking speed. The estimation scheme used a two-layered extended Kalman filter. The first layer, based on relative attitude measurements inferred from the laser and 3-axis gyroscope rotational velocity measurements, produced and estimate of the 3-D attitude of the cane. The second layer, integrated corner features, which were extracted from the laser data and the pedometer linear velocity measurements, to estimate the 2-D 
position of the user. Considering that many of the primary structural planes such as the floor, the ceiling and the walls of a building are perpendicular. If information about the location of environmental features such as corners is known, the implemented method provided a reliable localization estimate of a person traveling indoors. The necessary hardware to be mounted on the cane is pictured in Figure 5. The sensors were interfaced with a laptop via USB, RS-232 and Bluetooth.

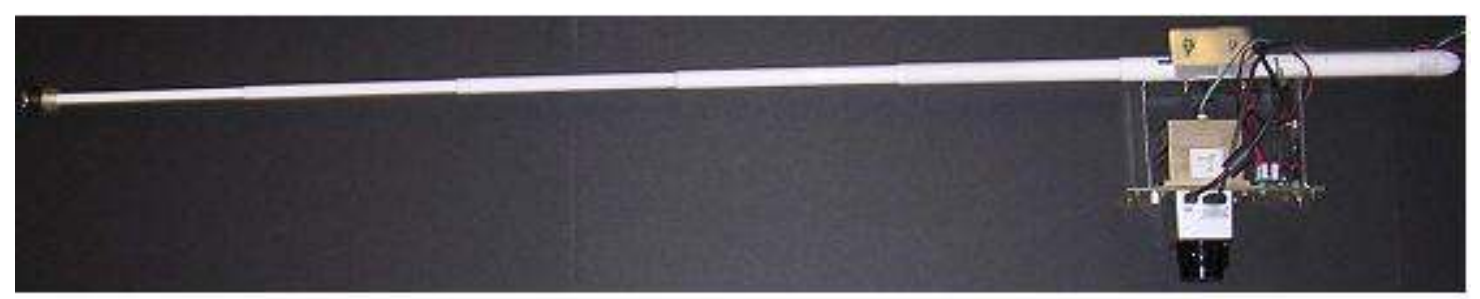

Figure 5: White Cane and Hardware, [5]

Future work planned includes generating routes between indoor locations and the development of a feedback system to provide the person simple directions. Furthermore, the researchers plan to use the white cane system in cases where maps for the building are not available.

The white cane system calculated 3-D attitude information in a similar way as the solution obtained during this research. However, the white cane approach presents several difficulties. Location information is obtained from the laser based on known building maps. Since wheelchair users' location will certainly vary during daily activities, continuously accurate location, determined with this system, will not be possible. In addition, the sensors were interfaced with a computer using USB, RS-232 and Bluetooth. 
One of the crucial requirements for the design was that all users do not necessarily have computers available to them. Therefore, the data must be stored for relatively long periods of times, possibly for several weeks, before it can be downloaded to a PC. This requirement made the white cane system inappropriate as a possible solution for this research.

\subsection{Commercially Available Products}

In this section, a summary of the research literature covering some of the already commercially available products is presented as per achieving the objectives mentioned in chapter 1 .

\subsubsection{HOBO Pendant G Acceleration Data Logger}

The HOBO Pendant G Acceleration Data Logger is a commercially available motion-data-logging device, which stores measurements from a three-axis accelerometer in a $64 \mathrm{~KB}$ EEPROM memory, [6]. The information is downloaded to a computer through a USB interface. With the help of software, included with the equipment, tilt information is computed and displayed graphically. The data logging device is pictured in Figure 6. 


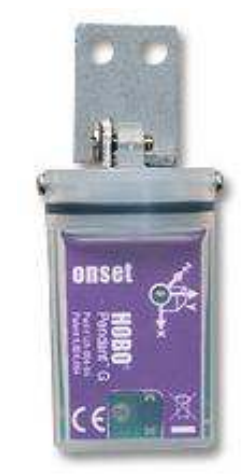

Figure 6: HOBO Pendant Acceleration

Data Logger, [6]

The data logger can be used for a wide variety of experiments, demonstrations and field studies by measuring dynamic acceleration due to motion, vibration or shock. Since the data logger also detects static acceleration due to gravity it is possible to measure tilt, activity and inclination.

Potential applications for the data logger device include monitoring sports activities, medical therapy compliance or any other activities or processes where 3dimensional motion is present and the data is required to be stored for analysis.

The HOBO Pendant Acceleration Data Logger could have been obtained at a very favorable price, $\$ 69$, which would have reduced engineering development cost. However, it could not fulfill most of the requirements imposed for the motion logging device specified for the wheelchair necessary for this research. Due to its limited storage and battery capacity, the HOBO Pendant is only applicable to short time duration experiments. The HOBO data logger can only log data up to 40 minutes when sampling at $10 \mathrm{~Hz}$, which was the proposed system sampling frequency. Furthermore, gyroscopes are necessary for accurate estimation of dynamic roll and pitch. 


\subsubsection{The "Black Box" - A Powered Wheelchair Usage Monitor}

The wheelchair "Black Box", data logging system, was developed to monitor powered wheelchair usage over a 40 day cycle, [7]. The device monitors current drain through the serial link between the $12 \mathrm{~V}$ batteries of a powered wheelchair. The battery charge or discharge is averaged over 30 minute intervals and the information is stored in non-volatile memory on board the data logger. The data logger is controlled and read by a computer through a serial link via a dedicated interface. The data is displayed graphically in a DOS format as one week per screen, or in a tabular form as one day per screen.

The data logger was very successful in reducing the high rate of battery failure by identifying inappropriate charging procedures. In addition, when fitted to trial wheelchairs, the data logger provided valuable information on wheelchair usage patterns for the prescribing therapist.

This data logging device is useful for battery monitoring and can provide some rough motion time estimation. However, the device is based on current consumption information, which allows only rough estimates of acceleration and speed. The Black Box data logging device system does not directly provide any specific information on motion variables. Therefore, tips and falls events detection, as required in the wheelchair-related falls study, would not be achievable with the Black Box device. 
Table 1 includes a comparison between the available data-loggers presented in this section. Table 1 presents reasons for the unsuitability of each device to function as part of the required solution for this research.

Table 1: Comparison between Referenced Projects

\begin{tabular}{|c|c|c|}
\hline Design & Advantages & Disadvantages \\
\hline $\begin{array}{l}\text { Wheelchair Usage } \\
\text { Monitoring/Logging } \\
\text { System }\end{array}$ & $\begin{array}{c}\text { Directly measures angle of } \\
\text { seat, base and back }\end{array}$ & Installation complexity \\
\hline Monitoring Seat Feature & Determines backrest and & Reliability on GPS \\
\hline Usage among & seat recline features & information for position \\
\hline Wheelchair Users & accessing frequency & tracking \\
\hline An Indoor Localization & & Requires computer to \\
\hline $\begin{array}{l}\text { Aid for the Visually } \\
\text { Impaired }\end{array}$ & 3-D attitude estimation & $\begin{array}{l}\text { download information } \\
\text { continuously }\end{array}$ \\
\hline $\begin{array}{l}\text { HOBO Pendant G } \\
\text { Acceleration Data } \\
\text { Logger }\end{array}$ & Tilt estimation & $\begin{array}{l}\text { Short-term logging at } \\
\text { high sampling frequencies }\end{array}$ \\
\hline The "Black Box" - A & Reduce battery failure by & System does not provide \\
\hline Powered Wheelchair & identifying inappropriate & specific motion \\
\hline Usage Monitor & charging procedures & information \\
\hline
\end{tabular}




\section{Chapter 3}

\section{Systems Engineering}

\subsection{Customer Needs, Requirements and Challenges}

The development of the "Motion Logger" was motivated by the need for a device to support research into tips and falls by wheelchair users. In order to support the research of Wheelchair-Related Falls, a logging system was required to detect and report tips and falls by wheelchair users. Several considerations were detailed by the customer for the proposed solution. First, the solution must provide accurate information regarding tips and falls events. Second, the system must work on different types of wheelchairs possessed by the users. Third, minimum wheelchair user intervention was highly desirable in order to reduce user research participation rejection. Finally, cost was a key factor since final deployment could require the assembling and installation of up to 200 devices.

A logging and estimation system must provide accurate information about the motion and attitude of the wheelchair in order to successfully determine the existence of risky situations. The final solution needed to accurately estimate relevant motion information so that appropriate conclusions could be drawn from the research. 
The system needed to be tested and implemented under real conditions.

Therefore, the data logger had to possess requirements such as:

- No relevant events should be missed,

- Adaptability to a variety of wheelchair types and users,

- Robustness,

- Simple use with minimal user interaction,

- Low price.

With regard to the results obtained from analysis of raw data, it was essential to accurately estimate relevant motion variables. Accurate motion data was mandatory for the application of pattern recognition techniques so that events were not missed while avoiding false positives. In addition, the conditions, which should be taken into account, were:

- Users might not have computers accessible to them to be able to download/transmit data.

- Data must be stored in the device before it could be obtained for analysis by the research team. Some type of storage device replacement must be implemented so that the logging process was not interrupted.

- Deployment of logging devices could occur in large quantities over a wide variety of wheelchair types.

Figure 7 includes pictures of the different type of wheelchairs owned by the users who participated in the study. 

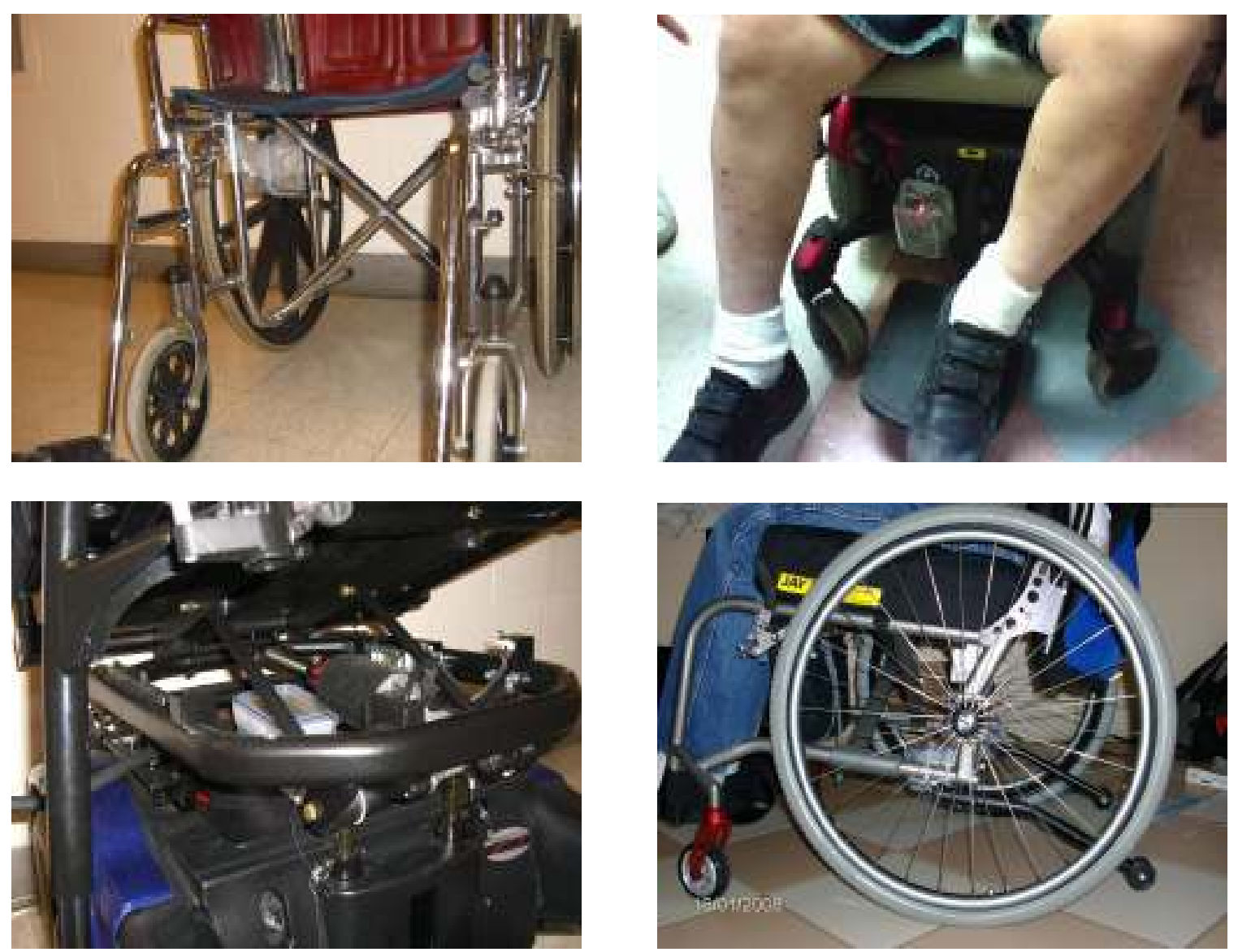

Figure 7: Different Wheelchair Types for Device Installation

\subsection{Functional Analysis}

In order to fulfill all stated requirements the main components of the solution were identified as:

- Store measurements collected from sensors,

- Estimation of motion variables,

- Algorithm for the determination of fall/tip events.

The computational power required for data analysis and the energy consumption of the data logger were the most important reasons for implementing the estimation and 
fall/tip detection algorithms in a PC. Executing the algorithms in an embedded device would have reduced the amount of memory required since only the relevant events and time-stamps would have been stored in the memory. However, such a decision would have increased the total power consumption. Power consumption would increase due to the need to process the detection algorithms within the sample time, which would require the microcontroller to run at higher frequencies. In addition, the raw data would not have been available to the research team for further analysis. The availability of raw data is useful for comparing results after executing different detection algorithms. In addition, raw data availability is also necessary to carry on other research studies in the field.

This system architecture leverages on the computational power of the PC and its large storage capacity. It also provides for minimizing the complexity of the data-logging device, which reduced its cost.

Following a systems engineering approach for the design of the data logging device yielded the system block diagram presented in Figure 8. 

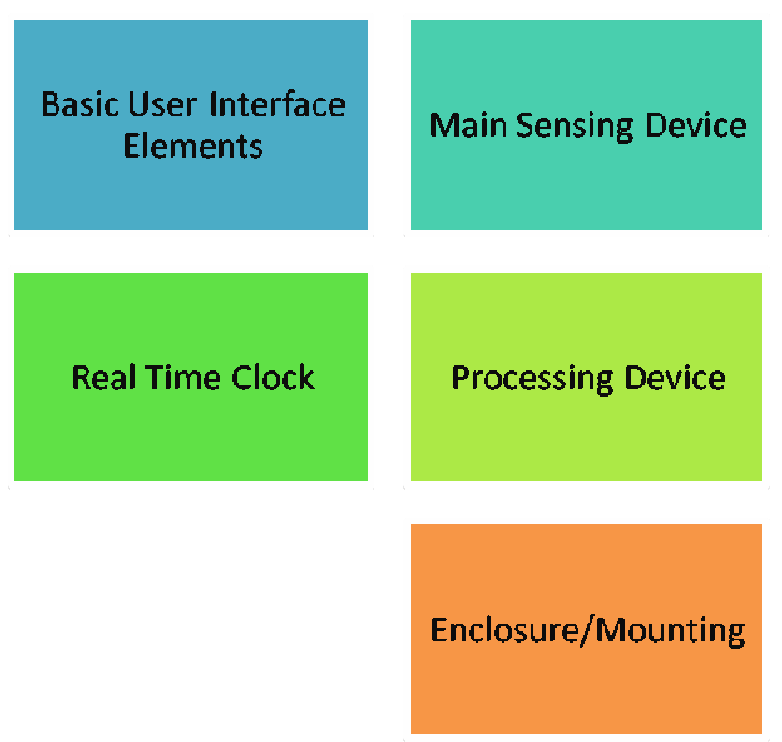

Figure 8: Proposed Solution Block Diagram
Memory

Power Subsystem

\subsection{Alternative Implementation Solutions}

Previous studies aimed at the evaluation of risk factors of tips and falls related events, by wheelchair users, have relied on data collected through direct interview of the subjects, [2]. These studies also provided data related to the person, time, place, epidemiology of each event and activity. Information regarding subject-perceived effects was also collected during the studies. However, the main difficulty encountered with relying solely on the subjects, as the source of information, is the fact that they might forget and/or underestimate important events when reporting in person or by phone to the researcher.

In order to improve the subjects tips and falls events logging process, one of the possible solutions considered was to install a small switch or button. The switch or button should be pressed any time a relevant situation occurred. The switch would be 
connected to a logging device whose functions would include recording the data in some type of memory. The data would include time-stamps for future data analysis. The main advantages, which this system offers over previously implemented "Motion-Loggers", are its cost and simplicity. However, the switch-based option was not used due to its dependence on user interaction as the only source of information. Installation of an external switch, which would be easily accessible to the user, and a logging device on the wheelchair would have required additional hardware. Therefore, the system would be more susceptible to externally induced connection problems and would increase installation times and complexity.

The most important consideration regarding the memory type for the data logger related to how the memory card data was going to be unloaded for analysis. Users hold the device for several months. This requires data to be stored for long periods of time in the device before it can be delivered to the research team for analysis. Therefore, while performing analysis, a replacement storage device must be delivered to the user so that data logging is not interrupted. SD memory cards and EEPROM memories were two types of memories considered as possible solutions: The main advantage, which the SD memory card solution offered, was the ease of management of the exchange process for the user. On the other hand, an EEPROM memory requires users to interact with integrated circuits whose pins can easily be damaged during attachment and detachment to the main board. Finally, users are more familiar with handling SD memory cards, such as the ones used in digital cameras, than they are with using integrated circuit devices. 
Whether or not to execute the estimation and tips and falls detection algorithm in the device was an important consideration. Higher processing loads in the data logger require the microprocessor, embedded in the device, to run at faster speeds, which increases power consumption. However, performing the processing algorithm in the PC takes advantage of its computational power and its different results presentation options. Furthermore, having the raw data allows the research team to evaluate different alternatives, over the same collected data, for comparison purposes.

The "Motion-Logger" system was selected as the final solution due its high reliability on data collection. In addition, the motion logger offers the possibility of implementing a continuous data logging process. Furthermore, no user interaction is required for relevant data reporting.

\subsection{Analysis of Alternatives and Comparison}

In accordance with the block diagram presented in Figure 7 and the mandatory requirements, it was decided that the data logger should execute the sensing function and store measurements from the sensors. The main processing algorithms were executed in the PC.

In order to optimize spare memory usage, an appropriate sampling rate had to be chosen for every sensor and its value stored in memory. The sampling rate was set to 10 samples/second. The sampling rate will be explained in more detail in Section 4.3. Additionally, a rechargeable battery system was selected since the study required data 
logging for a long period of time per user. A non-rechargeable system was impractical because of its cost and battery replacements requirements, which would have involved more user interaction with the device.

\subsection{Final Design Decisions}

After a careful evaluation and comparison of several alternatives, Sparkfun's

Logomatic V1.0 was chosen for hardware implementation of the initial proposed system. The Logomatic contains the Philips LPC2138 microcontroller and a Secure Digital Card, (SD), socket, [8]. The SD memory was chosen due to its memory capacity and lowpower specifications. In addition, it also provided for easy installation and card removal. The motion sensing function in the data logging device was realized by an Inertial Measurement Unit, (IMU), containing 3-axis accelerometers and 2 gyroscopes, which were obtained from the same vendor, [8], [9]. In addition, temperature and battery voltage were measured for device characterization and for monitoring power supply behavior with the help of a custom designed circuit. A diagram of the device and its main components are presented in Figure 9. 


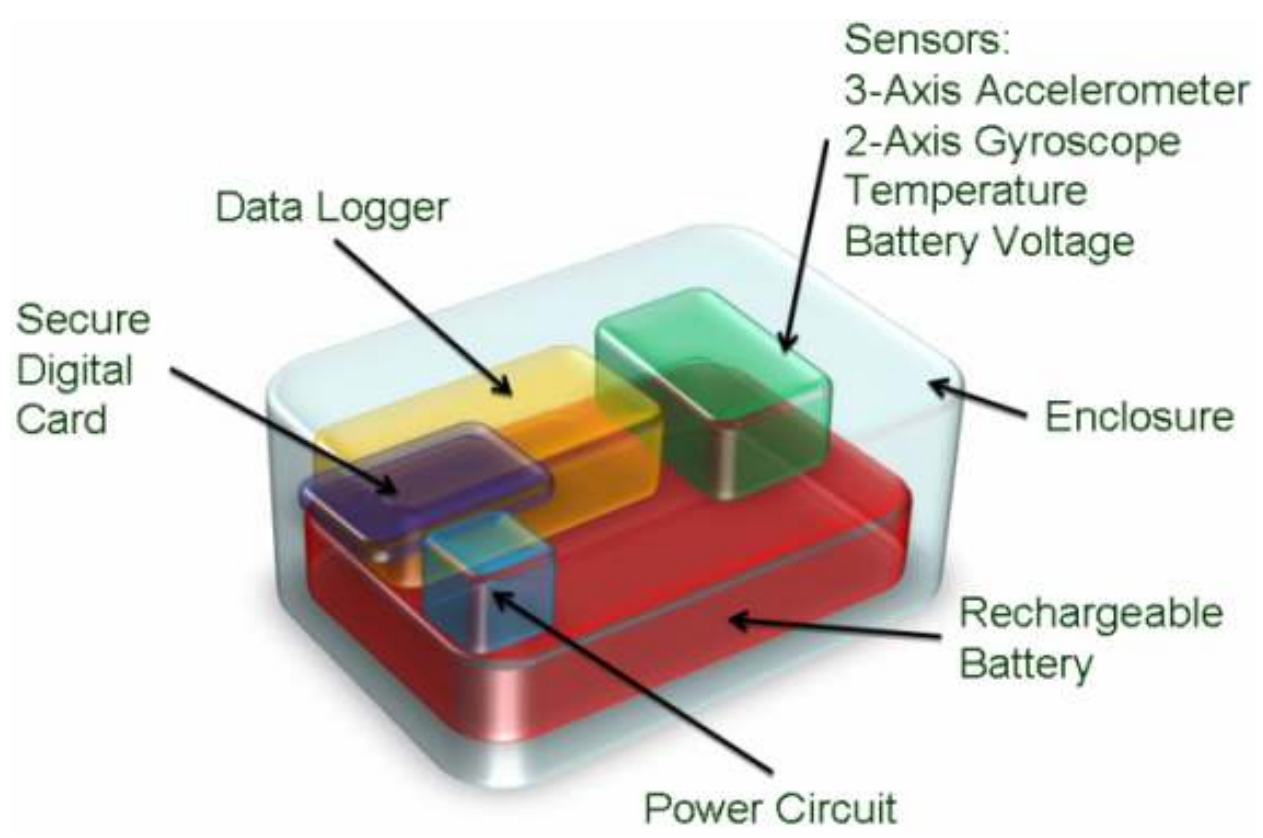

Figure 9: Embedded platform

The device was assembled in a water-tight plastic box for ease of accessibility.

Battery charging and memory replacement were accomplished by removing the top of the plastic box.

The device was rigidly attached to the wheelchair in order to capture the motion variables with high fidelity. The data logger could be attached to any flat surface of the wheelchair using Velcro. The Velcro was tested in the laboratory and demonstrated sufficient strength at a very low cost. Pictures of the embedded system, with and without the removable cover, are presented in Figure 10. 

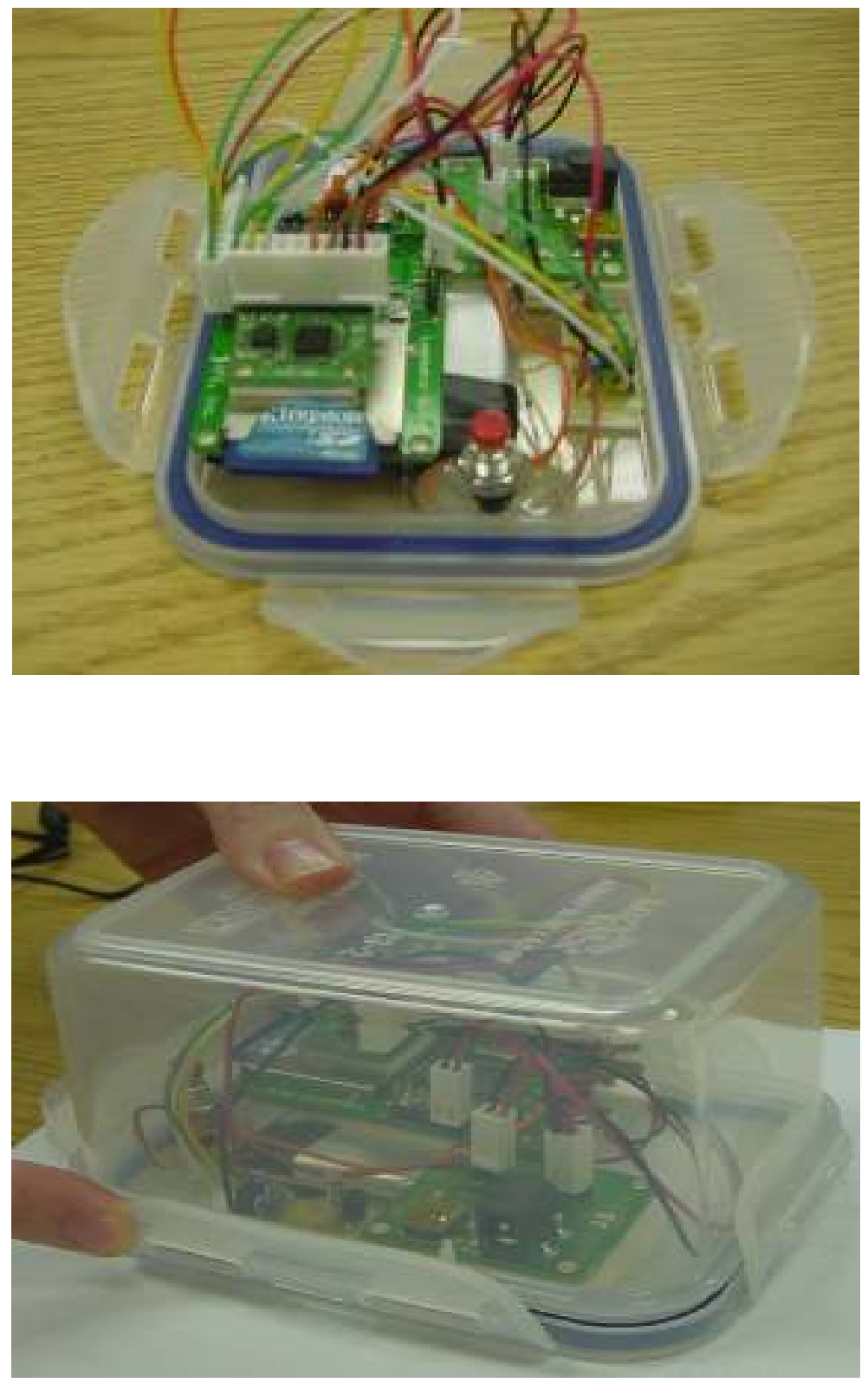

Figure 10: Embedded Device

Figure 11 illustrates how the data logger was attached to the wheelchair using Velcro, which contains a sticky-back tape for adhering to each surface. 


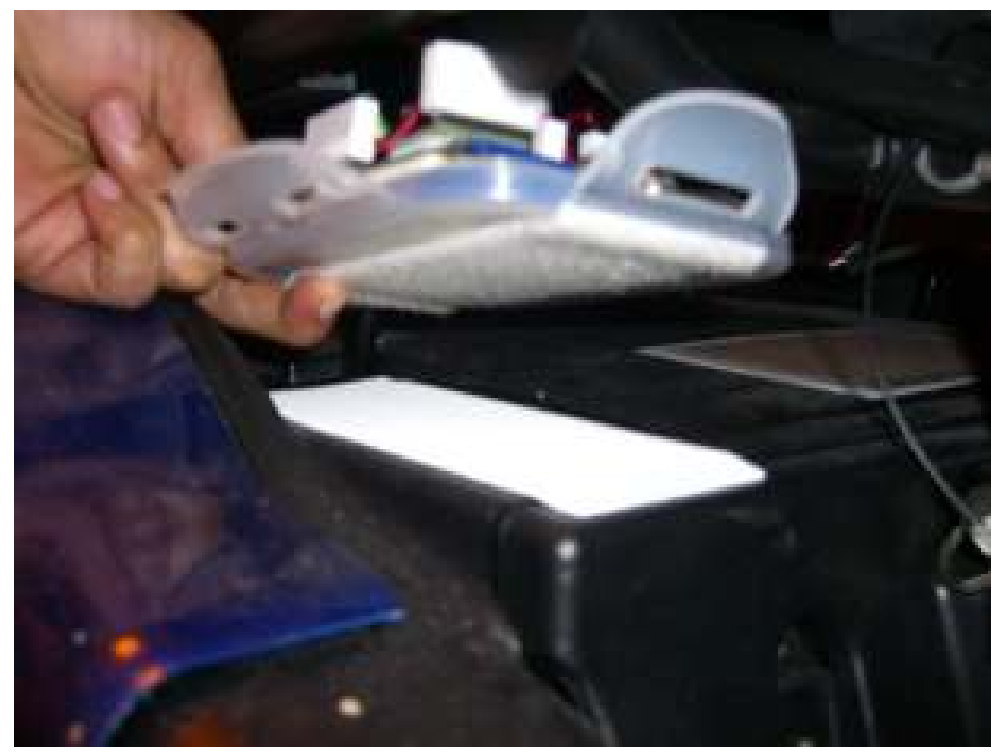

Figure 11: Velcro Adhered to the Wheelchair and the Data Logger for Coupling

Figure 12 displays two pictures of the device attached to one of the powered wheelchairs. 

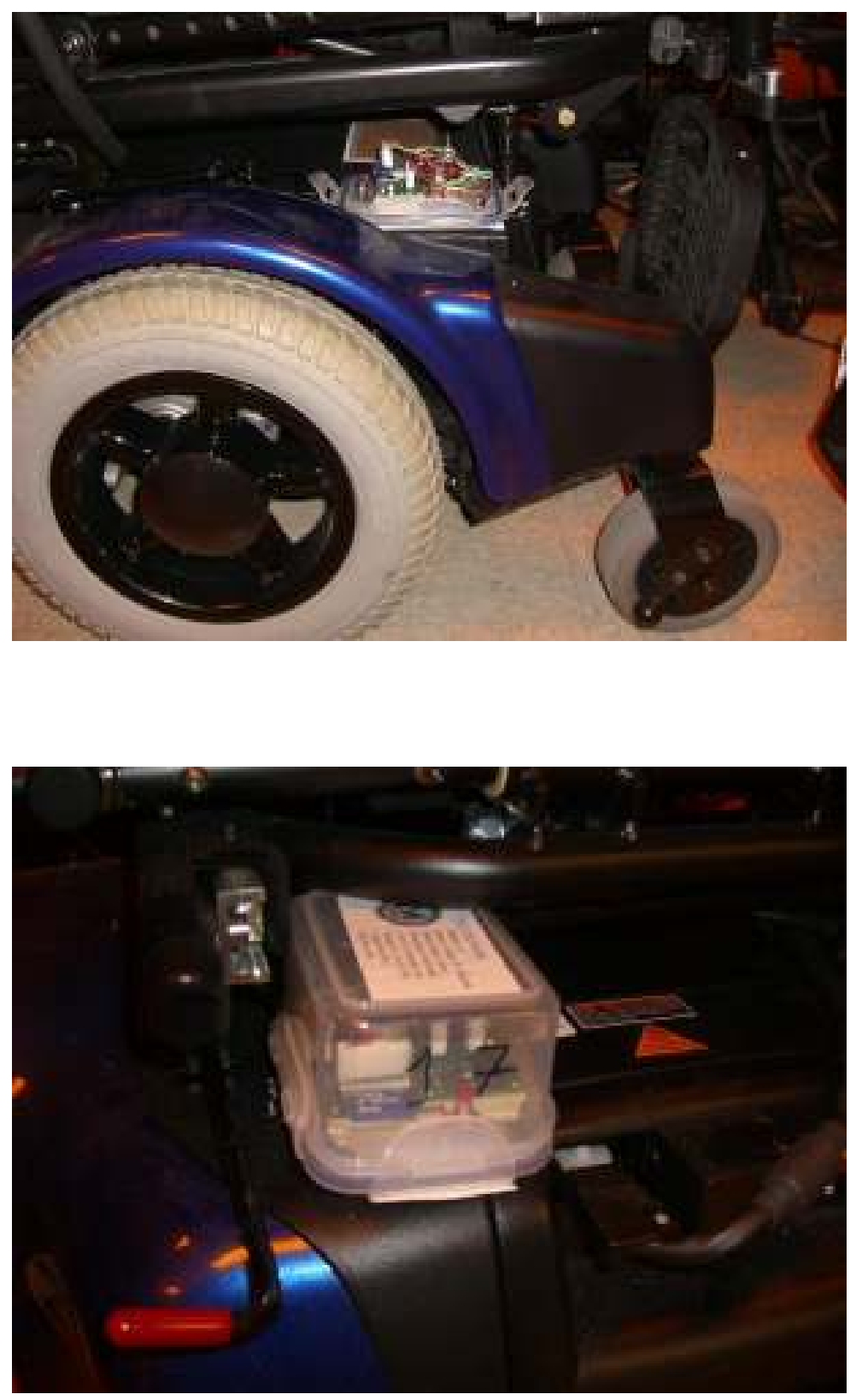

Figure 12: Data Logger Attached to a Wheelchair 


\section{Chapter 4}

\section{Embedded Application Development}

\subsection{Description}

Acceleration and angular speed information was processed by an embedded application so that measurements were stored only when the wheelchair moved. This particular functionality was important for saving storage space and energy. Code for the custom embedded application was written using available functions for the Logomatic's firmware. The function source code was provided by the vendor. Features incorporated in the customized embedded application were:

- Real time multichannel signal processing for selective data storage,

- Time-stamping,

- Reading input elements for user interaction,

- Battery and temperature monitoring.

Figure 13 presents the flow chart of the embedded application implemented in the microcontroller after modifying the Logomatic firmware. The code starts by initializing the microcontroller through the declaration of functions and global variables. 


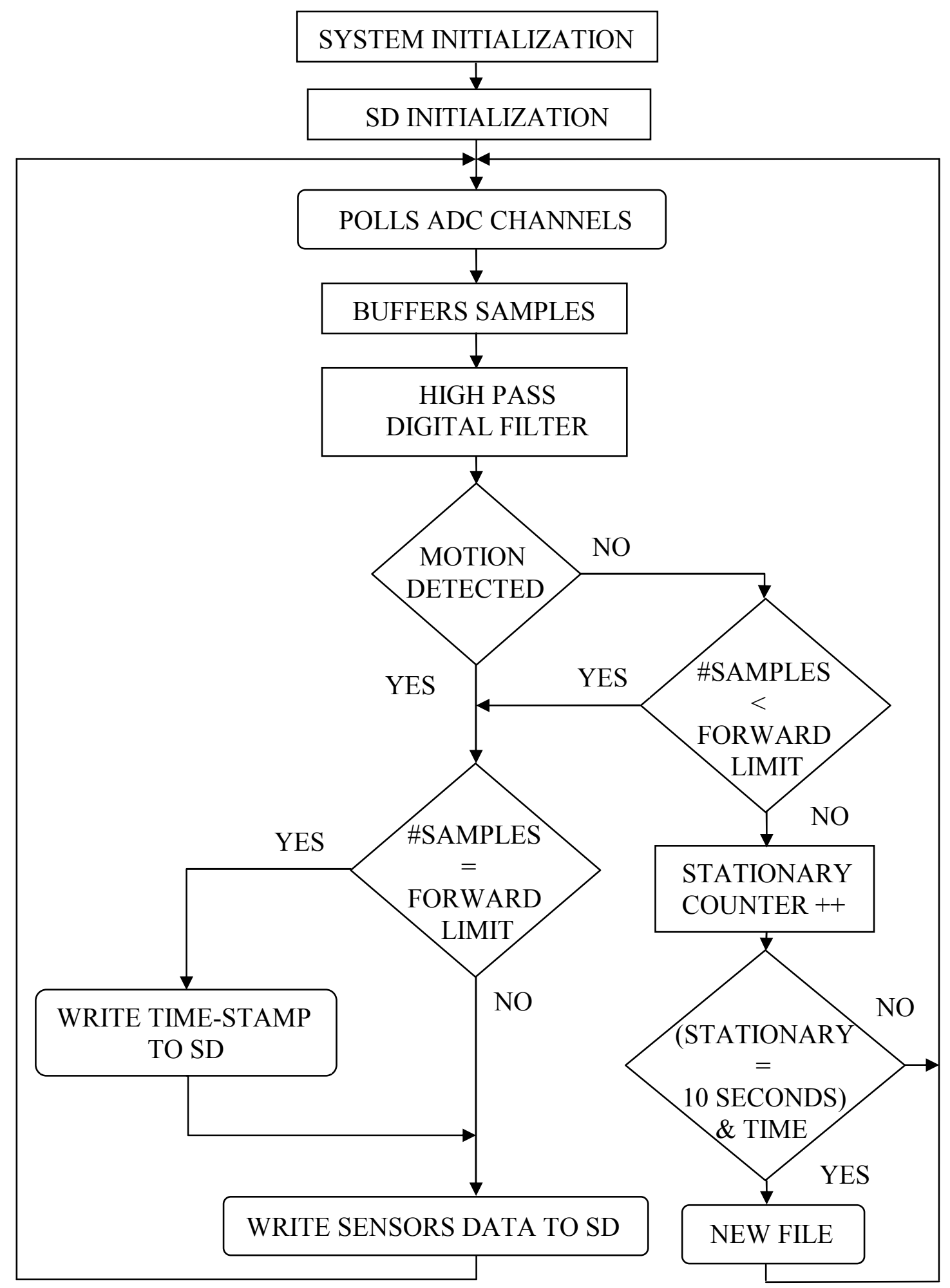

Figure 13: Embedded Application Flow Chart 
The clock is set using the Phase-Locked Loop, (PLL). I/O pins are defined during the Real Time Clock, (RTC), configuration and initialization and setting the Analog to Digital Converter, $(\mathrm{ADC})$, channels are some of the main functions executed during the microcontroller configuration process. After the SD memory card file system is set to FAT16, the main process starts by continually polling the ADC channels, which are connected to the sensors. The ADC channels collect data from the temperature, voltage, accelerometer and gyroscope sensors.

In order to establish a reference for the stationary state prior to the detection of motion, five samples from each sensor are stored in a buffer for the high pass digital filter. This data is written to the SD card once the data logger detects any motion. Therefore, whenever a new frame is generated in the memory card the first five samples, which is equivalent to half of a second of sampling, are stored. The reference samples are followed by the time-stamped samples, which are generated due to sensed motion.

A high pass, (HP), digital filter was implemented in real time. If the HP filters output was higher than a threshold the system triggered the writing process for the SD card. The motion threshold was established very low to insure even low vibration would be detected. Therefore, the only time data, coming from the sensors, was not logged in the memory card was when the system was completely stationary. Additionally, after a stationary state is detected several samples, set by the forward limit variable, were logged in order to avoid losing any type of relevant information due to short stationary states. Real Time Clock information, time-stamp, as well as temperature and voltage values for 
battery monitoring, were logged to memory whenever activity was detected after being in a stationary process. Such data was logged at the beginning of a new frame. The data logging procedures are depicted in Figure 13.

In order to avoid creating large files in the memory card, which would slow the data processing of the $\mathrm{PC}$, two new files were created daily to keep file size more manageable. Another important consideration supporting the idea of creating several short files, rather than a large one, is the fact that if a file is corrupted and unreadable not all the data would be lost.

\subsection{Signal Analysis}

The signal processing algorithm is based on a high-pass digital filter applied to each sensor output. The output of the digital filter determines whether a change has occurred, which should trigger the memory writing process. The high-pass digital filter was designed and validated using MATLAB. Relevant frequency components, for most wheelchair users, are below $10 \mathrm{~Hz}$. Based on maximum frequency components of $10 \mathrm{~Hz}$, the sampling rate was initially set to 20 samples/sec. To eliminate the DC component, offset and slow changes in sensor bias, the corner frequency of the high pass filter was set to $2 \mathrm{~Hz}$. The frequency response of the filter is presented in Figure 14. 

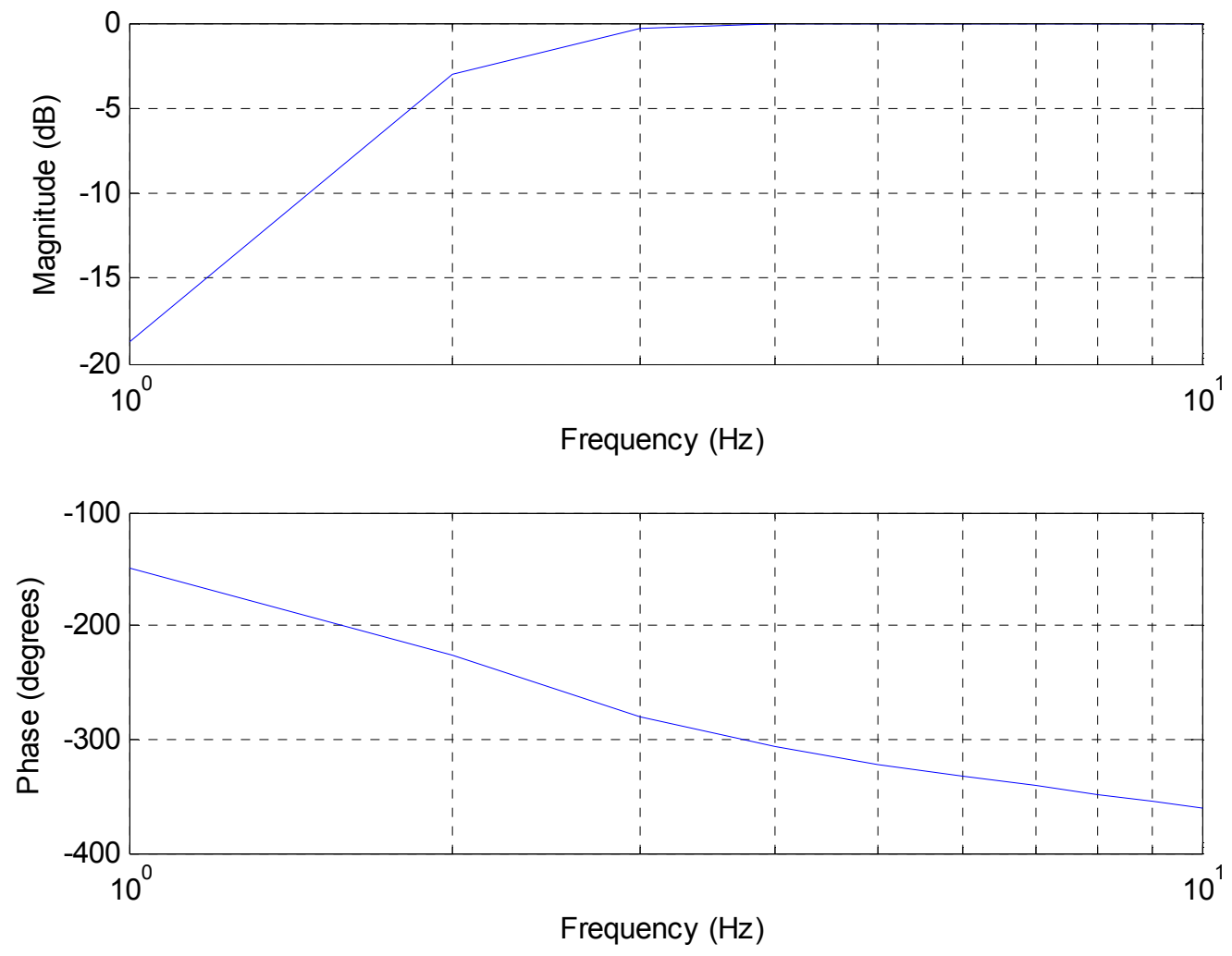

Figure 14: High Pass Digital Filter Response

The sensitivity of the system was experimentally verified as adequate to detect vibrations and any kind of motion. However, it did not interrupt storage of data when the wheelchair was static. In order to insure that the system did not discard important data, a circular buffer was maintained so that at any time a change was detected, in any of the sensors, the system stored data a half second before and at least two seconds after the event. The real time clock module of the processor was used to generate a time-stamp when a new event was detected. The basic algorithm is displayed graphically in Figure 15. 


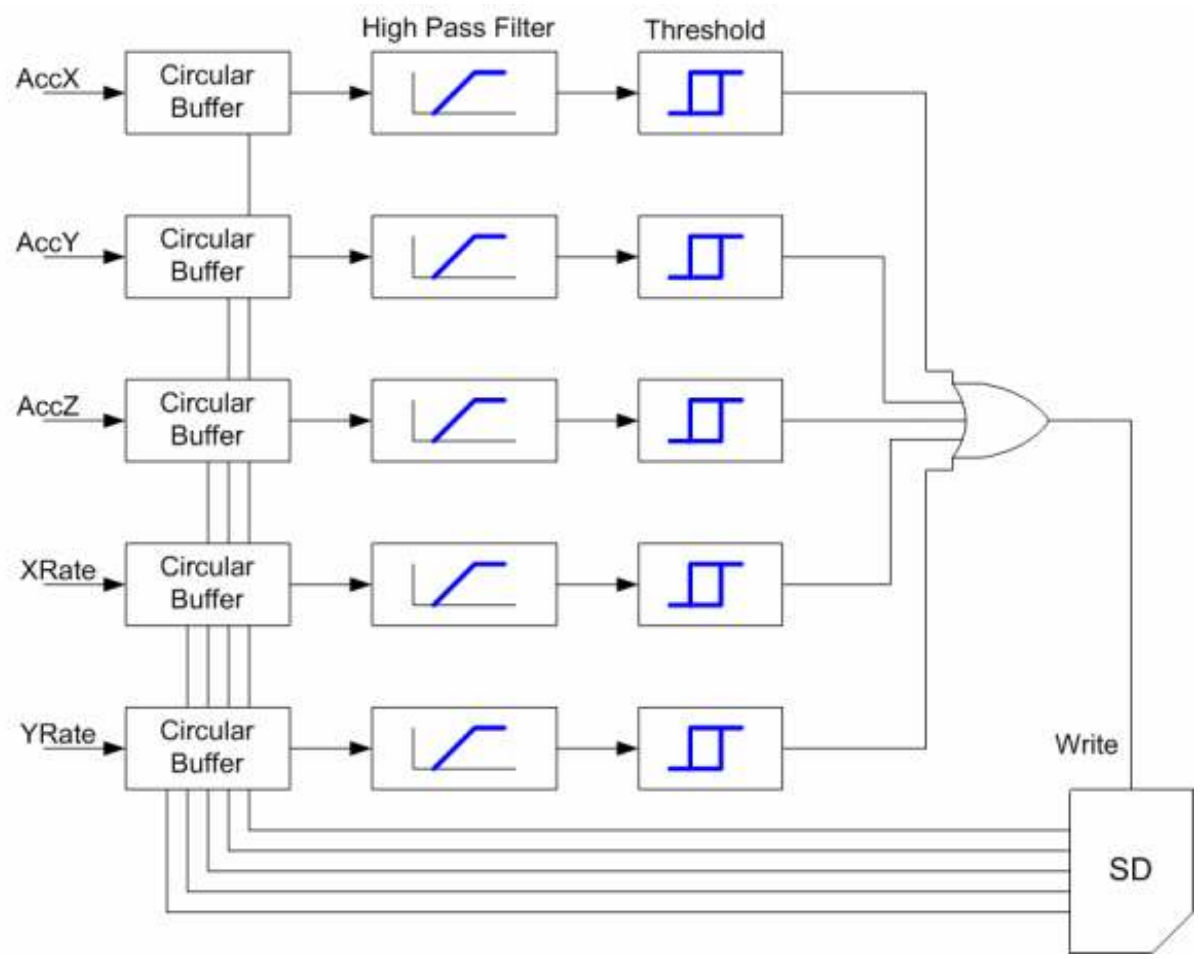

Figure 15: Real-Time Signal Processing Algorithm for Selective Data Storage

A sample of a part of one of the files generated by the embedded application is presented in Figure 16. 


$\begin{array}{lrcll}\mathrm{T} 69-602-382 & 6 / 3 / 2008-14: 10: 59 \mathrm{~T} & \\ 472 & 480 & 577 & 520 & 500 \\ 472 & 479 & 576 & 520 & 500 \\ 472 & 479 & 576 & 520 & 500 \\ 473 & 479 & 576 & 520 & 500 \\ 472 & 480 & 576 & 520 & 500 \\ 472 & 479 & 576 & 520 & 500 \\ 472 & 479 & 576 & 520 & 500 \\ 472 & 479 & 575 & 519 & 500 \\ 472 & 479 & 575 & 519 & 500 \\ 472 & 479 & 576 & 520 & 500 \\ 472 & 479 & 575 & 519 & 500 \\ 472 & 479 & 575 & 520 & 500 \\ 472 & 479 & 576 & 520 & 500 \\ 472 & 479 & 576 & 520 & 499 \\ 472 & 479 & 576 & 520 & 500 \\ 472 & 479 & 575 & 520 & 500 \\ 472 & 479 & 577 & 520 & 500 \\ 472 & 479 & 576 & 520 & 500 \\ 472 & 478 & 576 & 520 & 500 \\ 472 & 479 & 575 & 520 & 500 \\ 472 & 479 & 576 & 520 & 500 \\ 472 & 479 & 576 & 520 & 500 \\ 472 & 479 & 576 & 520 & 500 \\ 473 & 479 & 575 & 520 & 500 \\ \mathrm{~T} 69-602 & -382 & 6 / 3 / 2008-14: 21: 46 \mathrm{~T} & \\ 472 & 479 & 576 & 520 & 500 \\ 472 & 479 & 576 & 520 & 500 \\ 472 & 479 & 576 & 519 & 499 \\ 472 & 477 & 576 & 520 & 500 \\ 472 & 481 & 576 & 516 & 493 \\ 472 & 480 & 576 & 522 & 504 \\ 472 & 480 & 575 & 520 & 502 \\ 472 & 480 & 576 & 518 & 500 \\ 472 & 479 & 576 & 518 & 500 \\ 472 & 480 & 576 & 522 & 502 \\ 472 & 480 & 576 & 522 & 499 \\ 472 & 479 & 575 & 520 & 500 \\ 472 & 479 & 576 & 519 & 500 \\ 472 & 479 & 576 & 520 & 500 \\ 472 & 479 & 576 & 520 & 500 \\ 472 & 479 & 575 & 520 & 499 \\ 472 & 479 & 576 & 519 & 499 \\ 472 & 479 & 576 & 520 & 500 \\ 473 & 479 & 575 & 520 & 500 \\ 472 & 479 & 575 & 520 & 500 \\ 472 & 479 & 575 & 520 & 500 \\ 472 & 479 & 576 & 520 & 500\end{array}$

Figure 16: Sample Logged File

The first line of the frame contains, from left to right, the digitally converted values of temperature, measured by a LM35 temperature sensor, a fraction of the source voltage, a reference voltage from a precision voltage reference IC, (LM4041), with a 
constant output of 1.225 Volts and the time the new event was detected. All values are converted in the range $0-1024$ since the ADC module uses 10 bits for the conversion process of the analog signals. After the first line, which is included every time a new event is detected, the digitized sensor outputs are stored in the SD in following order:

Xrate Yrate Zacc Yacc Xacc

The final action taken towards the goal of reducing energy consumption was to reduce the clock frequency. A change from $60 \mathrm{MHz}$ to $15 \mathrm{MHz}$ produced a reduction of the average current drain from $70 \mathrm{~mA}$ to $30 \mathrm{~mA}$. The current component was only due to the microcontroller operation and processing algorithms. However, it does not include the memory writing process. The SD memory writing procedure generates an additional current burst between 12 and $18 \mathrm{~mA}$.

A Commercial-Off-The-Shelf, (COTS), rechargeable battery system was used as the source of energy for the embedded application. It was comprised of a Polymer Lithium Ion battery and a charging circuit selected for the particular battery chemistry, which was supplied by an AC wall adapter, [10], [11].

Based on analysis from the pilot study, which is presented in Section 6.1, it was concluded that the system drains an average of $1 \mathrm{~A}$ per 24 hours of normal wheelchair usage. The battery used provided 2000 mAh @ 3.7 V. Therefore, the data logger required recharging at least every other day for 7 to 8 hours since the recharging circuit provided 300 mAh@3.7 V. Additionally, even though it highly depended on user 
activity, memory usage was estimated to be approximately $20 \mathrm{MB}$ per day when sampling at 20 samples/second. Thus, sampling at 10 samples/second for daily memory usage would require approximately $10 \mathrm{MB}$. A 1 GB memory card was selected, which allows for at least two months of data collection. However, in order to more closely follow the study, it was decided that the memory card be read every month.

\subsection{Sampling Rate}

The sampling rate was initially set at 20 samples/second. The sampling rate determines the frequency, which data from the sensors is e stored in the SD card. Therefore, setting the sampling rate to an optimum value was fundamental for reducing battery consumption and memory usage. In order to evaluate the impact on the system of setting the sampling rate to 10 samples/second, two experiments were designed for assessment purposes. The method implemented consists of sampling at 20 samples/second and plotting the estimated pitch and roll angles. It is important to mention that, in accordance with the Nyquist theorem, with this sampling rate frequencies up to $10 \mathrm{~Hz}$ are completely recoverable. However, a small amount of aliasing occurred due to analog signal conditioning performed in the sensors board. The sensor board limited the sensors output frequency components to $50 \mathrm{~Hz}$. However, it was not considered to have a significant impact in human motion detection since those high frequencies are not usually included in human generated movements such as the ones carried out by wheelchair users. The above assumption was experimentally verified. 


\subsubsection{Fast Motion Experiment}

The first experiment was performed using the mechanical wheelchair pictured in

Figure 17.

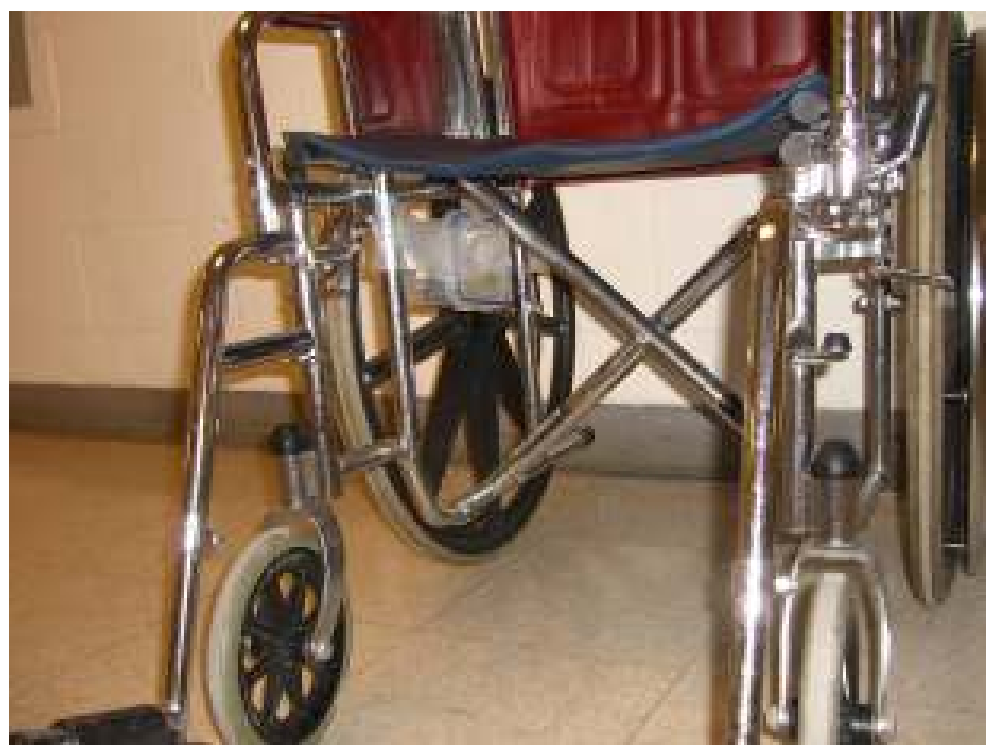

Figure 17: Mechanical Wheelchair used in the Fast Motion Experiment

In this experiment a very smooth ramp was used for the wheelchair trajectory.

The user started moving down and from side to side until reaching the end of the ramp.

This can be seen in Figure 18, which graphs the average of the pitch angle decrease in the first 20 seconds. 


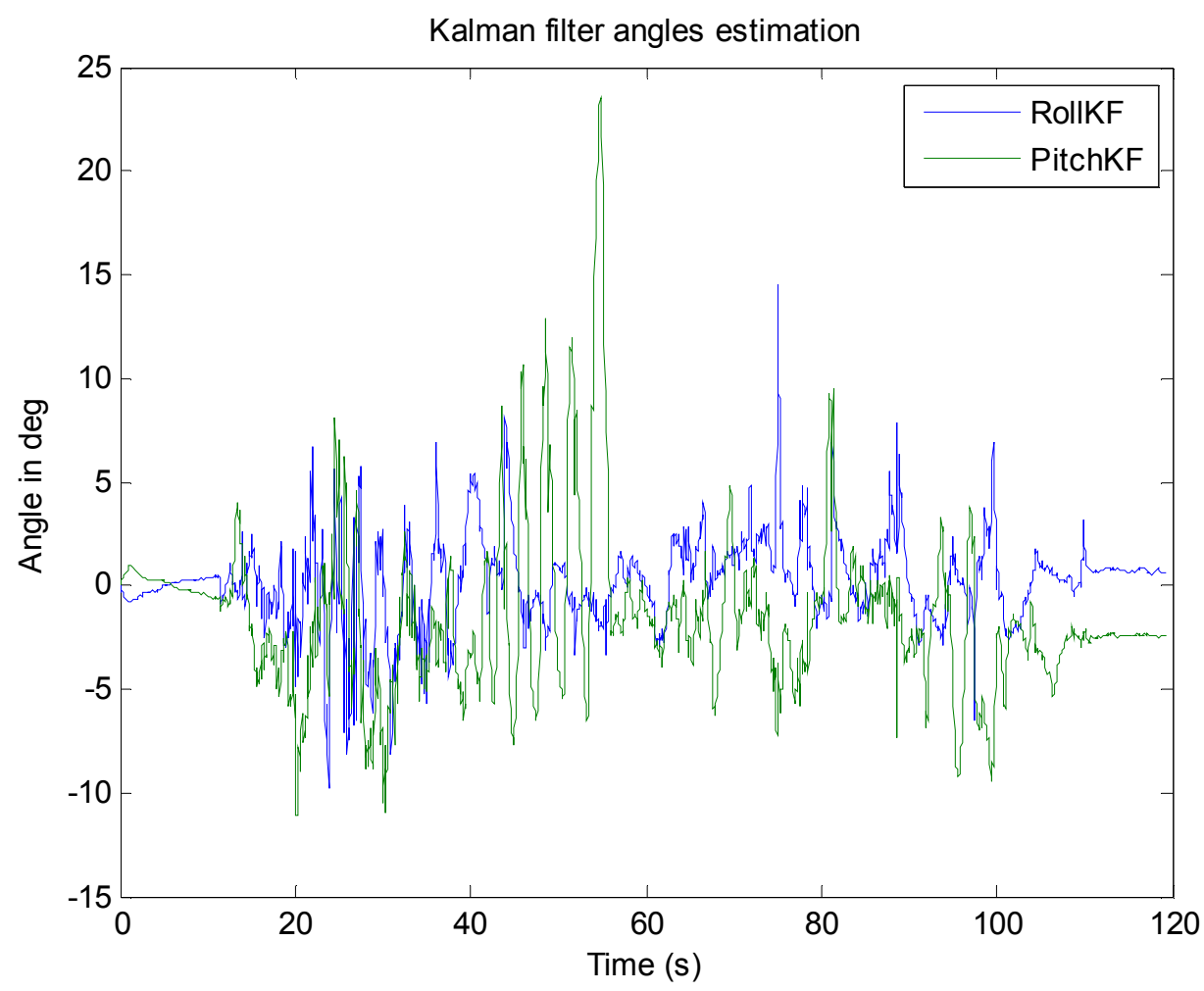

Figure 18: Estimated Angles for the Fast Motion Experiment

Afterwards, the user steered the wheelchair on its rear wheels and incremented pitch, several times. This can be clearly observed in the graph between the 40 to 60 seconds time frame of the experiment. It is interesting to notice how after each of these procedures a negative pitch angle is estimated due to the impact, which occurs when the wheelchair settles back to the ground. In order to show how the rotation towards the vertical axis negatively affects the system, the user roughly hit a wall on his right side. This can be seen in Figure 18 approximately 75 seconds after the experiment started. Figure 18, indicates that a high and sudden roll increment occurred as a result of the impact without any real wheelchair tilt. 
Figure 19 presents the spectrum of the signals generated by the gyroscopes during the fast motion experiment.
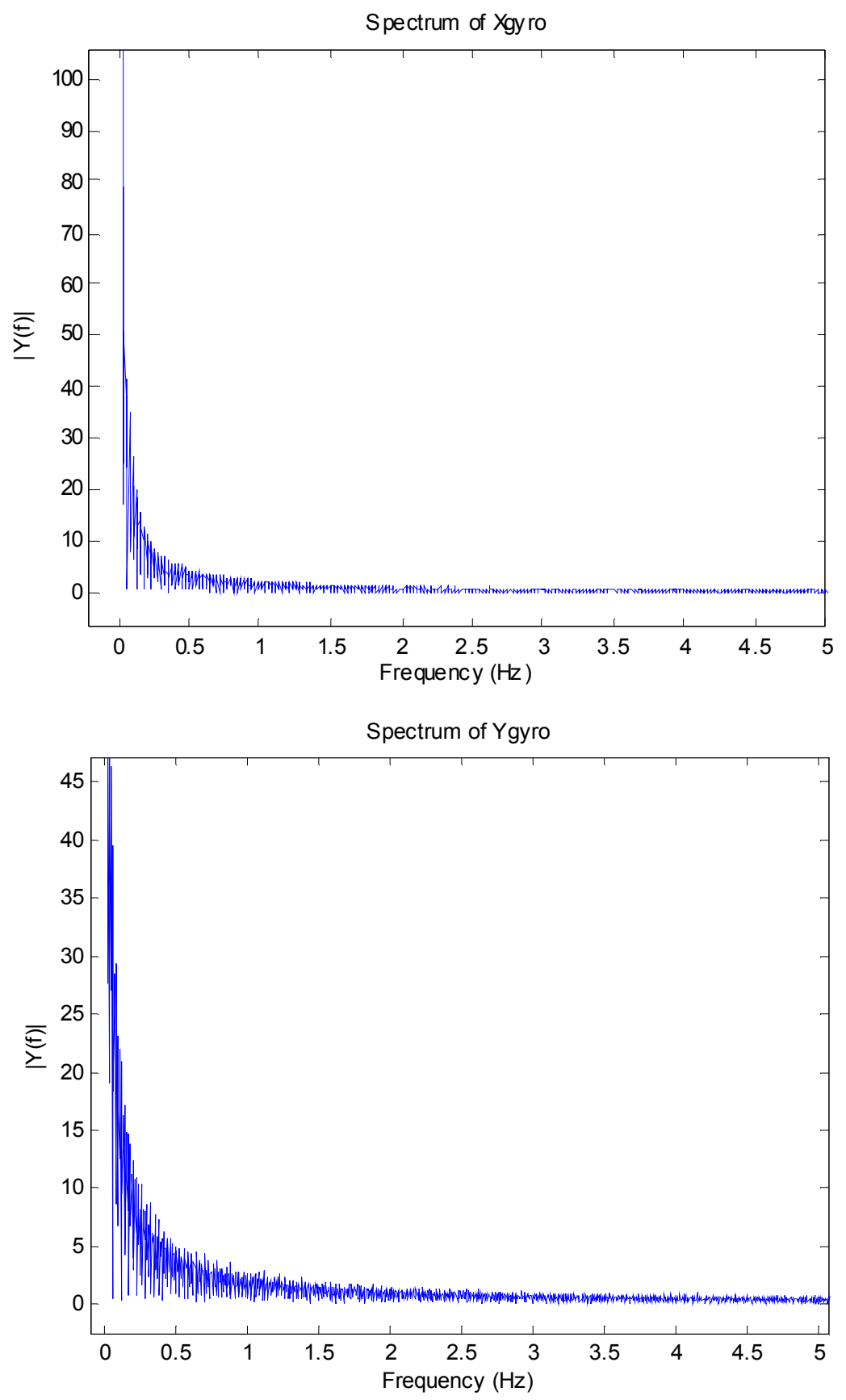

Figure 19: Gyroscopes Spectrum for the Fast Motion Experiment 
Figures 20 and 21 present the spectrum of the signals generated by the accelerometers.
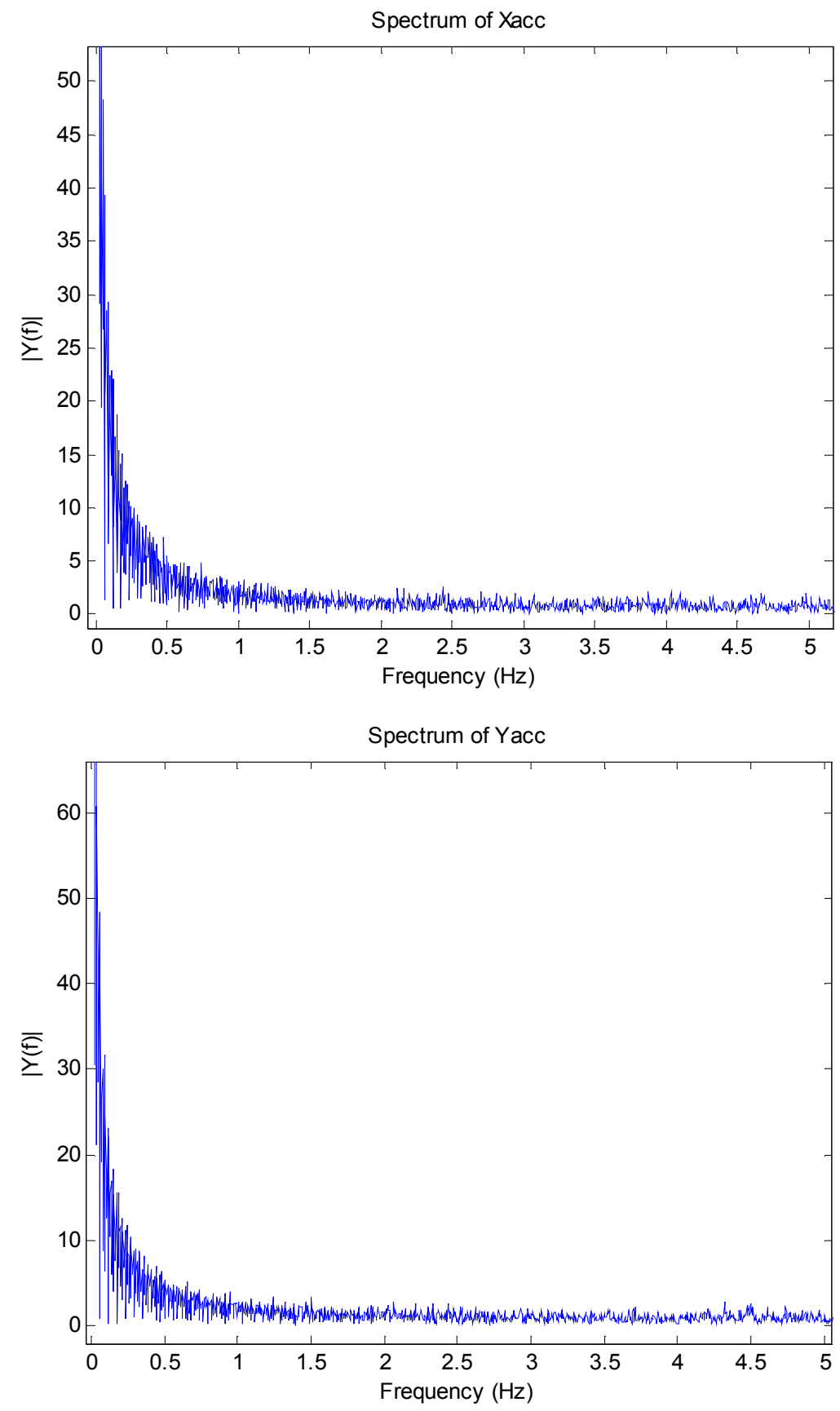

Figure 20: Spectrum of Accelerometers in the $\mathrm{X}$ and Y Directions for the Fast Motion Experiment 


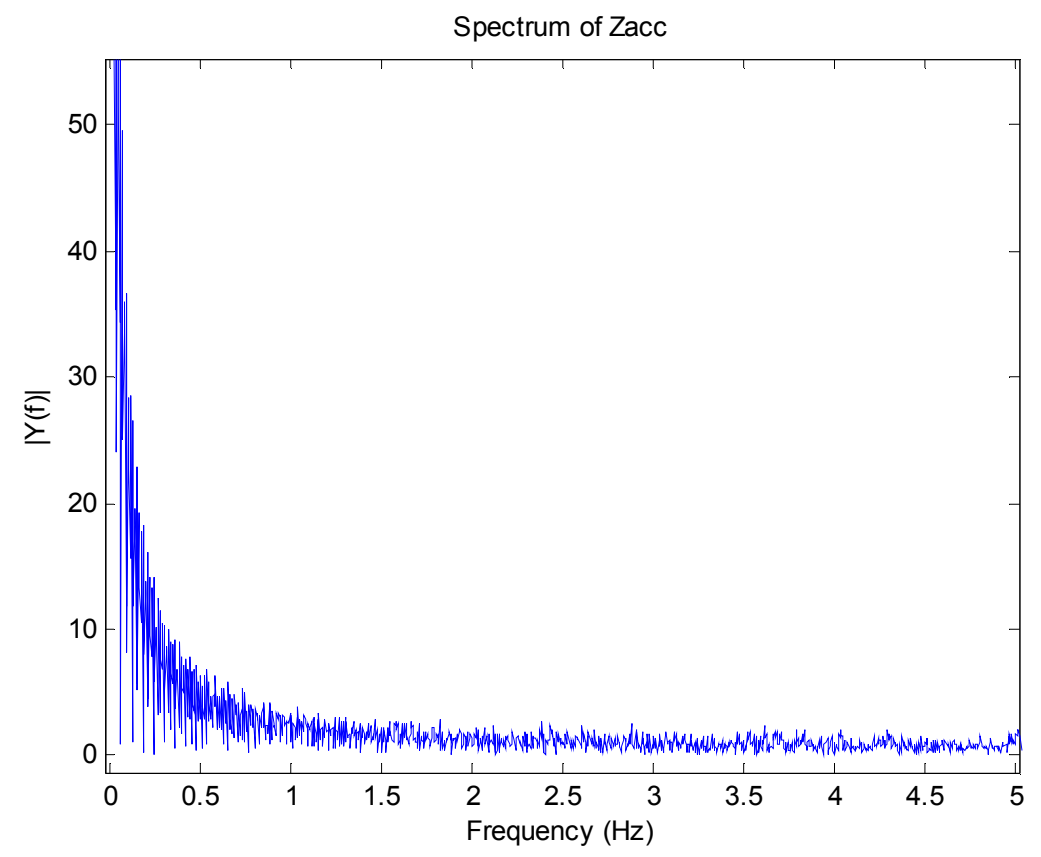

Figure 21: Spectrum of Accelerometer in the Z Direction for the Fast Motion Experiment

The gyroscope and accelerometer spectra clearly show that most of the energy is contained in the lower frequencies. A high pass filter was implemented before starting the storing process, which allowed the raw data from the different sensors including the respective time stamp information, to logged. The raw data contains all the acquired signals including the low frequency signal components.

After filtering the input signals from the accelerometers and gyroscopes values the pitch and roll angles were estimated. A low pass filter with a cutoff frequency of $5 \mathrm{~Hz}$ was utilized. Figure 22 presents the estimated angles. 


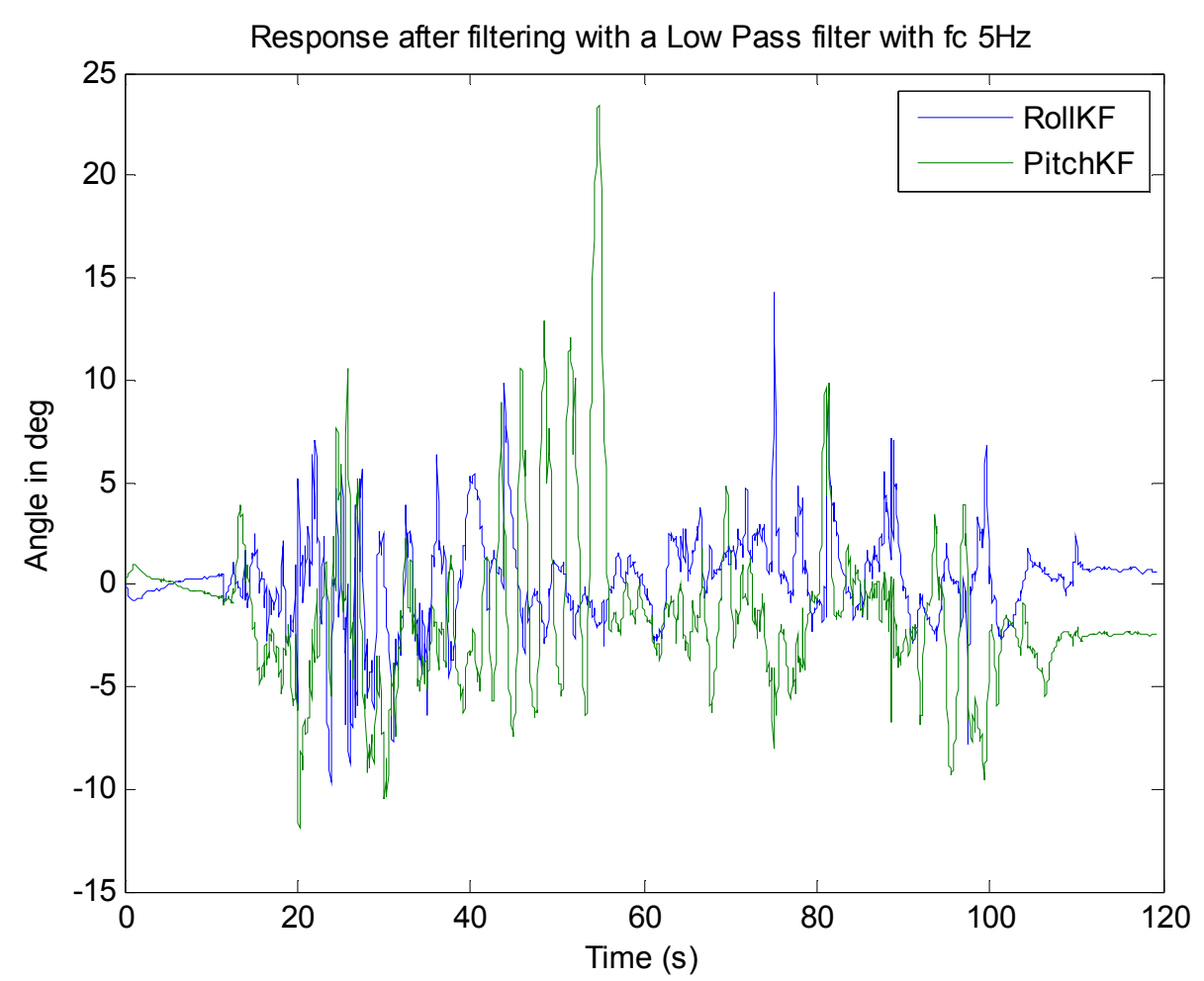

Figure 22: Estimated Angles after Filtering the Sensors Output

The spectrum of the pitch and roll angles, after filtering the sensors outputs, are displayed in Figure 23. 

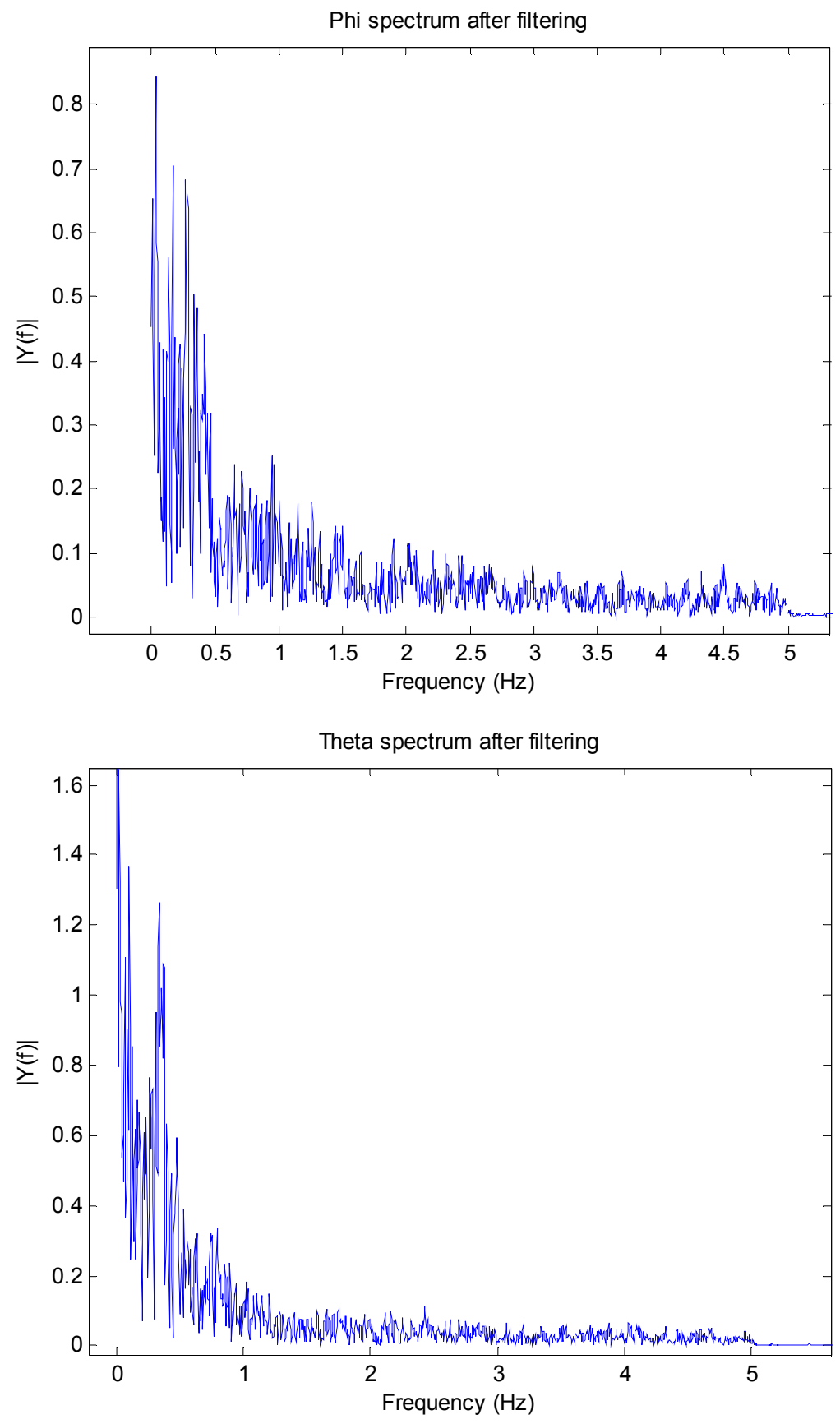

Figure 23: Pitch and Roll Spectrum after Filtering Sensors Output 


\subsubsection{Slow Motion Experiment}

This experiment was carried out using the electric wheelchair pictured in Figure

24.

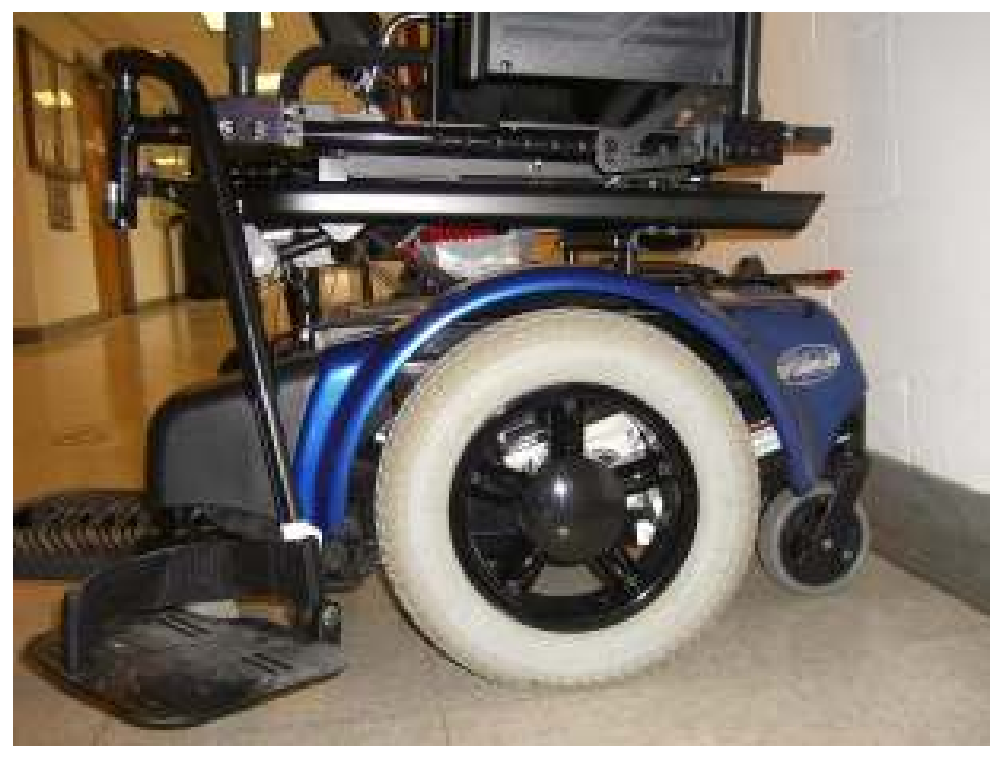

Figure 24: Electrical Wheelchair used in the Slow Motion Experiment

In this experiment, the data logger was mounted on the seat, which started to tilt upwards slowly and then came back to its original position. The tilt characterization experiment results are presented in Figure 25. 


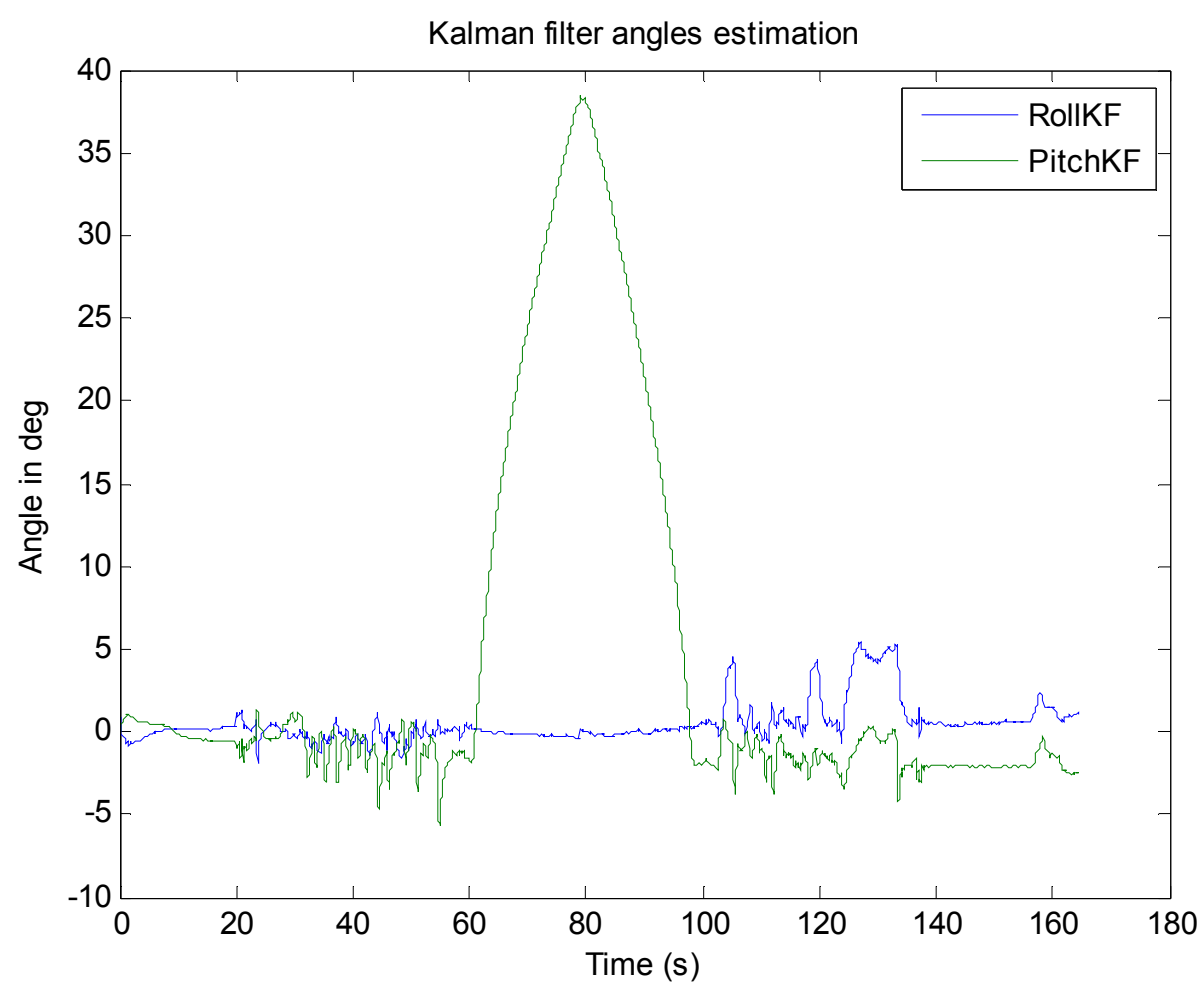

Figure 25: Estimated Angles for the Slow Motion Experiment

The tilt angle is illustrated in the graph as the pitch angle. During the interval from 125 to 135 seconds the wheelchair was continuously rotated around the vertical axis. This motion produced the same false positive, which was described in Section 4.3.1. No real inclination occurred. However, the estimated values rose to almost 5 degrees. 
Figure 26 depicts the frequency components from the gyroscopes for the slow motion experiment.
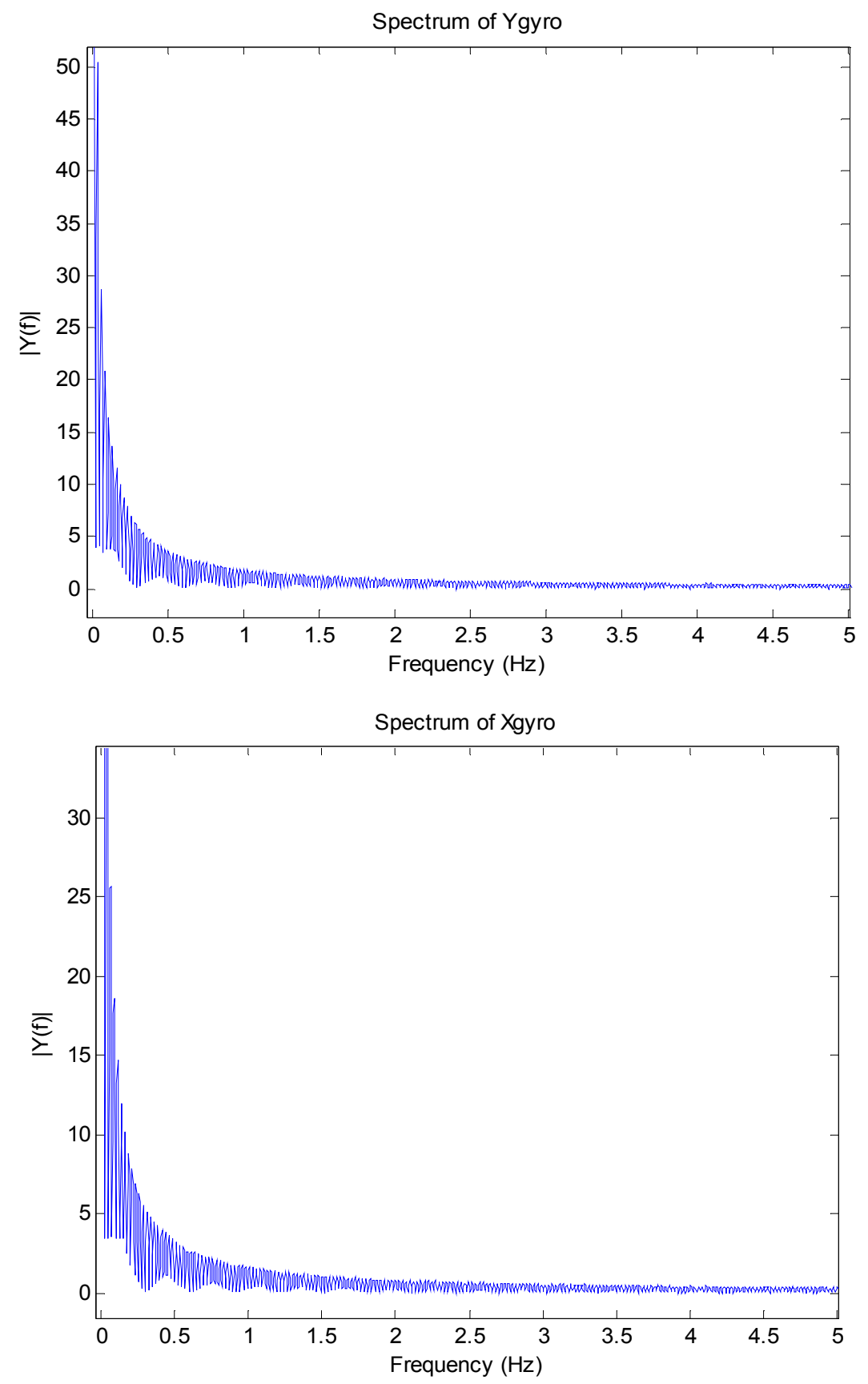

Figure 26: Gyroscopes Spectrum for the Slow Motion Experiment 
Figures 27 and 28 depict the frequency components from the accelerometers during the experiment.
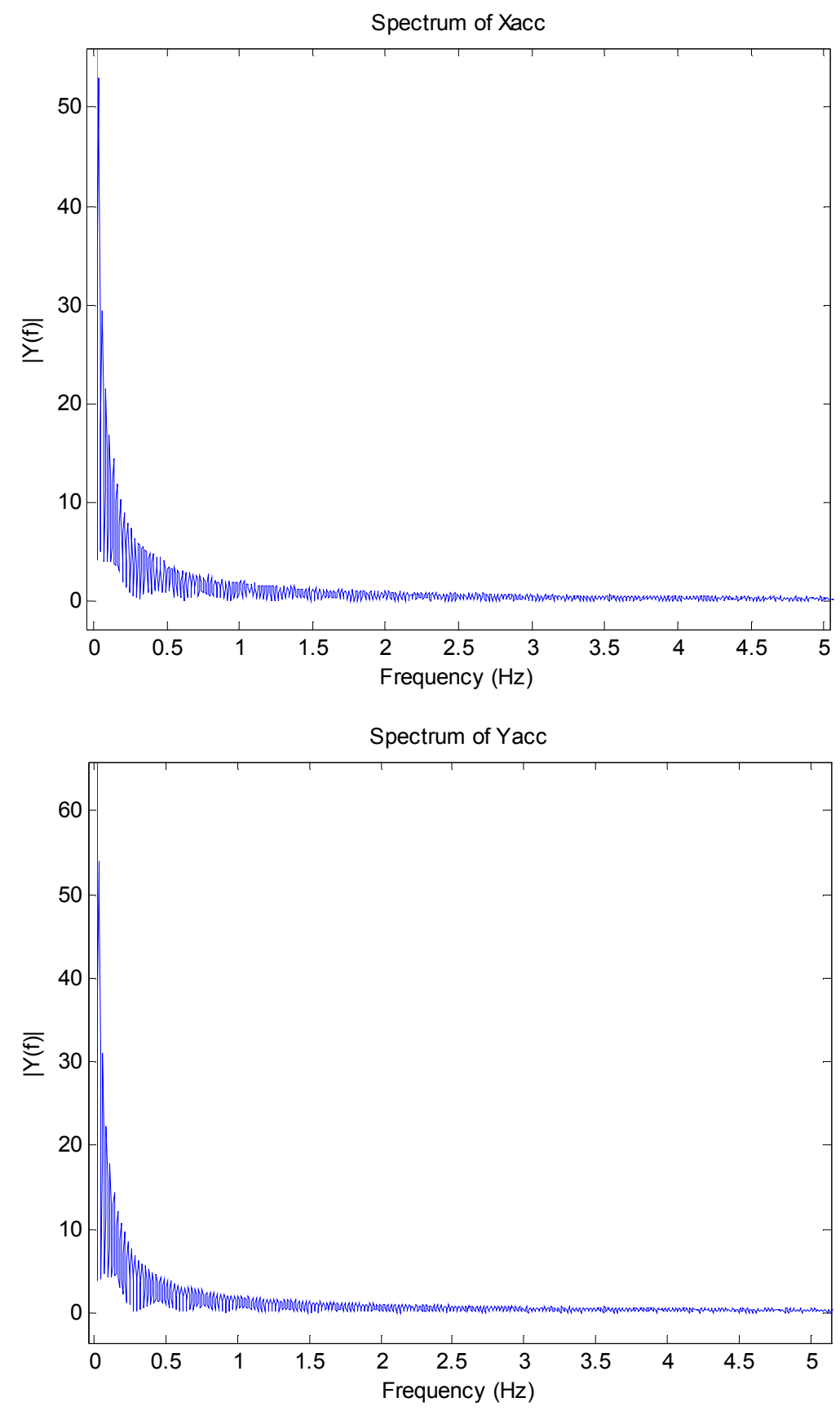

Figure 27: Spectrum of Accelerometers in the $X$ and Y Directions for the Slow Motion Experiment 


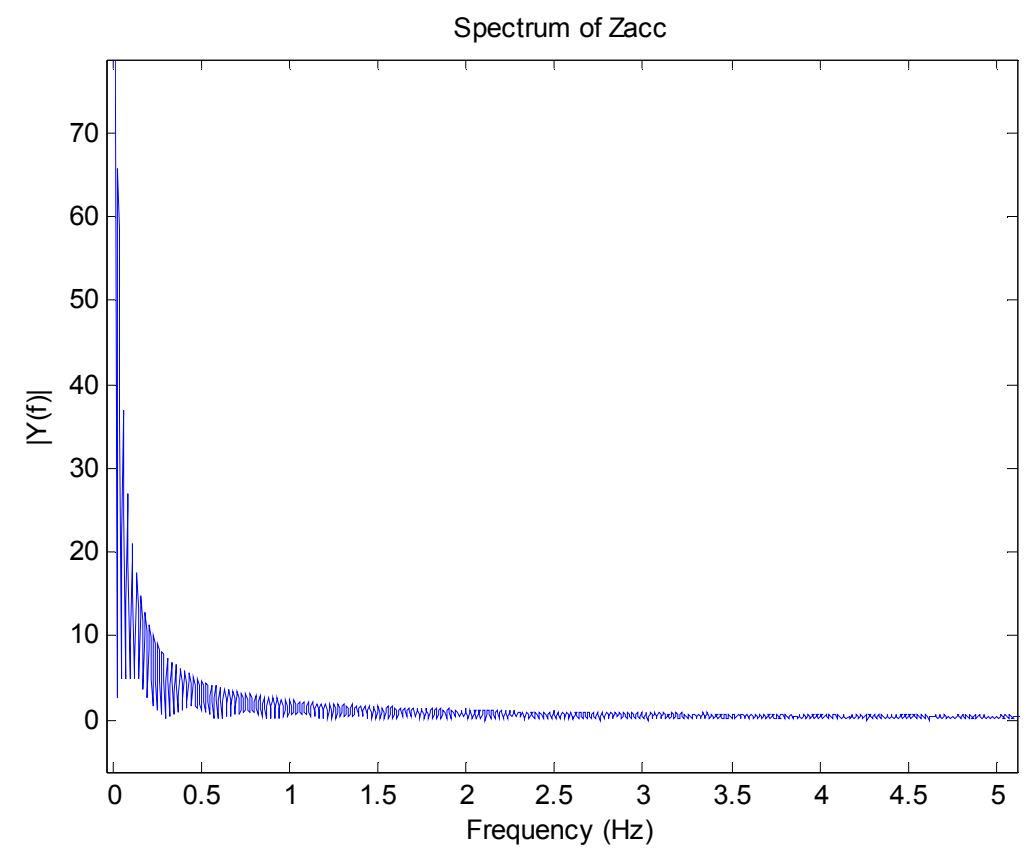

Figure 28: Spectrum of Accelerometer in the Z Direction for the Slow Motion Experiment

It is notable that the signals coming from the sensors, for the slow motion experiment, contain lower frequencies than the ones found in the fast motion experiment. This is explained by the smooth nature of the movements performed in this experiment. The sensors outputs were once again passed through a high pass filter with a $5 \mathrm{~Hz}$ cutoff frequency in order to simulate the process of sampling at 10 samples/second and evaluate the impact on performance. Figure 29 presents the spectrum for the estimated pitch and roll angles after filtering the input signals. 

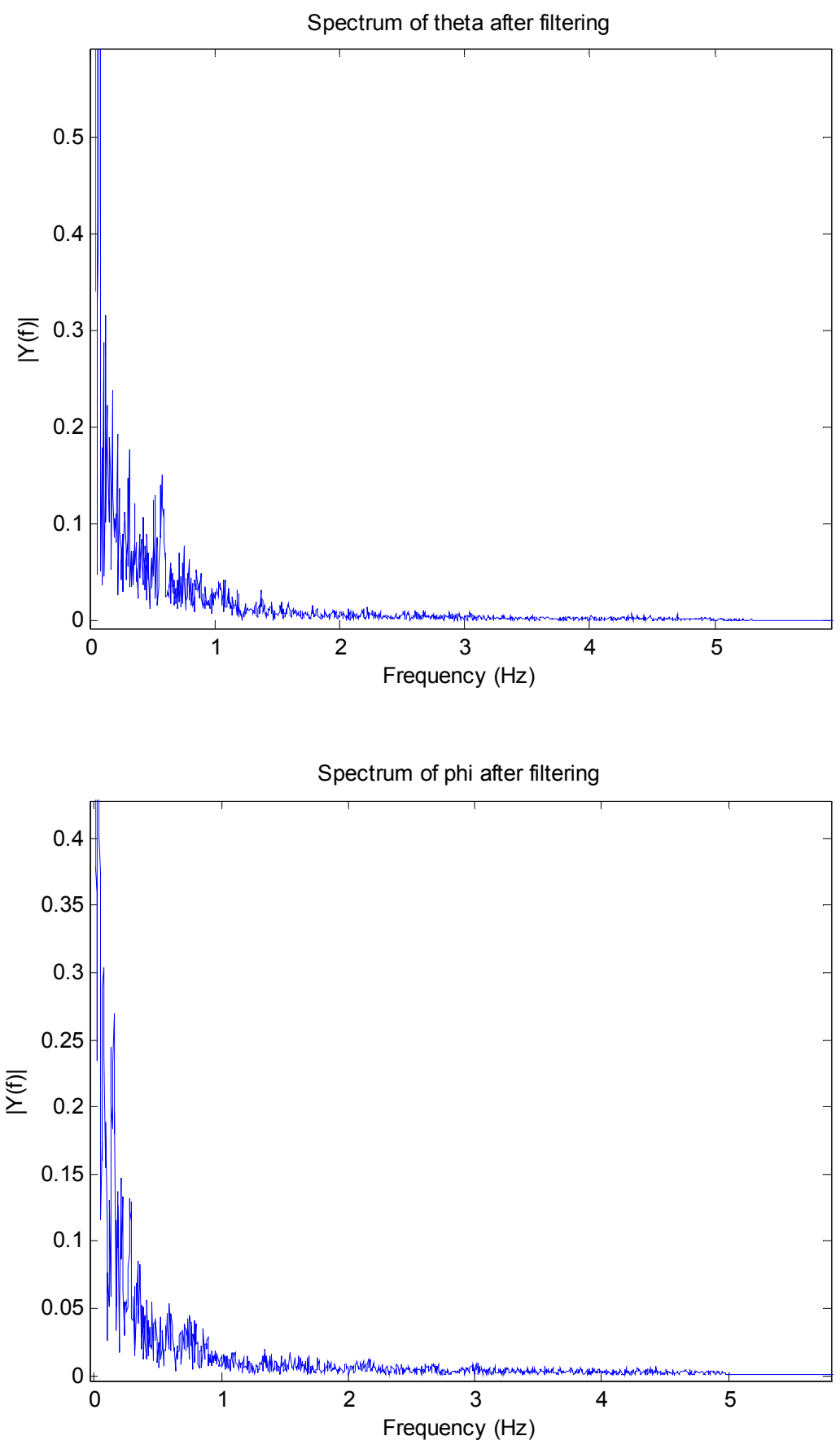

Figure 29: Estimated Pitch and Roll Spectrum after Filtering 
Figure 30 displays the estimated angles and illustrates how unnoticeable the difference is compared with the non-filtered case.

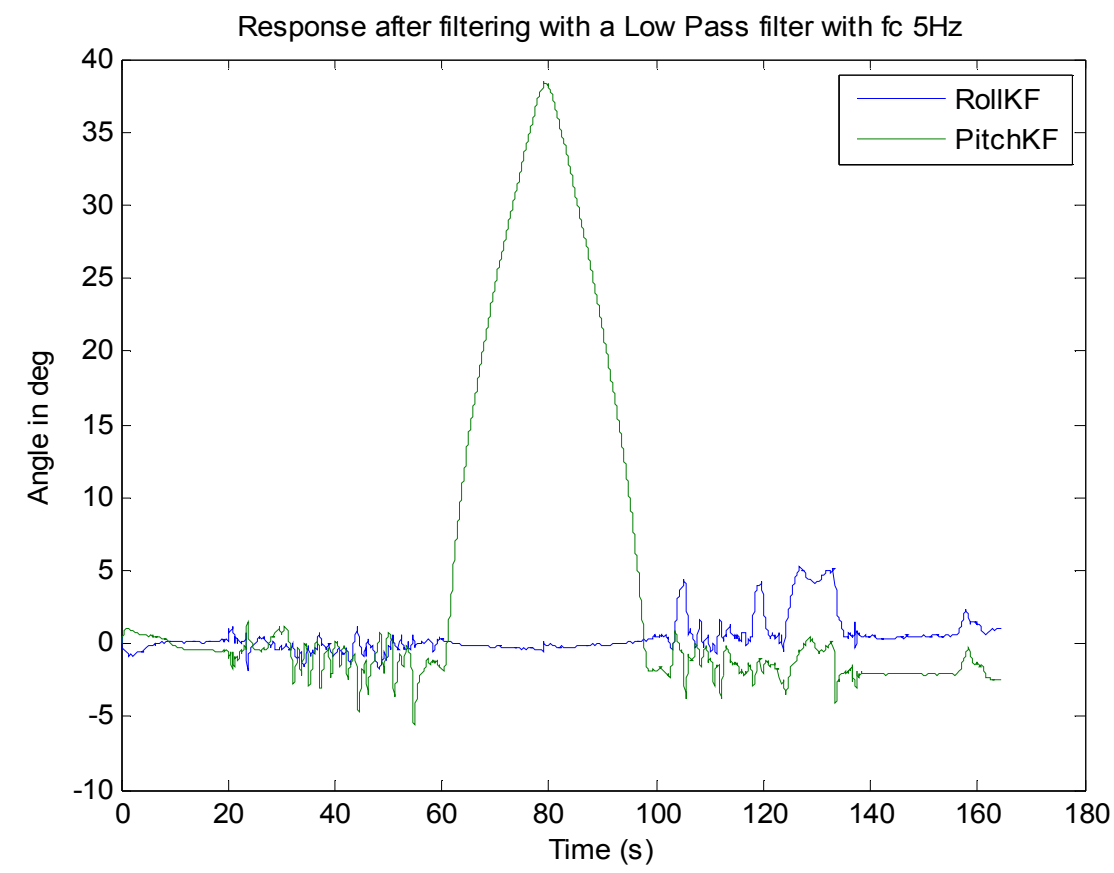

Figure 30: Estimated Angles after Filtering 


\section{Chapter 5}

\section{Sensor Fusion}

\subsection{Accelerometers Based Estimation}

Raw sensor data are stored in the SD memory for approximately one month. Afterwards, the SD card is sent to the researchers for analysis. The first approach for estimating the roll and pitch angles used only accelerometer information and assumed that the wheelchair was static. Since gravity is always sensed by the accelerometers, it is possible to calculate trigonometrically the two angles under study. Figure 31 illustrates the geometric relationships associated with the determination of the required angles.
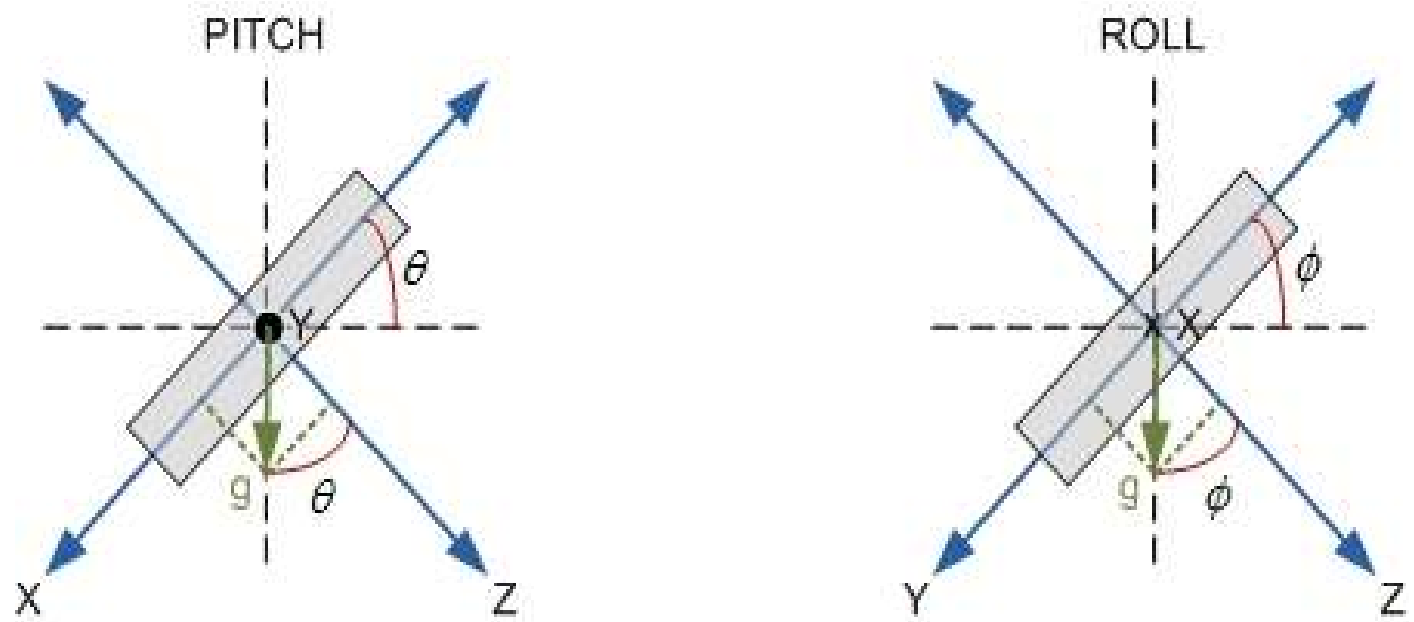

Figure 31: Gravity Components with the Wheelchair Tilted 
The mathematical relationships associated with the determination of the required angles are given by:

$$
\begin{aligned}
& \phi=\operatorname{Tan}^{-1}\left(\begin{array}{c}
\tilde{a y} \\
\frac{\tilde{a}}{\sim a}
\end{array}\right) \\
& \theta=\operatorname{Tan}^{-1}\left(\frac{\tilde{a x}}{\tilde{a z}}\right)
\end{aligned}
$$

With this simple implementation which did not incorporate gyroscope measurements, the results obtained were rather noisy.

The device could not be placed at the center of gravity of the wheelchair. Therefore, every time the user rotated around the vertical axis, an increment in the $\mathrm{Y}$ axis acceleration appeared. This motion was erroneously interpreted as a rotation around the travel direction axis, which would be roll motion. Additionally, every time the wheelchair started moving a false inclination value was obtained due to the high rate of change of the velocity in a small period of time, which created a large acceleration value.

\subsection{Kalman Filter}

The Kalman filter is an efficient recursive based methodology used to estimate the state variables of a process by minimizing the mean of the squared error, [14]. The Kalman filter can be modeled using feedback control techniques. Using these techniques 
the filter estimates the process state, which is updated with feedback signals in the form of noisy measurements. The equations for the Kalman filter model fall into two groups, which are termed time update equations and measurement update equations. The time update equations are responsible for projecting the current state and error covariance estimates, forward in time, to obtain the priori estimates for the next time step. The measurement update equations are responsible for the feedback. The feedback incorporates a new measurement into the priori estimate to obtain an improved posteriori estimate. The time update equations can also be thought of as predictor equations. The measurement update equations can be thought of as corrector equations, [14]. Indeed, the final estimation algorithm resembles that of a predictor-corrector algorithm for solving numerical problems. Figure 32 illustrates the Kalman filter process.

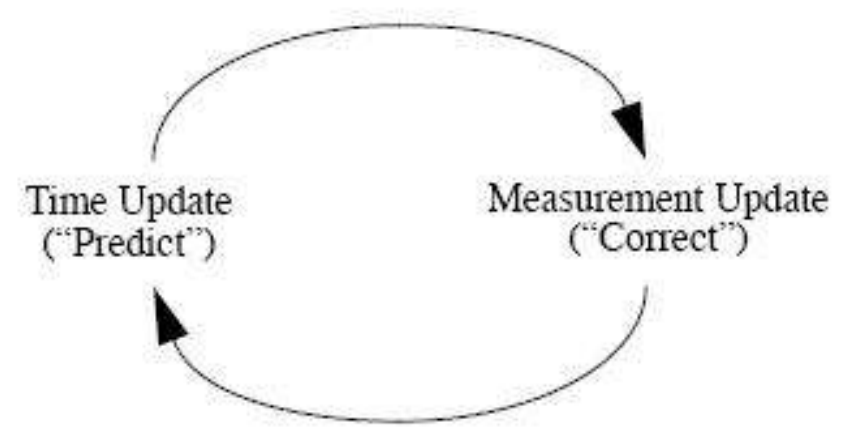

Figure 32: Kalman Filter Cycle, [14]

\subsubsection{Kalman Filter Formulation}

The Kalman filter addresses the problem of trying to estimate the state, $x$, of a discrete time process governed by a difference equation given by:

$$
x_{k}=A x_{k-1}+B u_{k-1}+w_{k-1} \text {. }
$$


The measurement variable, $z$, is given by:

$$
z_{k}=H x_{k}+v_{k}
$$

In Equation 3, the $A$ matrix relates the state at the previous time step, $k$ - 1 , to the state at the current step, $k$, in the absence of process noise. The $B$ matrix relates the optional control input, $u$, to the state, $x$. In a similar way, the $H$ matrix relates the state to the measurement, $z_{k}$. The random variables $w_{k}$ and $v_{k}$ represent the process and measurement noise respectively. They are assumed to represent white noise, with Gaussian probability distributions and are independent of each other. The probability densities of these noise variables are given by:

$$
p(w) \sim N(0, R)
$$

and

$$
p(v) \sim N(0, Q)
$$

where $N(0, R)$ and $N(0, Q)$ represent Gaussian processes with zero mean and variance $R$ and $Q$ respectively. In Equation 7 the variable $\hat{x}_{k}{ }^{-}$provides the a priori state estimate at step $k$ given knowledge of the process prior to step $k$. In Equation 8 the variable $\hat{x}_{k}$ provides the a posteriori estimate at step $k$ given measurement $z_{k}$. A priori and posteriori estimate errors were defined as: 


$$
e_{k}^{-}=x_{k}-\hat{x}_{k}^{-}
$$

and

$$
e_{k}=x_{k}-\hat{x_{k}}
$$

The priori estimate error covariance and posteriori estimate error covariance were defined by:

$$
P_{k}^{-}=E\left[e_{k}^{-} e_{k}^{-T}\right]
$$

and

$$
P_{k}=E\left\lfloor e_{k} e_{k}^{T}\right]
$$

The first goal in deriving the Kalman filter equations is to find an equation that computes an a posteriori state estimate, $x_{k}$. The variable, $x_{k}$, is a linear combination of an a priori estimate, $\hat{x}_{k}{ }^{-}$, and a weighted difference. The weighted difference is between an actual measurement $z_{k}$ and a measurement prediction $H \hat{x}_{k}{ }^{-}$. The a posteriori state estimate, $x_{k}$, is given by:

$$
\hat{x}_{k}=\hat{x}_{k}^{-}+K\left(z_{k}-H \hat{x}_{k}^{-}\right) \text {. }
$$


The difference $z_{k}-H \hat{x}_{k}{ }^{-}$is termed the measure innovation or residual and reflects the discrepancy between the predicted measurement $\hat{H}_{k}{ }^{-}$and the actual measurement $z_{k}$. A residual of zero means that the two measurements are in complete agreement. The matrix $K$ is the gain that minimizes the posteriori error covariance. This minimization is accomplished by substituting equation 11 into equation 8 , which provides $e_{k}$. Equation 8 is substituted into Equation 10, which provides the posteriori estimate error covariance. The required expectations are performed. The expectations are minimized through the derivatives, which provide the mechanism for the determination of the minimal value for $K$. The resulting $K$, which minimizes Equation 10 is given by:

$$
\begin{aligned}
K_{k} & =P_{k}^{-1} H^{T}\left(H P_{k}^{-1} H^{T}+R\right)^{-1} \\
& =\frac{P_{k}^{-1} H^{T}}{H P_{k}^{-1} H^{T}+R}
\end{aligned}
$$

Two important conclusions can be drawn from Equation 12. As the measurement error covariance, $R$, approaches zero, the gain, $K$, weights the residual more heavily since:

$$
\operatorname{Lim}_{R_{k} \rightarrow 0}\left(K_{k}\right)=H^{-1} .
$$

As the priori estimate error covariance, $P_{k}^{-1}$, approaches zero, the gain, $K$, weights the residual less heavily since: 


$$
\operatorname{Lim}_{P_{k}^{-1} \rightarrow 0}\left(K_{k}\right)=0
$$

Another interpretation for $K$ is that as the measurement error covariance $R$ approaches zero, the actual measurement, $z_{k}$, is more reliable while the predicted measurement, $H \hat{x}_{k}{ }^{-}$, is less accurate. Alternatively, as the priori estimate error covariance, $P_{k}^{-1}$, approaches zero the actual measurement, $z_{k}$, is less consistent whereas the predicted measurement, $H \hat{x}_{k}^{-}$, is more reliable.

The equations for the Kalman filter predictor-corrector cycle are depicted in Figure 33.

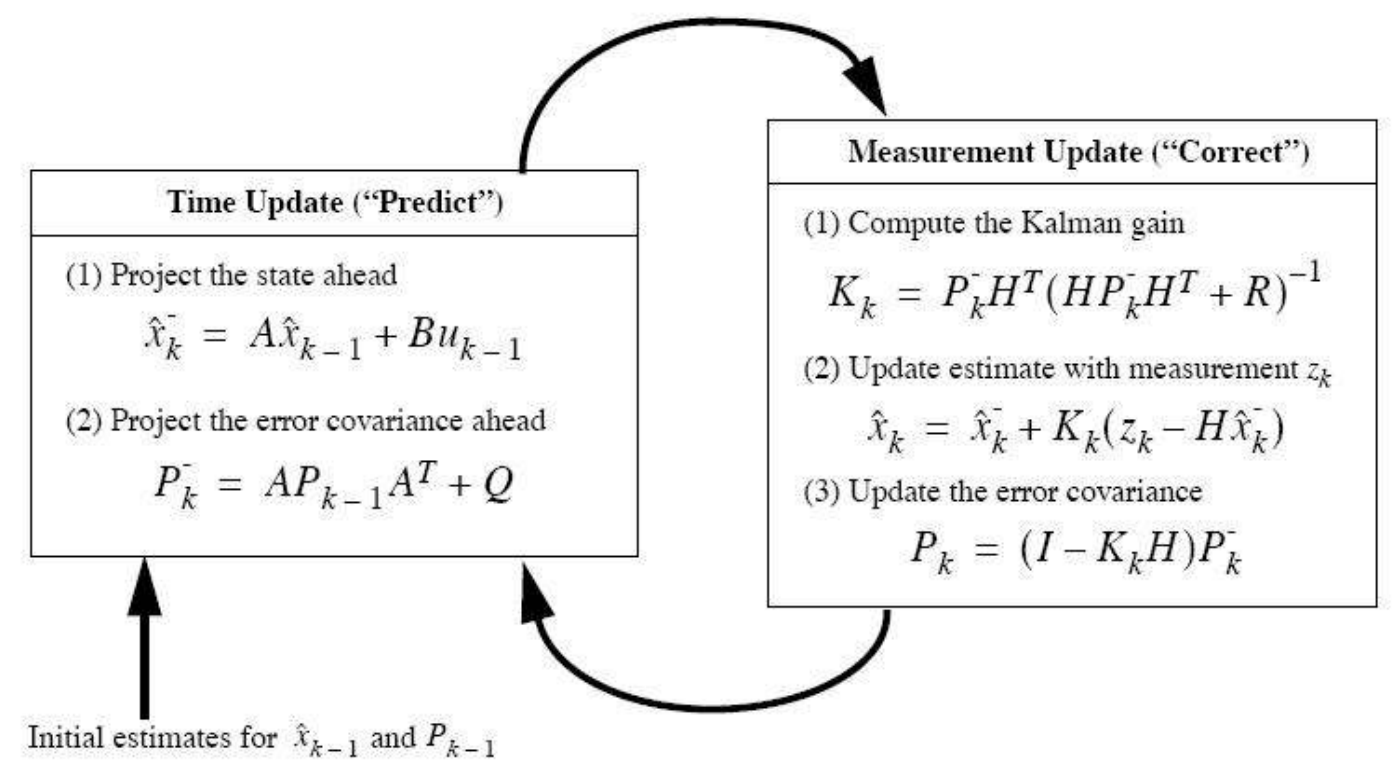

Figure 33: Kalman filter Operation, [14] 
Based on the Kalman filter formulation a block diagram describing its operation can be constructed. The block diagram is presented in Figure 34, where $r_{k}$ is the residual factor.

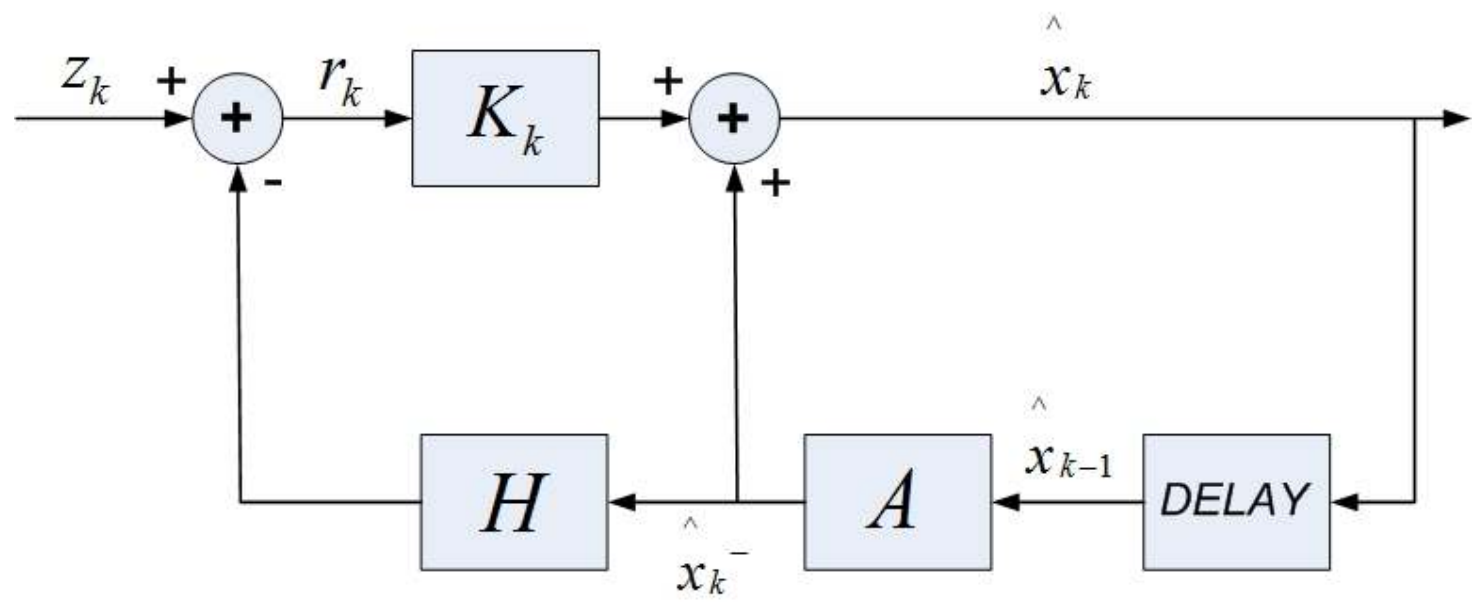

Figure 34: Kalman Filter Block Diagram

\subsubsection{Kalman Filter Implementation}

An augmented implementation approach, with respect to the approach presented in Section 5.1, was accomplished, which included the gyroscope measurements. For actual sensor fusion, a Kalman filter variant was employed to estimate the roll and pitch angles, [13].

Due to the large quantity of raw data to be analyzed, approximately 0.5 GB of data per user in one month, important considerations with regard to computational complexity need to be taken into account. Therefore, a simple and efficient variant of the Kalman filter was implemented. The equations describing the system model and the measurement model for the estimator are given by: 


$$
X_{k+1}=A X_{k}+R
$$

and

$$
z_{k}=H \tilde{X}_{k}+Q
$$

where:

- $X_{k+1}$ : Next state value calculated from the model,

- $X_{k}$ : Last estimated value for the state,

- $\quad \tilde{X}_{k+1}$ : State measured values,

- A: State transition model matrix,

- $\quad H$ : Measurement model matrix,

- $\quad z$ : Next state value from measurements,

- $\quad R$ : Model error covariance matrix,

- $\quad Q$ : Measurement error covariance matrix.

The state of the system, $\mathrm{X}$, is given by:

$$
X=\left[\begin{array}{l}
\phi \\
\theta \\
p \\
q
\end{array}\right]
$$


where:

- $\quad \phi$ : Roll angle,

- $\quad \theta$ : Pitch angle,

- $\quad p$ : Angular rate in the longitudinal direction,

- $\quad q$ : Angular rate in the lateral direction.

Pitch and roll angles were obtained indirectly from acceleration measurements.

The state transition matrix, $A$, and the measurement matrix, $H$, of the model are given by:

$$
A=\left[\begin{array}{llll}
1 & 0 & T_{s} & 0 \\
0 & 1 & 0 & T_{s} \\
0 & 0 & 1 & 0 \\
0 & 0 & 0 & 1
\end{array}\right]
$$

and

$$
H=\left[\begin{array}{llll}
1 & 0 & 0 & 0 \\
0 & 1 & 0 & 0 \\
0 & 0 & 1 & 0 \\
0 & 0 & 0 & 1
\end{array}\right]
$$

where $T_{S}$ is the sampling time, which was $100 \mathrm{msec}$. The two equations, which describe the model of the system are:

$$
\phi_{k+1}=\phi_{k}+p_{k} \cdot T_{s}
$$




$$
\theta_{k+1}=\theta_{k}+q_{k} \cdot T_{s}
$$

The equations for the estimator and the Kalman gain are given by:

$$
\begin{aligned}
& X_{k+1}=X_{k}+K_{k}\left(z_{k}-H \cdot \tilde{X}_{k+1}\right), \\
& K_{k}=P_{k} \cdot H^{T}\left(H \cdot P_{k} \cdot H^{T}+Q\right)^{-1}, \\
& P_{k+1}=\left(I-K_{k} \cdot H\right) \cdot P_{k},
\end{aligned}
$$

where:

- $P_{k}$ : Covariance matrix,

- $K_{k}$ : Kalman gain.

The Kalman filter was designed to smooth the estimation procedure since depends on both the accelerometers measurements and information from the gyroscopes. Figure 35 compares the results obtained if pitch and roll angles were directly estimated from accelerometers information only, with the results achieved after implementing the Kalman filter with both accelerometers and gyroscopes data. 

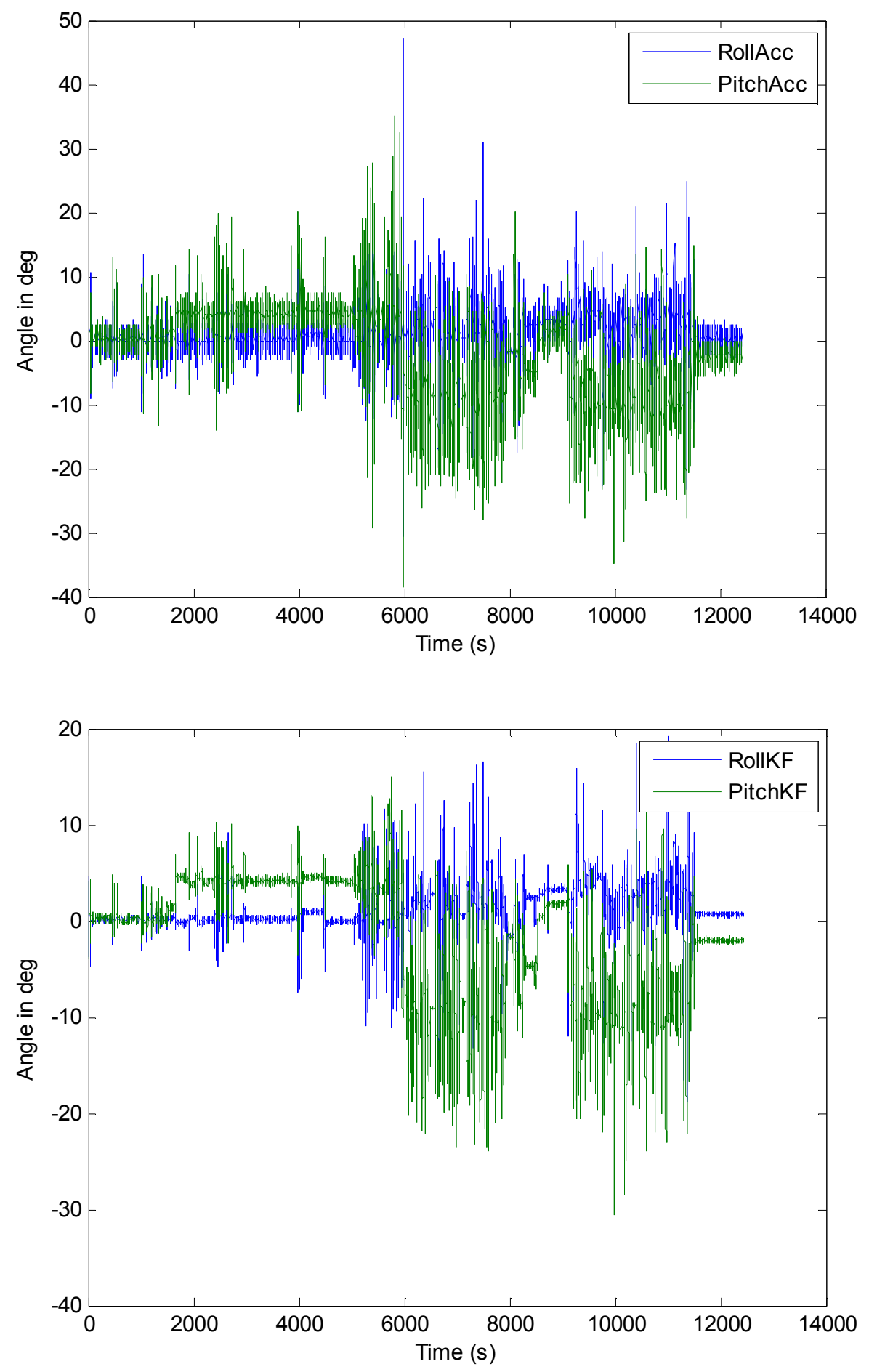

Figure 35: Results from a Three and a Half Hour Trial with a Wheelchair User 


\section{Chapter 6}

\section{Results}

\subsection{Pilot Study}

During the pilot study, the system was tested on five wheelchairs, for a period of three weeks, with the collaboration of internal subjects from the VA hospital. Feedback from actual users participating in the pilot study was very important since it provided essential information for comparison purposes and usability rating. Figure 36 pictures one of the devices attached to the side of one of the wheelchairs.

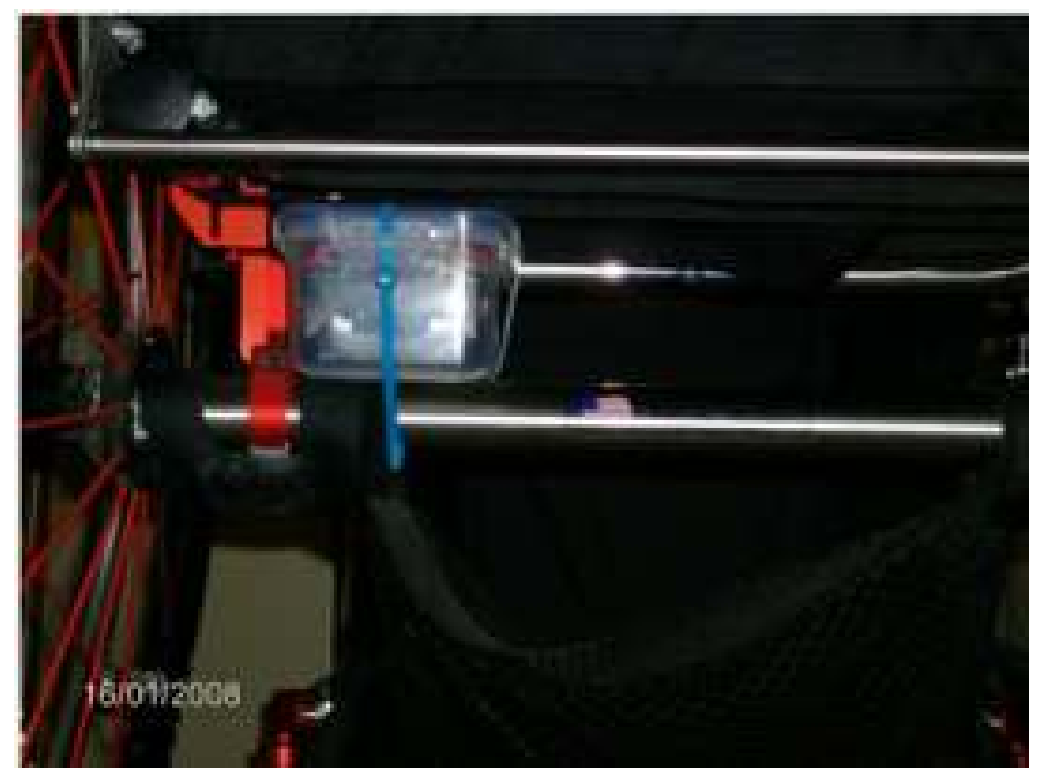

Figure 36: Device Attached to a Wheelchair during the Pilot Study 
Results extracted from the data collected for every installed system are presented by displaying graphically the estimated pitch and roll angles, as well as, a table containing the number of times each angle occurred within selected angle ranges. It is important to mention that even though some higher angles values occurred at certain times it does not mean that the user tripped or fell down. For example, the higher angle values may have been due to an impact on the device or the acceleration of a car such as when the wheelchair was being transported. In Chapter 7, some rules for tips and falls detection are described in detail, which deal with some types of false positives.

Figure 37 and Table 2 present the detailed information for subject \#1. At the beginning, the system was manipulated roughly as it was being installed on the wheelchair. Afterwards, smoother transitions took place, which represented real subject motion. 


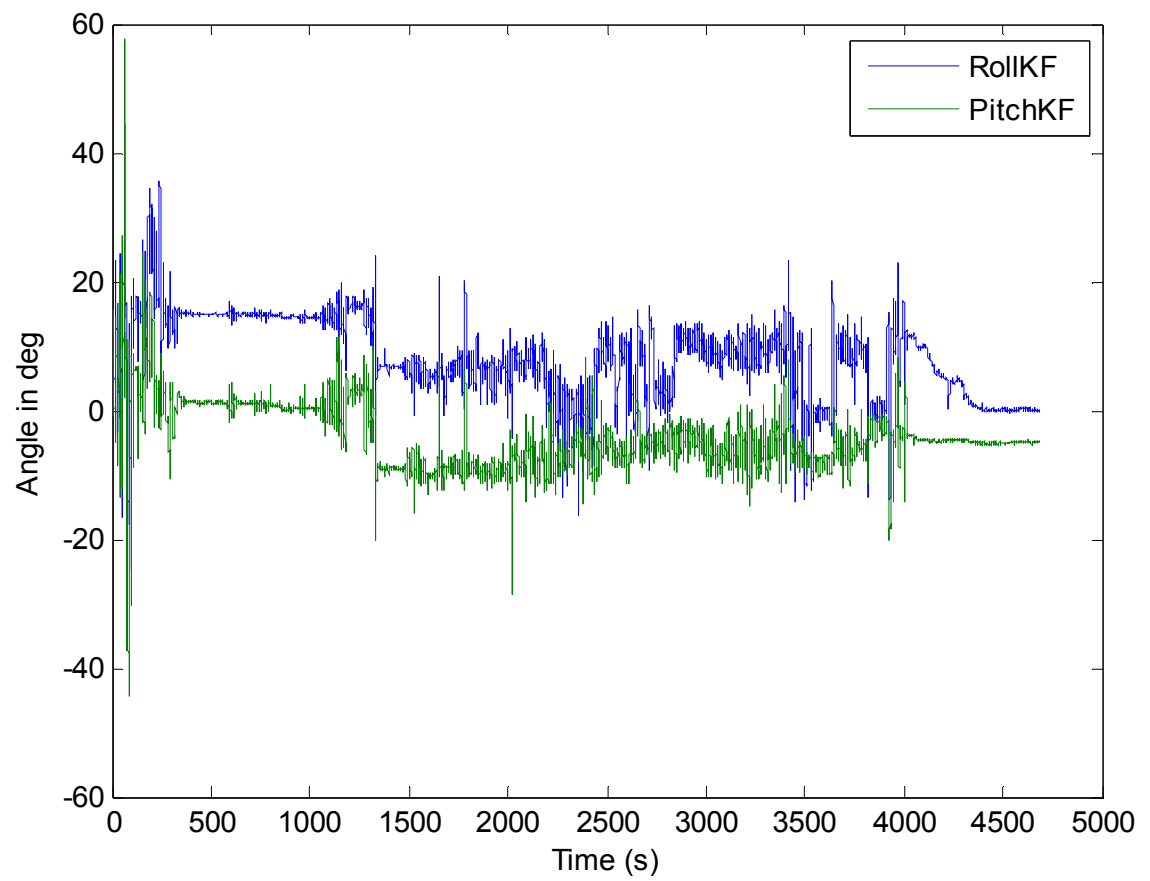

Figure 37: Estimated Angles for Subject \#1

Table 2: Angle Values Broken Down in Intervals for Subject \#1

\begin{tabular}{|c|c|c|}
\hline Angle ranges & Pitch & Roll \\
\hline $5<$ angle $<10$ & 766 & 297 \\
\hline $10<$ angle $<15$ & 483 & 266 \\
\hline $15<$ angle $<20$ & 19 & 425 \\
\hline $20<$ angle $<25$ & 3 & 23 \\
\hline $25<$ angle $<30$ & 6 & 4 \\
\hline $30<$ angle $<35$ & 1 & 3 \\
\hline $35<$ angle $<40$ & 1 & 2 \\
\hline Maximum & 57.5 & 44.4 \\
\hline
\end{tabular}


Figure 38 and Table 3 present the results for subject \#2. In the range from 6,000 to 12,000 seconds the angles were rather noisy resulting in significant changes even between simultaneous samples. Such activity does not resemble human motion. The subject verified that the data represents movement, which occurred as the wheelchair was being transported in a car.

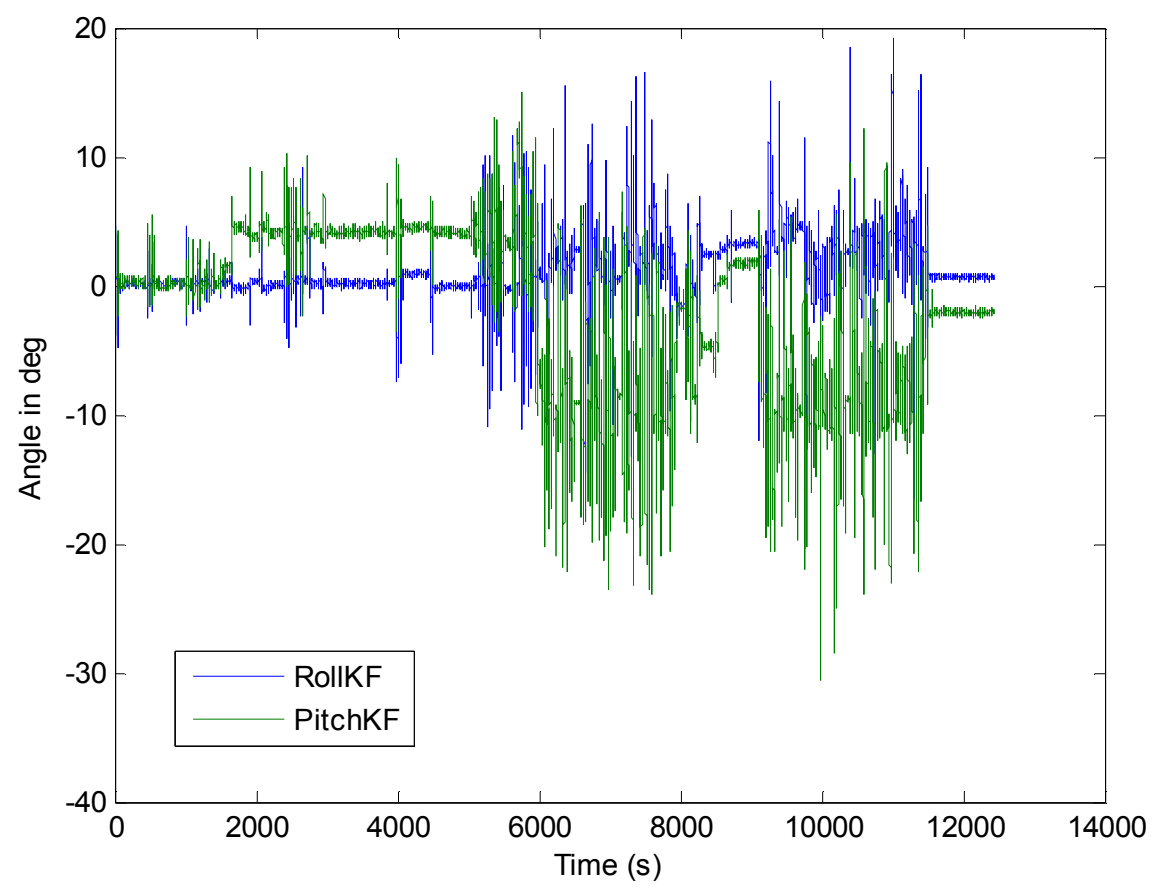

Figure 38: Estimated Angles for Subject \#2 
Table 3: Angle Values Broken Down in

Intervals for Subject \#2

\begin{tabular}{|c|c|c|}
\hline Angle ranges & Pitch & Roll \\
\hline $5<$ angle $<10$ & 43 & 305 \\
\hline $10<$ angle $<15$ & 438 & 25 \\
\hline $15<$ angle $<20$ & 77 & 14 \\
\hline $20<$ angle $<25$ & 37 & 1 \\
\hline $25<$ angle $<30$ & 1 & 0 \\
\hline $30<$ angle $<35$ & 1 & 0 \\
\hline $35<$ angle $<40$ & 0 & 0 \\
\hline Maximum & 30.5 & 20.4 \\
\hline
\end{tabular}

Figure 39 and Table 4 present the results obtained for subject \#3 during the pilot study. 


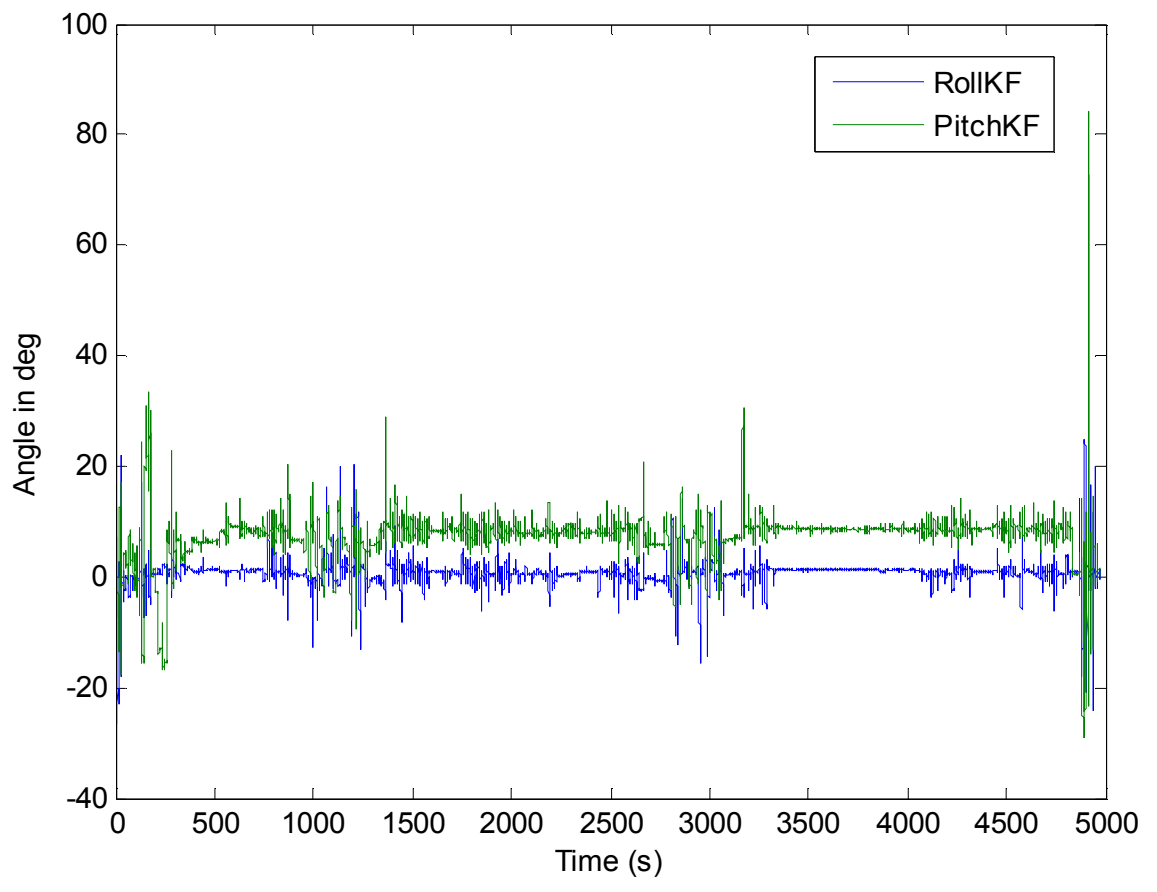

Figure 39: Estimated Angles for Subject \# 3

Table 4: Angle Values Broken Down in Intervals for Subject \# 3

\begin{tabular}{|c|c|c|}
\hline Angle ranges & Pitch & Roll \\
\hline $5<$ angle $<10$ & 358 & 48 \\
\hline $10<$ angle $<15$ & 492 & 15 \\
\hline $15<$ angle $<20$ & 16 & 12 \\
\hline $20<$ angle $<25$ & 5 & 9 \\
\hline $25<$ angle $<30$ & 1 & 4 \\
\hline $30<$ angle $<35$ & 10 & 0 \\
\hline $35<$ angle $<40$ & 0 & 0 \\
\hline Maximum & 84.1 & 72.7 \\
\hline
\end{tabular}


Figure 40 and Table 5 present the motion information obtained for subject \#4 during the study.

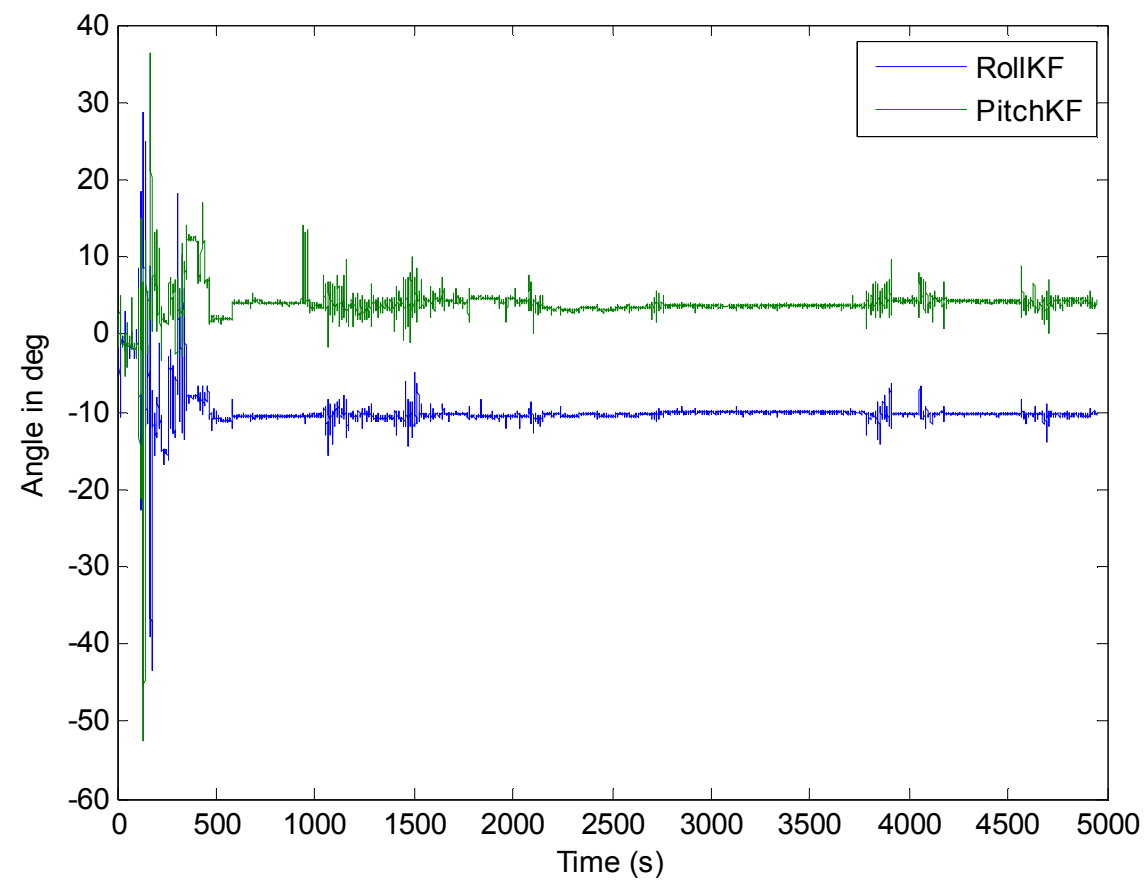

Figure 40: Estimated Angles for Subject \#4 
Table 5: Angle Values Broken Down in

\begin{tabular}{|c|c|c|}
\hline \multicolumn{2}{|c|}{ Intervals for Subject \#4 } \\
\hline Angle ranges & Pitch & Roll \\
\hline $5<$ angle $<10$ & 372 & 1090 \\
\hline $10<$ angle $<15$ & 23 & 1094 \\
\hline $15<$ angle $<20$ & 3 & 30 \\
\hline $20<$ angle $<25$ & 1 & 1 \\
\hline $25<$ angle $<30$ & 0 & 2 \\
\hline $30<$ angle $<35$ & 0 & 3 \\
\hline $35<$ angle $<40$ & 0 & 2 \\
\hline Maximum & 52.6 & 43.4 \\
\hline
\end{tabular}

The graph, in Figure 40, indicates that the subject seems to have been very stationary during the trial, especially between 2,000 and 4,000 seconds. However, two facts indicate that the data-logger may have been misaligned from its original position. The data indicate that no significant changes occurred in pitch and roll angles during the indicated stationary time and angle values stayed between approximately 4 and -10 degrees respectively.

Figure 41 and Table 6 present the results for subject \#5. The user was also stationary during some periods of time. However, the device did not move from its installation location. 


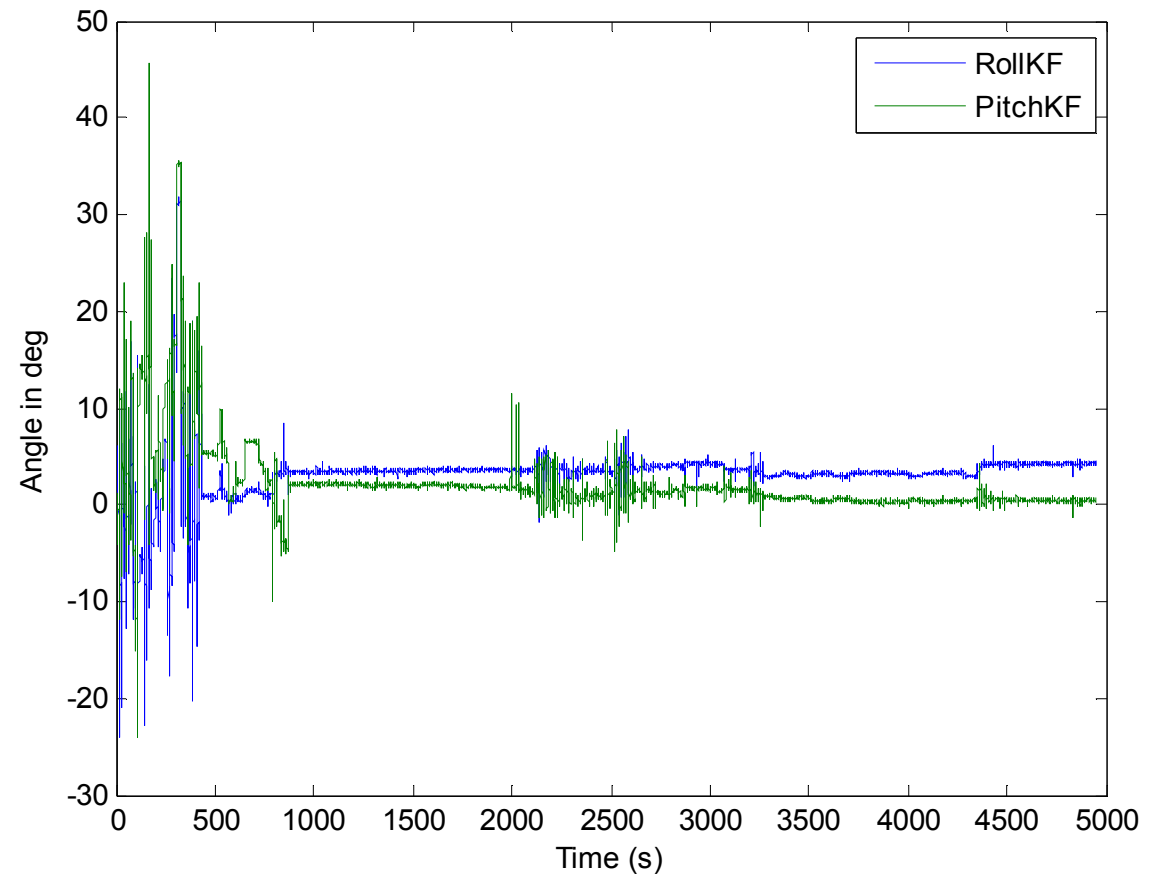

Figure 41: Estimated Angles for Subject \#5

Table 6: Angle Values Broken Down in Intervals for Subject \#5

\begin{tabular}{|c|c|c|}
\hline Angle ranges & Pitch & Roll \\
\hline $5<$ angle $<10$ & 41 & 91 \\
\hline $10<$ angle $<15$ & 20 & 29 \\
\hline $15<$ angle $<20$ & 20 & 12 \\
\hline $20<$ angle $<25$ & 9 & 6 \\
\hline $25<$ angle $<30$ & 3 & 0 \\
\hline $30<$ angle $<35$ & 1 & 1 \\
\hline $35<$ angle $<40$ & 2 & 0 \\
\hline Maximum & 45.6 & 31.7 \\
\hline
\end{tabular}




\subsection{Deployment of Wheelchair Users}

For the final deployment, 50 Motion-Loggers were assembled, tested and delivered for installation. The functionality of each device was properly verified by executing an experiment in which roll and pitch angles were forced to known positive and negative values. Thus, connections and sensors orientation were checked. The purpose of this final stage of the study was to determine tips and falls by wheelchairs users based on the estimated angles. The devices were installed and the SD cards were replaced monthly. Once the memory with the logged data arrived it was processed using the algorithm for estimating pitch and roll. The processed data was used to determine when tips of falls may have occurred. The data associated with proper data logger functionality is presented in Figure 42. 


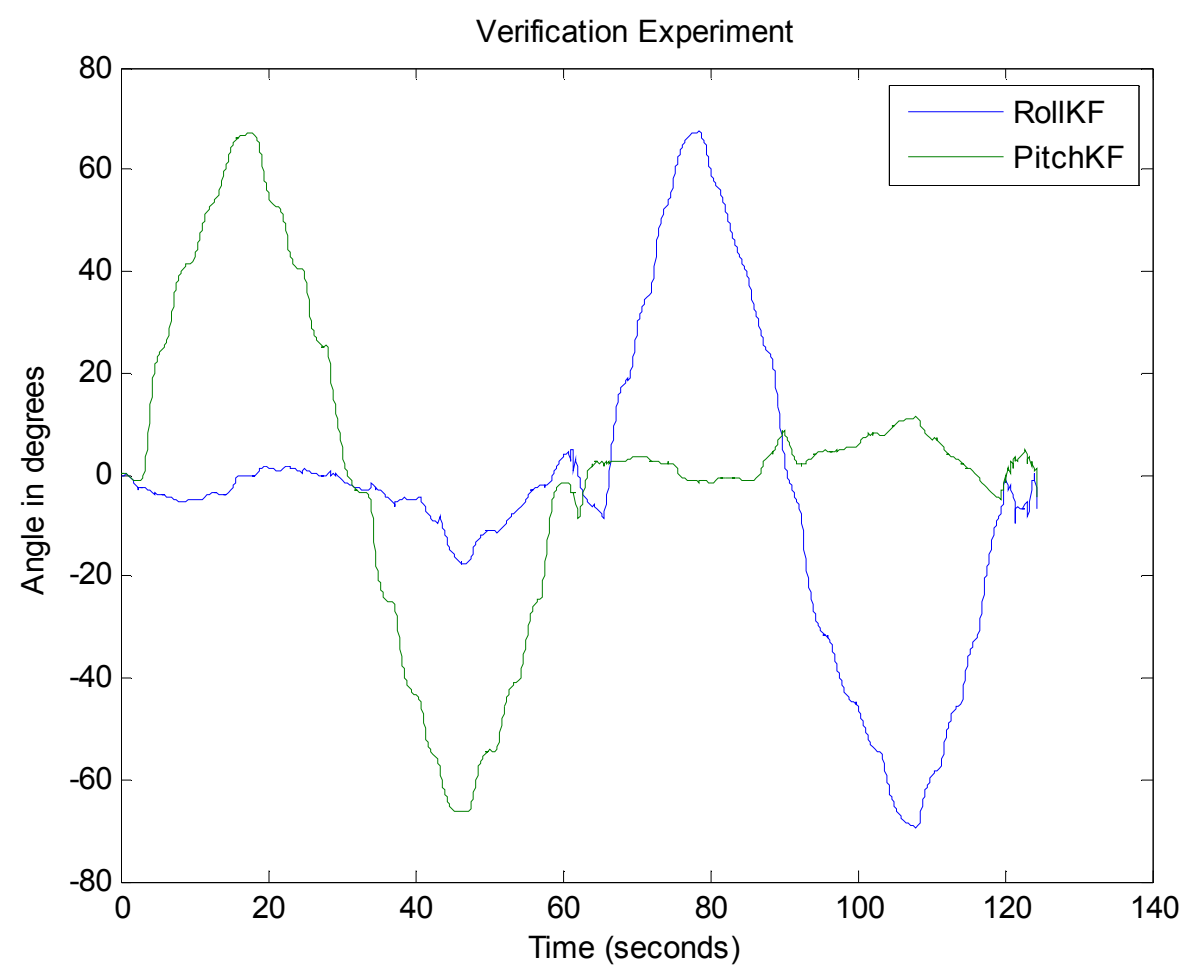

Figure 42: Experiment for Testing Proper

Functionality of the Data-Loggers

Alignment and tight coupling was crucial for the accuracy of the results.

Otherwise, the sensors might possess a biased inclination angle and/or measure the vibration of the device relative to the wheelchair. Figure 43 pictures two of the wheelchairs with the attached devices. 

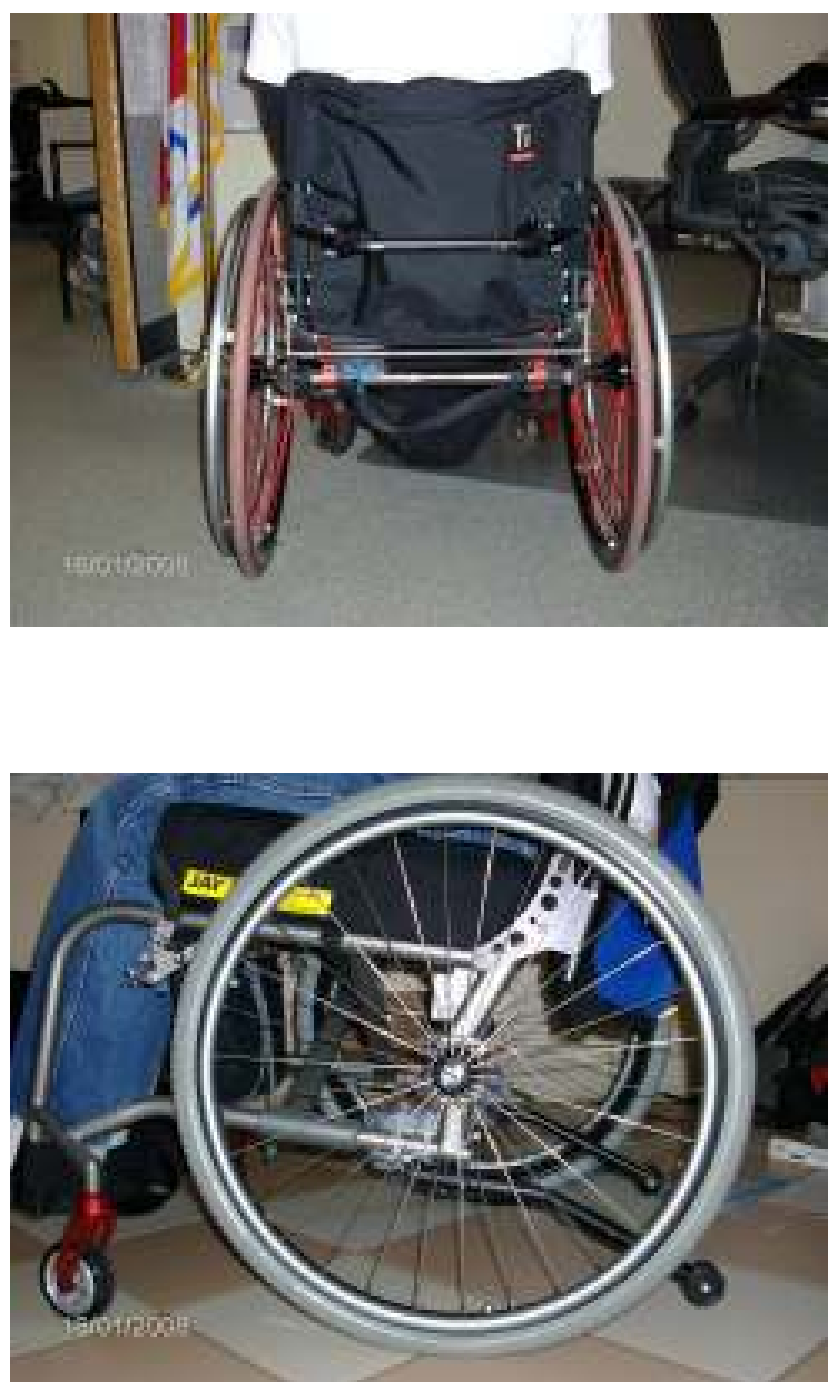

Figure 43: Data Logger Attached to a Wheelchair During the Final Deployment Stage

After processing the data in MATLAB, a spreadsheet file was generated within which the subject number, the total number of tips, the total number of falls and a timestamp representing the date and time when each event occurred are displayed. Figure 44 presents a picture of the spreadsheet generated for subjects 4,8 and 17 . 


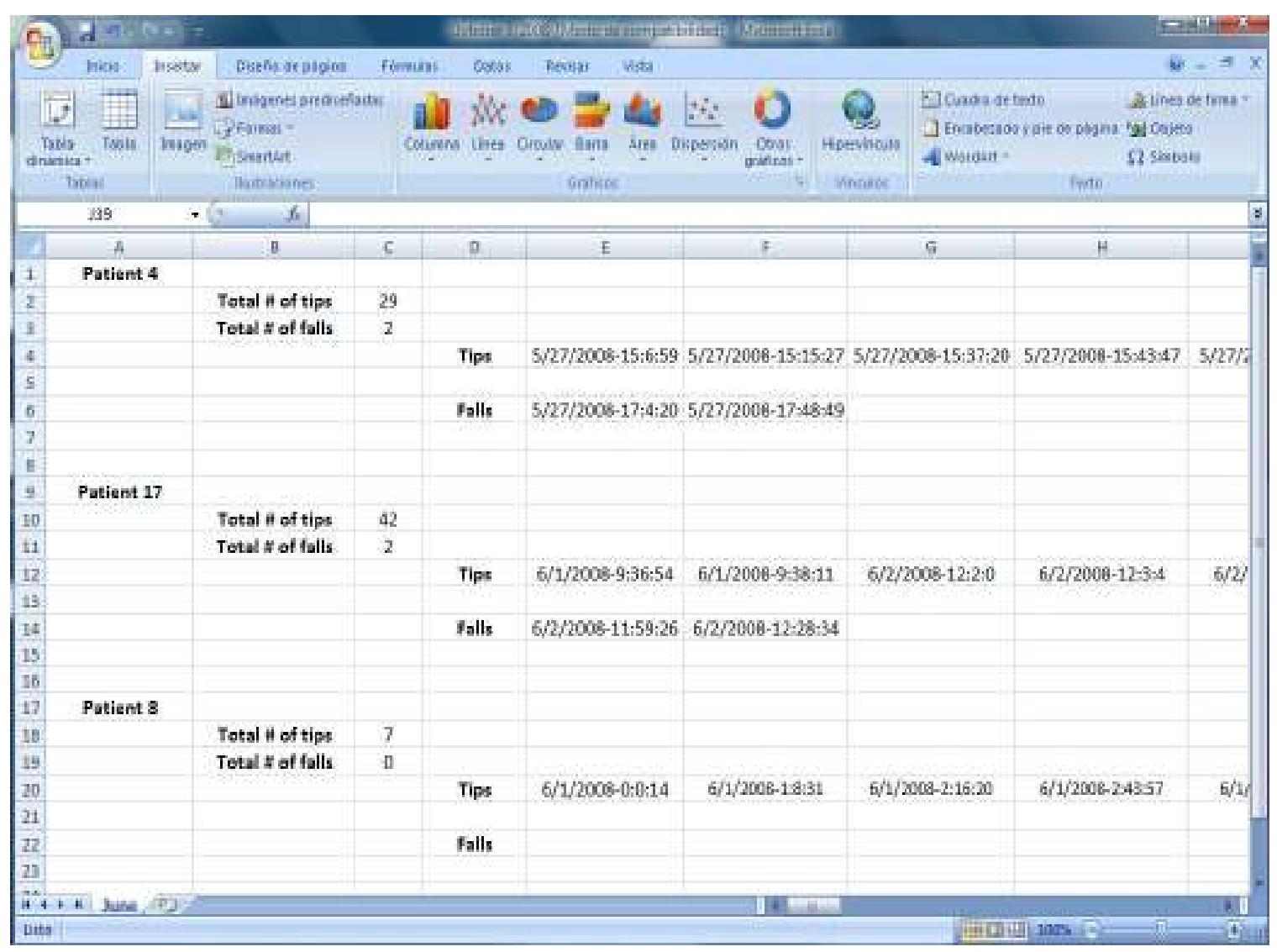

Figure 44: Spreadsheet Displaying the Number of Tips, Falls and Time-Stamps

After obtaining the estimated longitudinal roll and lateral pitch angles, a processing algorithm, which followed certain specific rules was executed for falls and tips detection. The algorithm steps were:

- The algorithm checked that the battery was properly charged at the time the tip or fall was detected, which indicated sensor measurements were reliable.

- At the beginning of each frame, whose length depended on the activity intensity of the user after the commencement of motion, the software 
checked that the first estimated angles did not contain high values. This assisted in the avoidance of the false detection of an event whenever the wheelchair was stationary and tilted without the subject being onboard. This situation could happen when the system stopped logging data when motion was undetected, even with the wheelchair tilted.

- If any of the angles were higher than the threshold, during a certain amount of samples, the proper event was detected. The thresholds were set to $20^{0}$ for tips and $30^{\circ}$ for falls. The algorithm also checked that the angle was not changing its polarity between contiguous samples since such indication could represent the case of the wheelchair being transported in a vehicle as depicted in Figure 38. Human motion is not likely to generate fast tilt changes.

- The software developed a value by adding the differences of the current sample and the 5 previous samples. If the value was higher than a threshold, a fall event may have happened and was indicated as a sudden rate of change in the estimated angles.

Figures 45 and 46 display 4 graphs containing the variation of angles during a frame when a tip was detected for subject \#4. 

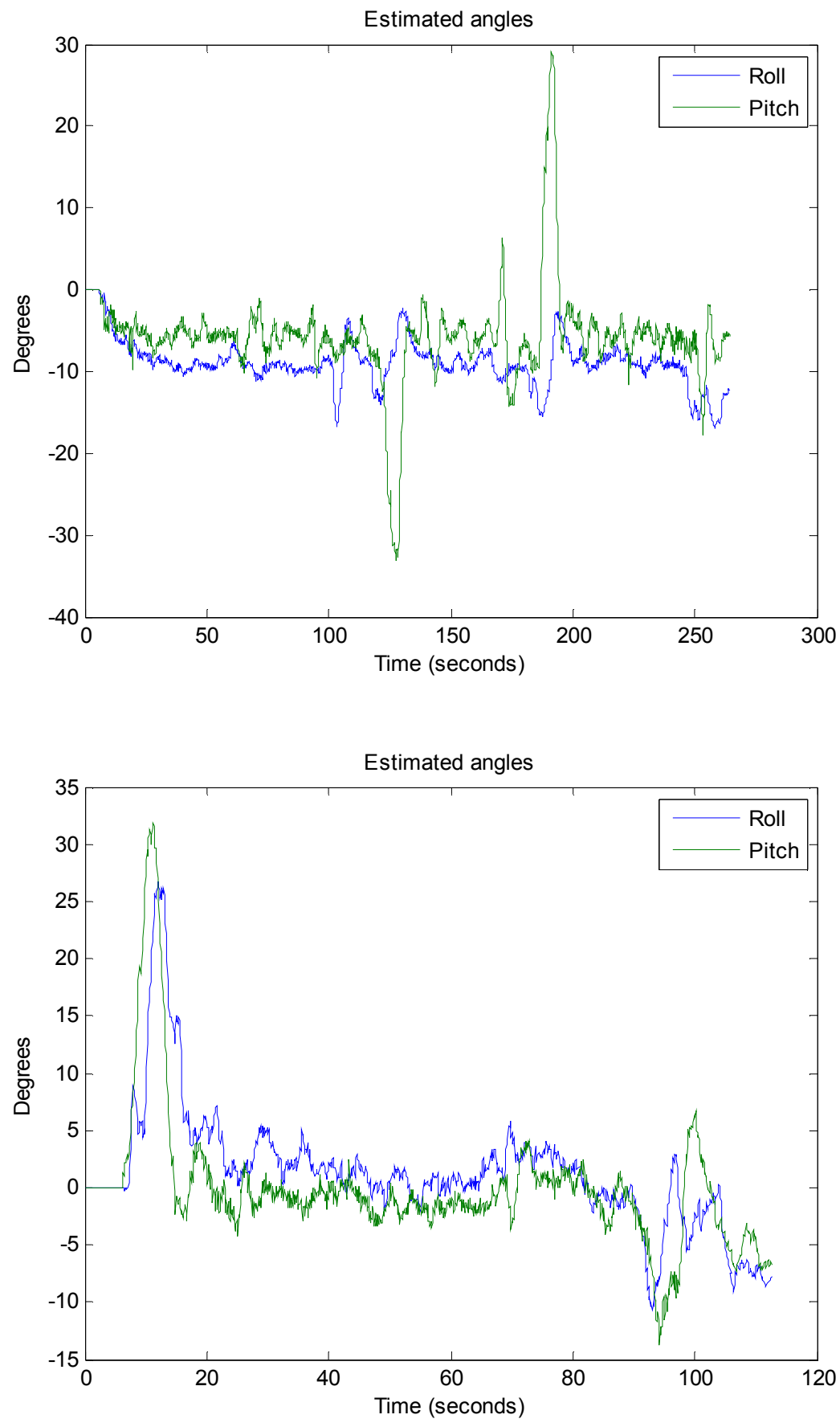

Figure 45: Pitch and Roll Variations during Tip

Detection in Frames 1 and 2 for Subject \#4 

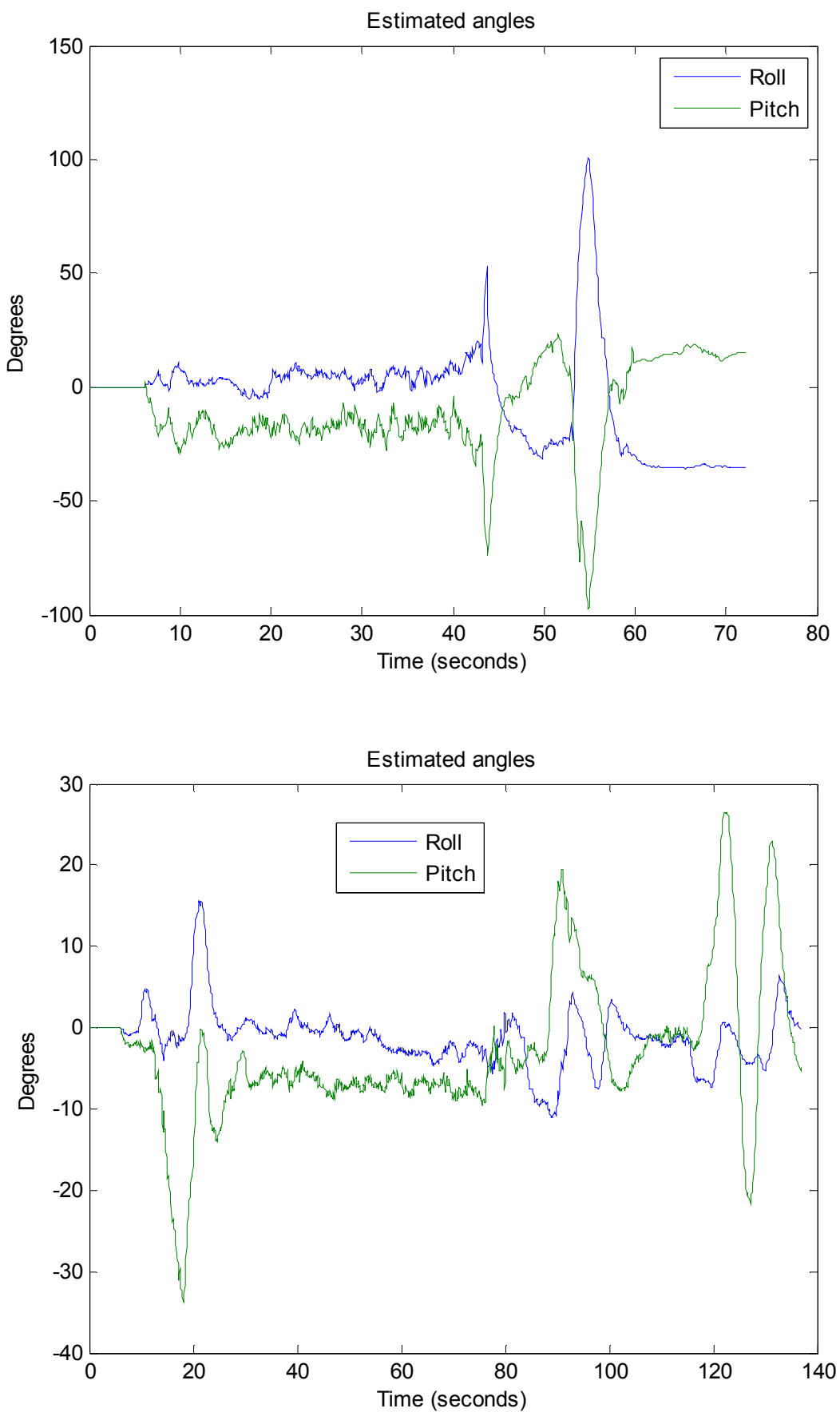

Figure 46: Pitch and Roll Variations during Tip

Detection in Frames 3 and 4 for Subject \#4 
Figures 47 and 48 present 4 graphs of the estimated angles within frames when a tip event was detected for subject \#17.
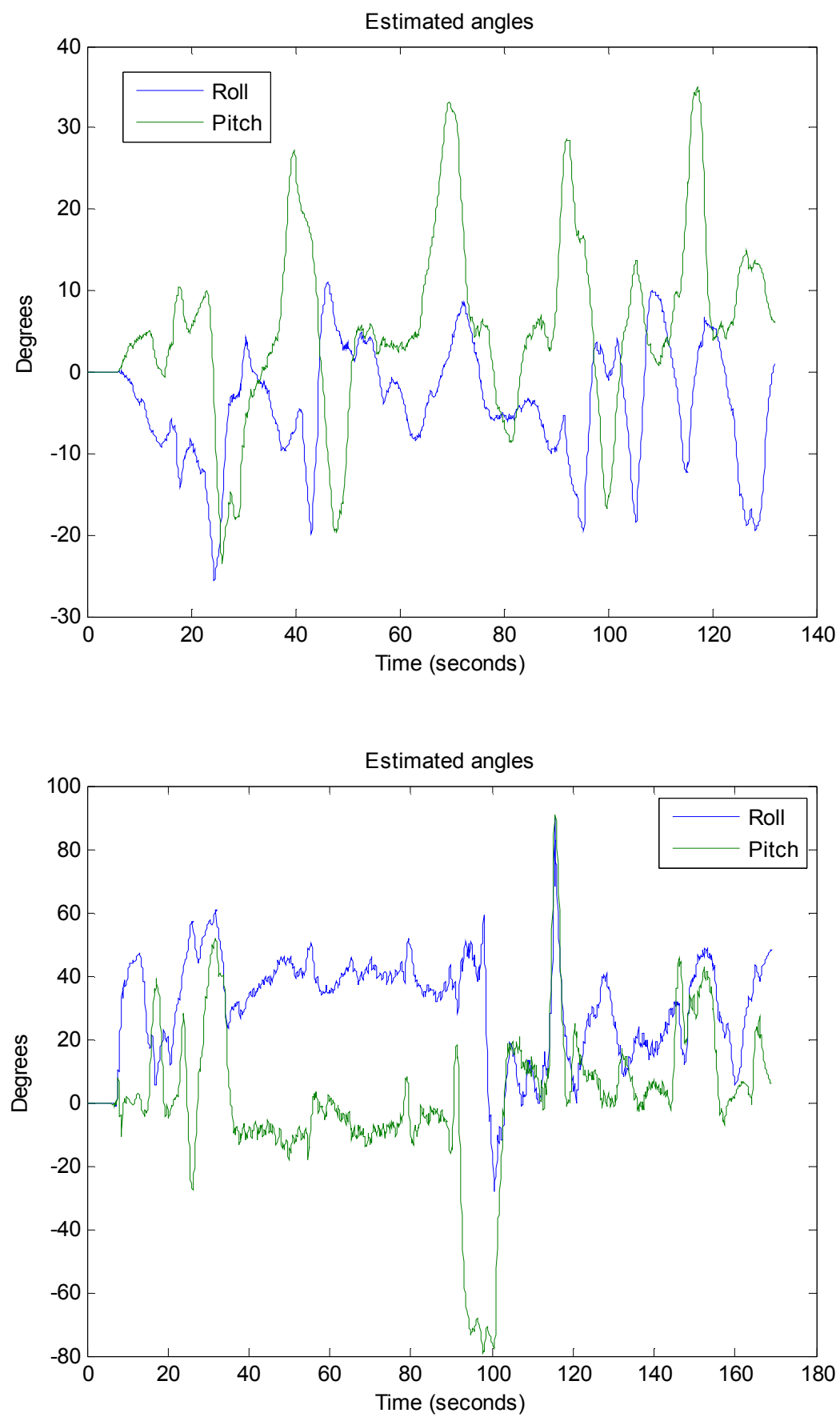

Figure 47: Estimated Angles in Frames 1 and 2 within which Tips were Detected for Subject \#17 

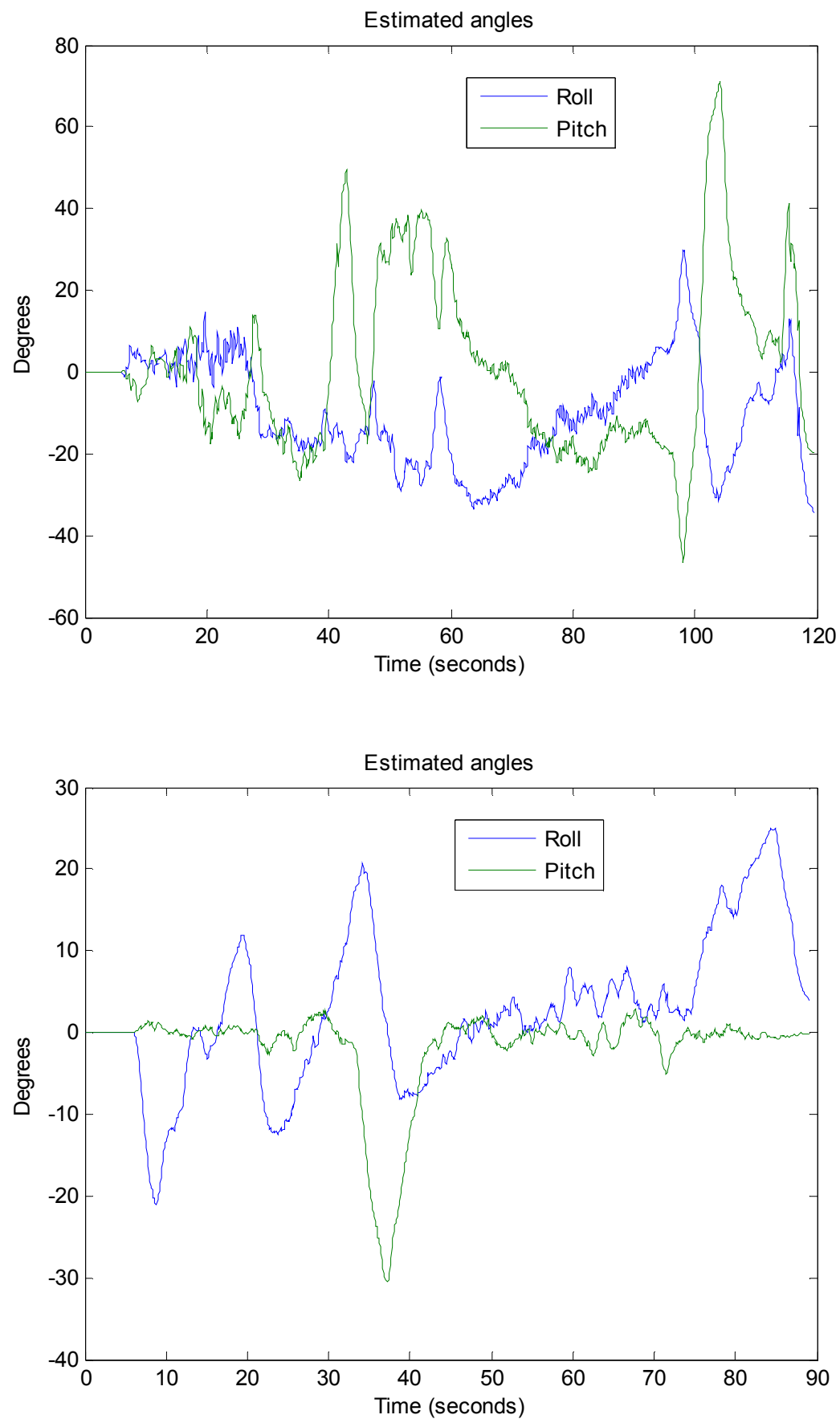

Figure 48: Estimated Angles in Frames 3 and 4 within which Tips were Detected for Subject \#17 
Figures 49 and 50 graphically display the estimated angles for frames in which a tip was detected for subject \#8.
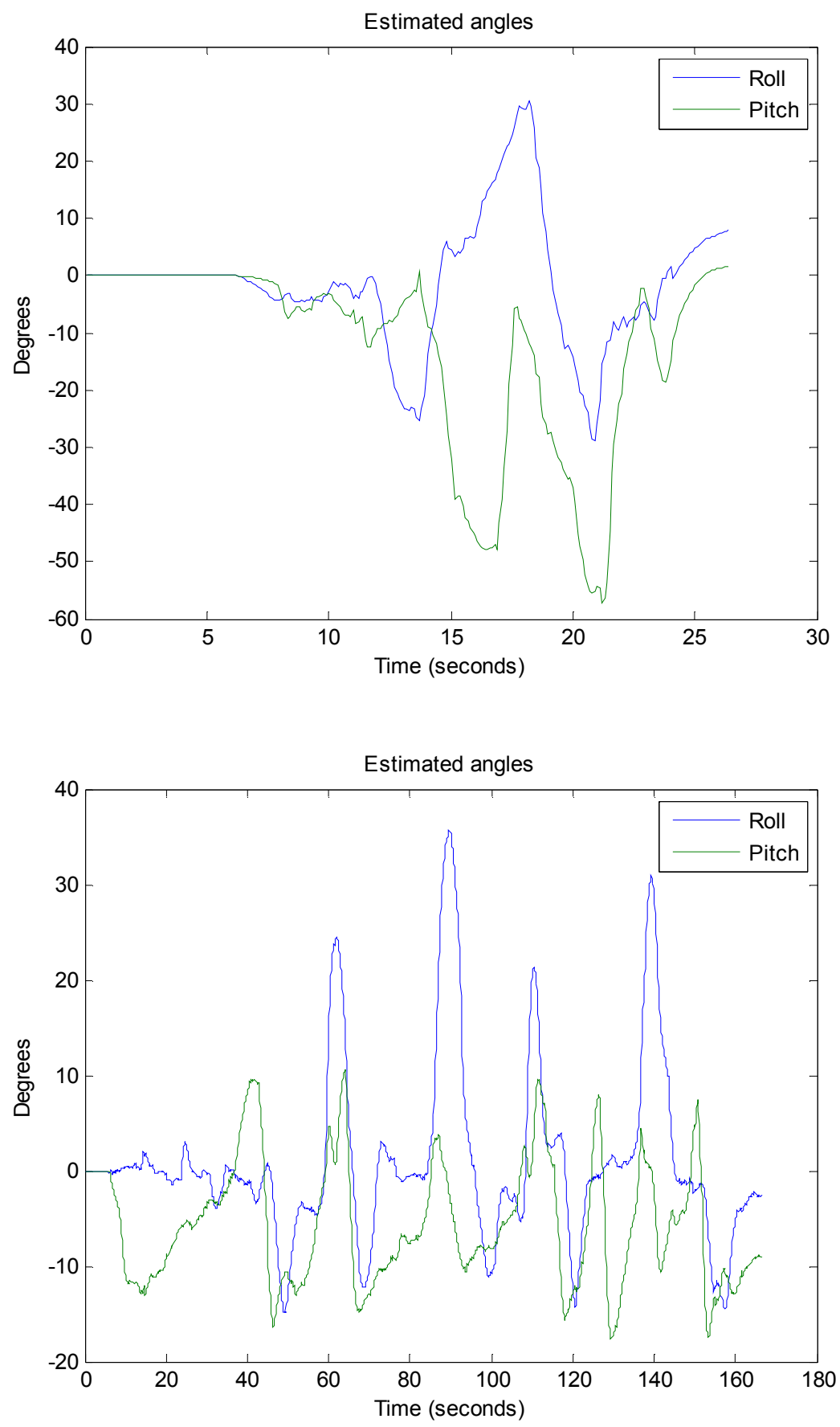

Figure 49: Pitch and Roll Angles during Frames 1 and 2 within which Tips were Detected for Subject \#8 

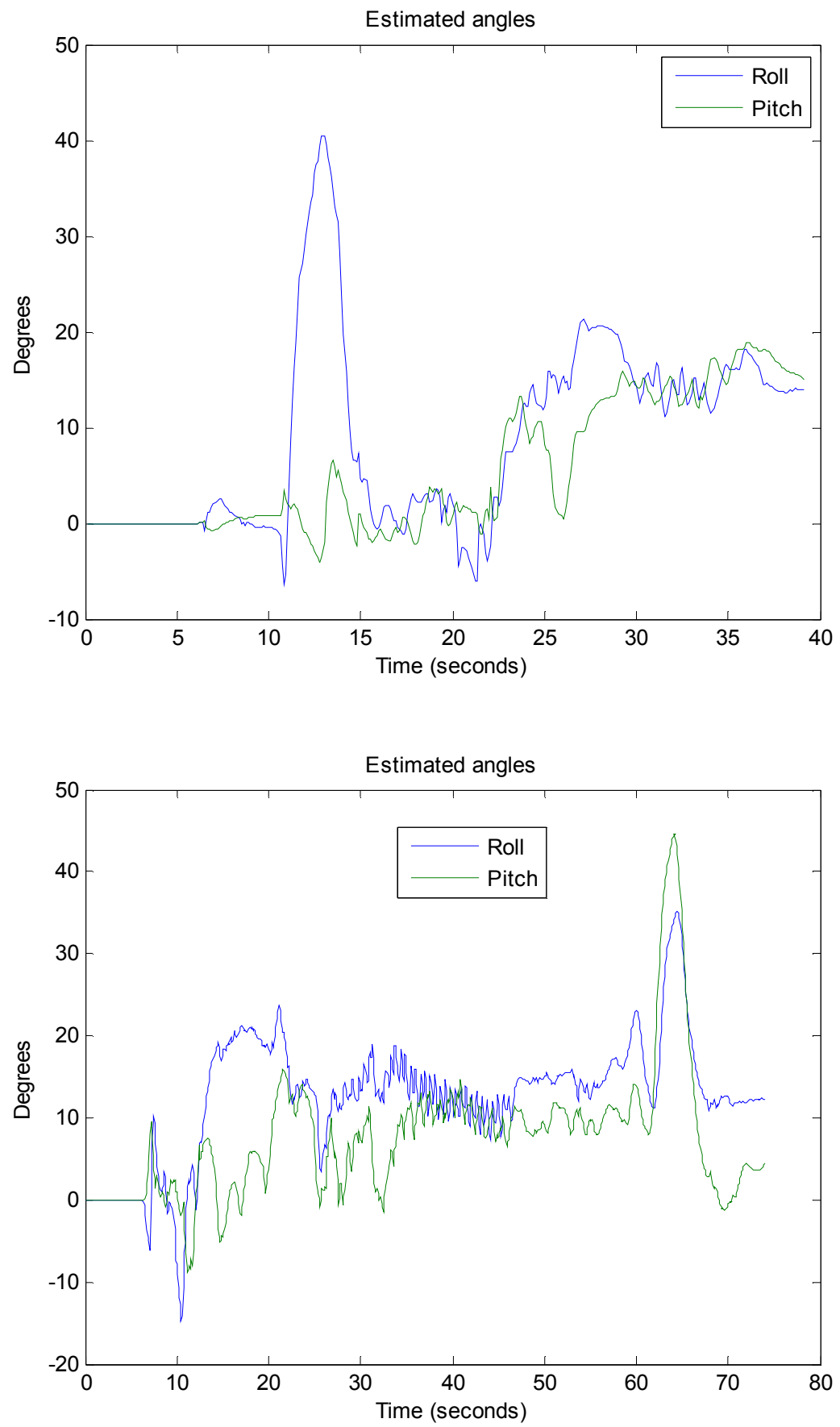

Figure 50: Pitch and Roll Angles during Frames 3 and 4 within which Tips were Detected for Subject \#8 
Figure 51 graphically displays the pitch and roll angles for frames during which fall events were detected for subject \#4.
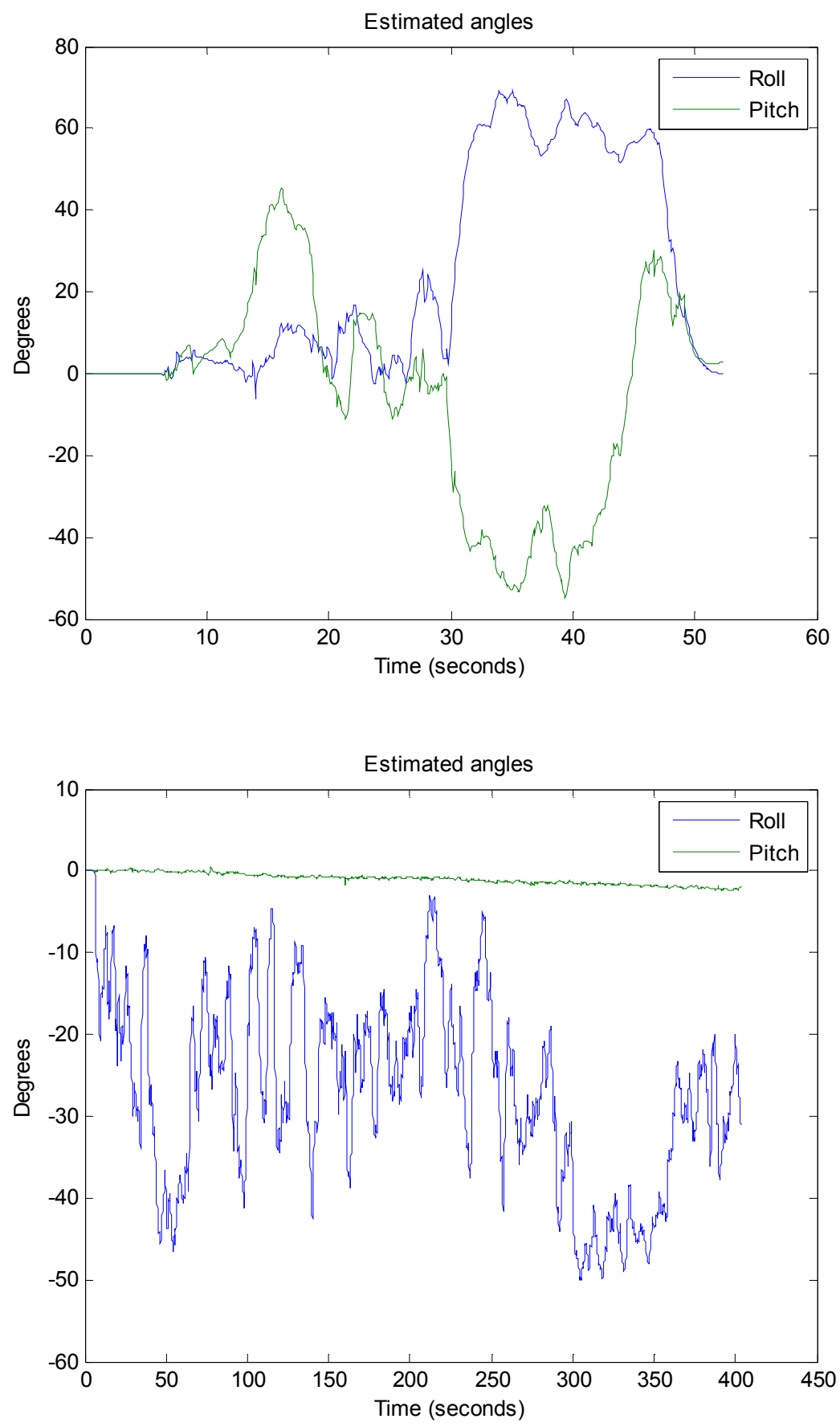

Figure 51: Pitch and Roll Angles during Frames within which Falls were detected for subject \#4 
Figure 52 graphically displays the pitch and roll angles on frames during which falls events were detected for subject \#17.
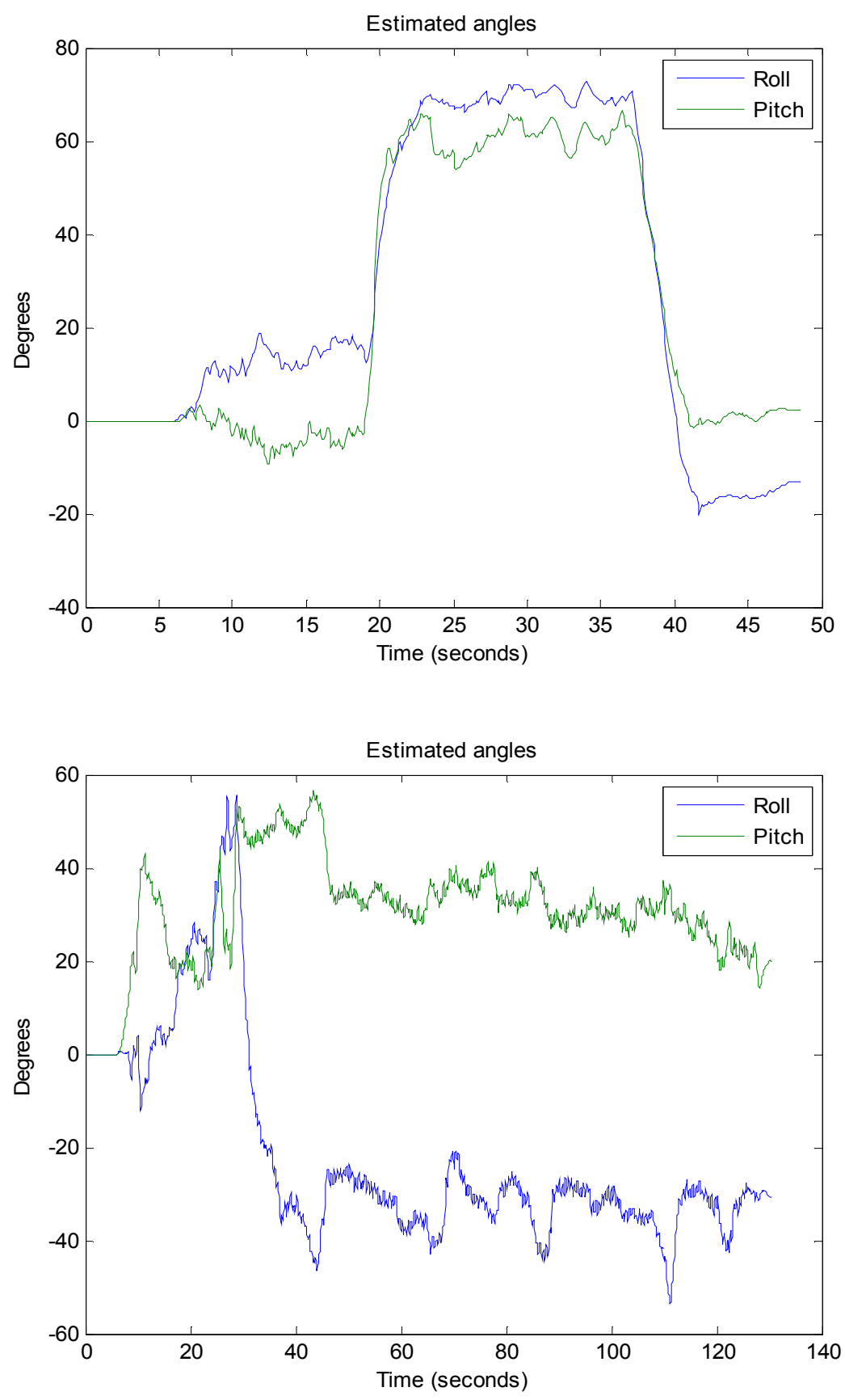

Figure 52: Pitch and Roll Angles during Frames within which Falls were Detected for Subject \#17 


\section{Chapter 7}

\section{Conclusions and Future Work}

\subsection{Conclusions}

A motion-logger was successfully developed to support the study of tips and falls of wheelchair users. A logging and estimation system provided accurate information about the motion and attitude of the wheelchair for successfully determining risky situations.

An embedded system was built for data acquisition from wheelchairs. The system was designed to collect data for over long periods of time, which could be several months. The data, from sensors containing relevant information about the motion of the wheelchair, was stored in an SD memory card. Data was also obtained and stored for battery condition and temperature. The date and time at which the data was recorded was included for further analysis.

A Kalman filter based solution was implemented for pitch and roll angles estimation after collecting information from a five degrees of freedom IMU. An algorithm for detecting tips and falls was developed using the estimated angles in order to report those events, as well as the dates and times on which they occurred. The device 
was properly tested and installed on users' wheelchairs during short and long-term data acquisition.

When the wheelchair data is combined with demographic records the solution provides important information regarding the nature of risks encountered by wheelchair users. In addition, the solution provides for the investigation of the nature, causes, consequences and costs of wheelchair users falls.

\subsection{Future Work}

Several functionalities have been identified as possible future development areas for making the system more robust and user friendly. These functionalities are:

- Include a wireless interface for downloading the logged in data from the device to the PC.

- Integrate with a mobile device so that transmission over the cellular network can be accomplished. Therefore, users would not be required to interact with any storing device during the research since the information would be directly received in a specific server.

- Design specific hardware for the proposed solution. This would provide an independent power supply for the Real Time Clock module, which would eliminate the problem of losing the real time information for the time-stamps reported for tips and falls events. 
- Evaluate the possibility of implementing the Kalman filter based angles estimation algorithm and the tips and falls detection software in the embedded device instead of in an external computer. 


\section{References}

[1] ClinicalTrials, "Epidemiology and Cost of Falls in Veterans with a Spinal Cord Injury", clinicaltrials.gov, [Online], Available:

http://clinicaltrials.gov/ct2/show/N CT00119522?term=fall+wheelchair\&rank=2, [Accessed August 13, 2008]

[2] ClinicalTrials, "Characterizing Wheelchair-Related Falls in Elderly Veterans", clinicaltrials.gov, [Online], Available: http://clinicaltrials.gov/show/NCT003632466, [Accessed January 19, 2008]

[3] D. Ding, E. Leister, R.A. Cooper, D. Spaeth, R. Cooper, A. Kelleher and M.L. Boninger, "A Wheelchair Usage Monitoring/Logging System”, Proceedings of the 2005 IEEE Engineering in Medicine and Biology $27^{\text {th }}$ Annual Conference, pp 6897-6899

[4] D. Ding, R.A. Cooper, R. Cooper, and A. Kelleher, "Monitoring Seat Feature Usage among Wheelchair Users", Proceedings of the $29^{\text {th }}$ Annual International Conference of the IEEE EMBS, pp. 4364-4367, 2007

[5] J. A. Hesch and S. I. Roumeliotis, “An Indoor Localization Aid for the Visually Impaired", 2007 IEEE International Conference on Robotics and Automation, pp 3545-3551

[6] Onset Computer Corporation, "HOBO Pendant G Acceleration Data Logger", onsetcomp.com, [Online],

Available: http://www.onsetcomp.com/products/data-loggers/ua-004-64?pid=474, [Accessed August 12, 2008]

[7] ARATA National Conference, "The "Black Box: A powered wheelchair usage monitor", www.ebility.com/arata/, [Online], Available: http://www.ebility.com/arataconf06/abstracts/poster_wms_kolb.html, [Accessed August 13, 2008] 
[8] SparkFun, "Logomatic V1.0 Universal Data Logging Device", sparkfun.com, [Online], Available:

http://www.sparkfun.com/datasheets/Widgets/Logomatic/Logomatic_V1_0.PDF, [Accessed January 20, 2008]

[9] SparkFun, "MEMS Accelerometer ADXL330", sparkfun.com, [Online], Available: http://www.sparkfun.com/datasheets/,

Components/ADXL330_0.pdf., [Accessed January 20, 2008]

[10] SparkFun, "Integrated Dual-Axis Gyro IDG-300”, sparkfun.com, [Online], Available:

http://www.sparkfun.com/datasheets/Components/IDG-300_Datasheet.pdf, [Accessed January 20, 2008]

[11] SparkFun, "Polymer Lithium Ion Batteries", sparkfun.com, [Online], Available: http://www.sparkfun.com/datasheets/Batteries/UnionBattery-2000mAh.pdf, [Accessed January 20, 2008]

[12] SparkFun, "LiPo Charger", sparkfun.com, [Online]. Available: http://www.sparkfun.com/datasheets/BreakoutBoards/max1555_breakout.PDF, [Accessed January 20, 2008]

[13] D. Simon, "Optimal State Estimation: Kalman, Hœ, and Nonlinear Approaches", Wiley-Interscience, 2006

[14] University of North Carolina, "An Introduction to the Kalman Filter", cs.unc.edu, [Online], Available: http://www.cs.unc.edu/ welch/kalman/kalmanIntro.html, [Accessed September 1, 2008]

[15] P. Zarchan and H. Musoff, "Fundamentals of Kalman Filtering: A Practical Approach", American Institute of Aeronautics and Astronautics, 2000 
Appendices 


\section{Appendix A: Tilt Sensor Instructions}

There is a significant problem with subjects who use wheelchairs or scooters and are experiencing tips and/or falls. This tilt sensor will be placed on the wheelchair in order to monitor the number of tips and/or falls experienced over a period of time.

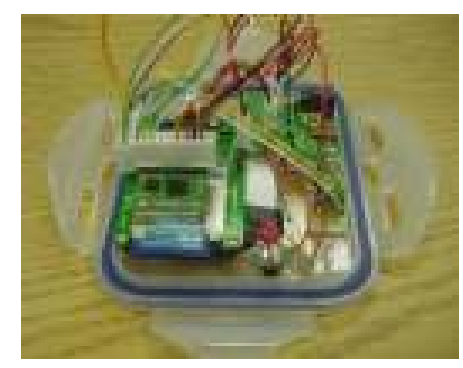

Inside the sealed Tilt Sensor case attached to your power wheelchair or scooter there is a small lithium ion rechargeable battery and a removable memory card. The battery will need to be recharged every day(see instructions). The memory card will need to be removed and replaced every month and mailed back to Bob Byrd at James A Haley VA Hospital Subject Safety Center, 11605 N Nebraska Avenue Tampa, Florida 33612-5738 using the self-addressed, stamped envelope that will be given to you, (see instructions).

Eligibility requirement for subjects:

- A minimum of 62 years old,

- Have a history of using a wheelchair for at least 3 months,

- Plan to use a wheelchair for an extended period of time,

- Have a caregiver present who can assist with the Tilt Sensor. 


\section{Appendix A: (Continued)}

Instructions on How to Charge the Battery:

- Open the clear plastic container by pulling down on the 4 tabs that holds the cover on the sensor case,

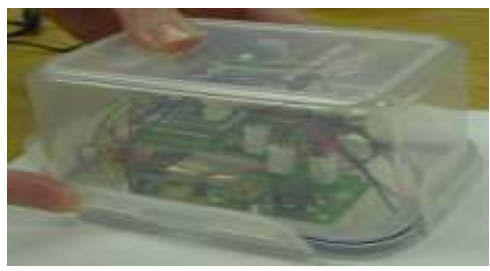

- Gently remove the cover,

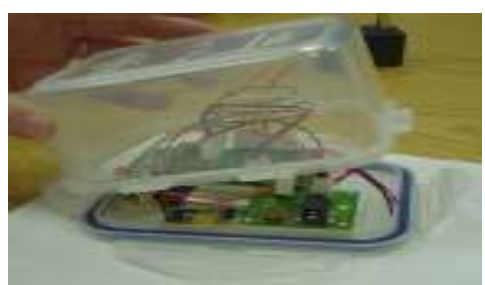

- Connect the external power supply to the male connector located on the sensor board,

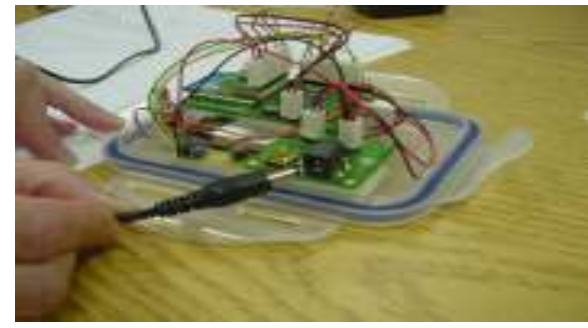

“DO NOT PUSH THE RED PUSHBUTTON SWITCH - THIS ACTION WILL RESET THE TIME REFERENCE OF THE SYSTEM - IF BY MISTAKE THE TIME WAS RESET PLEASE (RECORD Date and TIME)”. Call the Research Team at 1-866-406-9954 for any assistance. 


\section{Appendix A: (Continued)}

- Connect the wall adapter to the power outlet and charge the battery for at least 8 continuous hours.

- After the battery has been charged, remove the adaptor and seal the sensor case by replacing the cover and locking it with the 4 tabs.

Memory Card Handling:

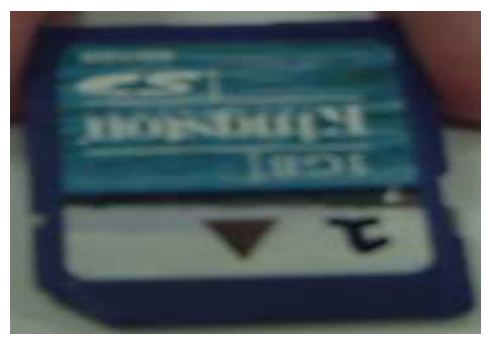

- The memory card must be removed and replaced with a new memory card once each month.

- A call will be made to subjects each month as a reminder to remove and replace the memory card.

- After removal, the memory card is to be mailed in the self-addressed, stamped envelope to:

Bob Byrd James A Haley VA Hospital Subject Safety Center 11605 N Nebraska Ave

Tampa, Florida 33612-5738. 


\section{Appendix A: (Continued)}

Instructions on How to Remove and Replace the Memory Card:

- Open the clear plastic container by pulling down on the 4 tabs, which hold the cover of the sensor case,

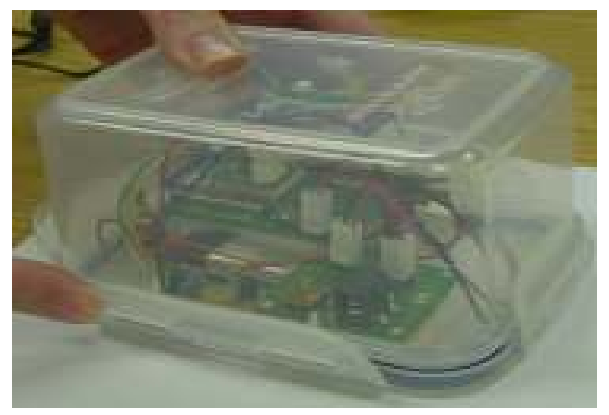

- Gently remove the cover,

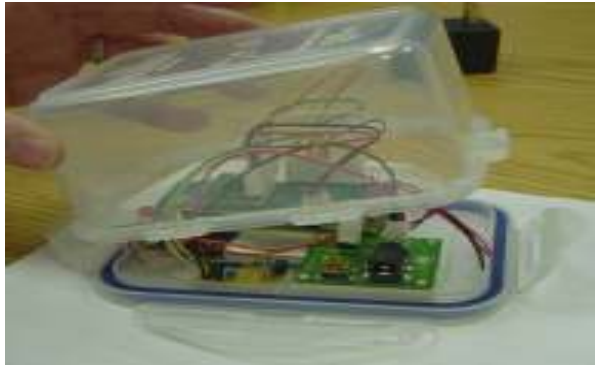

- Gently PUSH IN the memory card to release the holding mechanism,

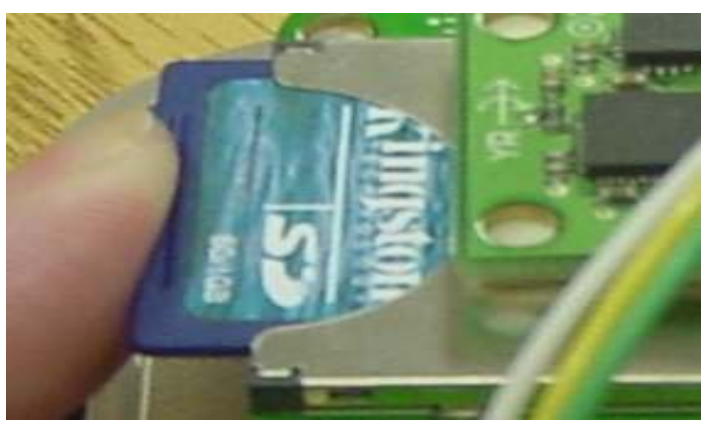




\section{Appendix A: (Continued)}

- Smoothly PULL OUT the memory card,

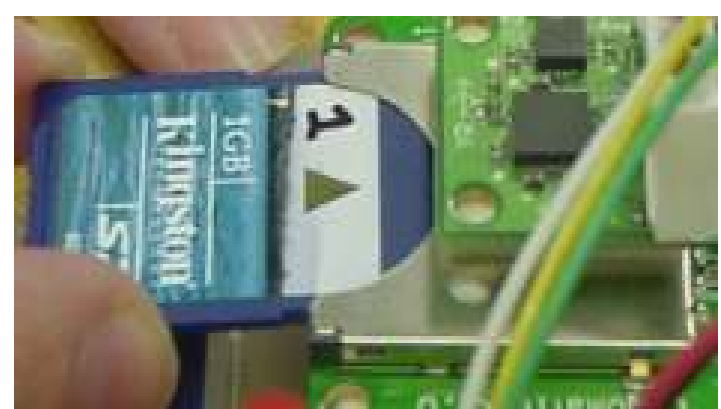

- Replace the removed memory card with a new memory card. Make sure the card identification number is facing up following the arrow direction inscribed on the card,

- RESET THE SYSTEM BY PUSHING THE RED PUSH BUTTON SWITCH AT LEAST ONCE. PUSH UNTIL THE RED PUSH BUTTON SNAPS INTO PLACE,

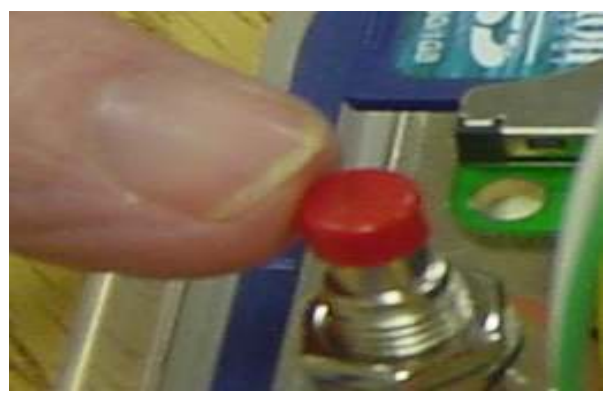

- Place the old memory card in the self-addressed, stamped envelope and seal it,

- Seal the sensor case by placing the cover back and locking it with the 4 tabs,

- Mail the memory card to:

Bob Byrd

James A Haley VA Hospital Subject Safety Center

11605 N Nebraska Avenue

Tampa, Florida 33612-5738

Thank you for agreeing to participate in our efforts to reduce wheelchair tips and falls and keep our veterans safe. 